\title{
Volume 7, Number 3
}

\section{Hipatia Press}

www.hipatiapress.com

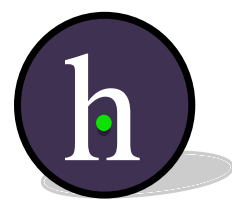

Quantum Masculinities: Doing Gender with Max Tegmark's Mathematical Universe Hypothesis - Joseph Gelfer \& Joseph Organ.

Comunicación de Marca en Instagram, ¿Una Cuestión de Género? El Rol del Influencer de Moda - Raquel Martínez-Sanz \& Cristina Gonzalez Fernández.

\section{Articles}

Production of Gender: A Study on Performativity in Female-To-Male Transsexuals - Tanupriya \& Dhishna Pannikot

Bidirectional Violence among Male and Female University Students: Comparison of Observations and Results between Two Countries? Teresa Fernández de Juan \& Lourdes Florez Madan

The Social Life of Gender - Vladia lonescu. 


\section{Hipatia Press \\ www.hipatiapress.com}

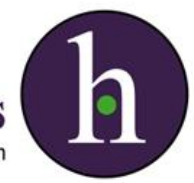

Instructions for authors, subscriptions and further details:

\section{http://mcs.hipatiapress.com}

\section{Quantum Masculinities: Doing Gender with Max Tegmark's Mathematical Universe Hypothesis}

Joseph Gelfer ${ }^{1} \&$ Joseph Organ²

1) Université Catholique de l'Ouest, France

2) Moscow Institute of Physics and Technology, Russia

Date of publication: October $21^{\text {st }}, 2018$

Edition period: October 2018 - June 2019

To cite this article: Gelfer, J. \& Organ, J. (2018). Quantum Masculinities: Doing Gender with Max Tegmark's Mathematical Universe Hypothesis.

Masculinities and Social Change,7(3), 213- 229. doi:

10.17583/MCS.2018.3470

To link this article: http://doi.org/10.17583/MCS.2018.3470

\section{PLEASE SCROLL DOWN FOR ARTICLE}

The terms and conditions of use are related to the Open Journal System and to Creative Commons Attribution License (CC-BY). 


\title{
Quantum Masculinities: Doing Gender with Max Tegmark's Mathematical Universe Hypothesis
}

Joseph Gelfer

Université Catholique de l'Ouest

Joseph Organ

Moscow Institute of Physics \& Technology

\begin{abstract}
In his book Our Mathematical Universe, Max Tegmark proposes a way of viewing "reality" as a multiverse of parallel universes governed by mathematics. In this article we take a few of Tegmark's more accessible ideas and combine them with the study of masculinities to form "quantum masculinities." Specifically, we use Tegmark's presentation of the multiverse and the quantum state of superposition as a thinking tool for imagining not just multiple masculinities but infinite and contradictory masculinities. We then mobilize this newly proposed concept of quantum masculinities in two contexts. First, we put quantum masculinities in dialogue with Judith Butler's theory of gender performativity to question to what degree gender performance requires an observer. Second, we explore how far quantum masculinities are reconcilable with The Five Stages of Masculinity. We conclude with some discussion about the categories of "I" and "we" in imagining the self, as well as how the study of masculinities might evolve.
\end{abstract}

Keywords: masculinities, quantum physics, multiverse, gender performativity, superposition, parallel universes 


\section{Masculinidades Cuánticas: Haciendo Género con la Hipótesis del Universo Matemático de Max Tegmark}

Joseph Gelfer

Université Catholique de l'Ouest

Joseph Organ

Moscow Institute of Physics \& Technology

\section{Resumen}

En su libro Our Mathematical Universe, Max Tegmark propone una forma de ver la "realidad" como un multiverso de universos paralelos gobernados por las matemáticas. En este artículo tomamos algunas de las ideas más accesibles de Tegmark y las combinamos con el estudio de las masculinidades para formar las "masculinidades cuánticas". Específicamente, utilizamos la presentación de Tegmark del multiverso y el estado cuántico de superposición como una herramienta de pensamiento para imaginar, no solo masculinidades múltiples, sino que masculinidades infinitas y contradictorias. Después vamos más allá y planteamos este nuevo concepto de masculinidades cuánticas en dos contextos. En primer lugar, ponemos en contacto las masculinidades cuánticas con la teoría de la performatividad de género de Judith Butler para preguntarnos en qué medida el desempeño de género requiere una persona observadora. En segundo lugar, exploramos hasta qué punto las masculinidades cuánticas son reconciliables con Las Cinco Etapas de la Masculinidad. Concluimos con una discusión sobre las categorías de "yo" y "nosotros" al imaginarnos a nosotros mismos, y sobre cómo podría evolucionar el estudio de las masculinidades.

Palabras clave: masculinidades, física cuántica, multiverso, performatividad de género, superposición, universos paralelos 


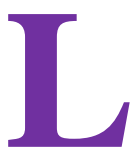

ike many things in the contemporary world, it started with a Tweet. On Twitter, one of us had noted that "masculinity in 2016 appears to be in simultaneous states of extremity: increased normativity, increased queerness" (@ drjosephgelfer, 2016b), to which the anthropologist Dick Powis replied, "Dibs on "quantum masculinities' \#buzzwords \#jobmarket”(@dtpowis, 2016). Some phrases demand to be unpacked and "quantum masculinities" is one of them, even if that means reverse-engineering meaning that was not originally intended.

An orthodox treatment of quantum masculinities would no doubt investigate masculine performances within the field of quantum physics, and indeed we can find studies that do just that in comparable physics communities (albeit minus the phrase "quantum masculinities"). Helena Pettersson (2011) provides an ethnographic study of plasma physicists in the United States, noting that the subject is a primarily male domain, how the lab in which she did her study had an atmosphere of "boys and their toys" ( $p$. 55 ), homosociality, manual labor, and the dangerous and dirty nature of the work, all of which signify normative masculinity. Allison J. Gonsalves, Anna Danielsson and Helena Pettersson (2016) offer three case studies in Sweden, Canada and the United States within a predominantly learning environment across various physics subfields such as astrophysics, theoretical high energy particle physics, and solid state physics. In these case studies, masculine ideals were again presented as the norm, to the point where even female participants "reject traditional femininity, and may be seen as performing a type of female masculinity instead" (p. 12). No doubt similar findings would be discovered were such a study made of the specific subfield of quantum physics, adding to what is now a long history of feminist literature regarding the gendered nature of science (for example, Keller, 1985; Harding, 1986; Schiebinger, 1999), including quantum physics (Barad, 1995). In such a context, quantum masculinities is like a game of "masculinities spotting" in which one keeps a tally of the number of people who perform normative masculinity (including, as we see above, women), and those who perform (willingly or unwillingly) alternative forms of masculinity.

However, this article is not an orthodox treatment of quantum masculinities; it takes a more literal approach, posing some thought experiments about masculinities that can be had by engaging theories of the quantum and mathematical universe as proposed by Max Tegmark (2014). 
This article is not part of some greater project, rather the documentation of some thoughts that have arisen through a critical reading of two texts that were never intended to be put in dialogue. The first section introduces Tegmark's concept of the "multiverse" and a context in which we can imagine not just multiple masculinities but infinite masculinities. The second section then debates Tegmark's presentation of the quantum state of superposition, which again opens up a space not just for multiple masculinities but simultaneously contradictory masculinities. This section also puts Tegmark in conversation with Judith Butler's concept of gender performance (Butler, 1999), questioning whether a performance needs an observer, and if so, whether this closes down the possibility of masculinities in superposition. Of course, there are - to say the least-significant epistemological and ontological differences between the approaches of Tegmark and Butler: our aim is not to reconcile these differences (or even to explore them), rather to see what possibilities surface when we hold these two thinkers in productive tension. The penultimate section explores to what extent quantum masculinities are reconcilable with The Five Stages of Masculinity (Gelfer, 2016), bringing the two together to question the notion of the "I" in perceptions of the self. In conclusion, we explore some implications of the "I" and "we" in the multiverse and look at under what circumstances quantum masculinities are distinguishable from quantum femininities. We finish by locating this discussion in the context of the study of masculinities and how this might usefully evolve.

Some of these thought experiments are not entirely serious, and none of them are scientific. We are mindful that the subject of this article — using the terms "quantum" and "masculinities"- sounds an awful lot like a joke combining two infamous hoax articles, Transgressing the Boundaries: Towards a Transformative Hermeneutics of Quantum Gravity (Sokal, 1996) and The Conceptual Penis as a Social Construct (Boghossian \& Lindsay, 2017). But there is a point - that no doubt shifts from reader to readerwhere tongue-in-cheek comments take a serious turn, where the playfulness opens up a different line of thought that might otherwise have remained closed: we will explore this boundary in the conclusion.

This article also seeks to play its part in redressing an imbalance in the study of masculinities, which is typically characterized by a modernist approach that has made the subject look a lot like sociology (Beasley, 2013, 
2015). This is in comparison to other fields of gender studies such as feminist theory and queer theory that have complemented such modernist approaches with more postmodern and philosophical approaches. There are of course many masculinities researchers who use a postmodern approach, and a more philosophical worldview can also be found in the largely - and unfairlyignored field of Critical Men's Studies in Religion (Krondorfer, 2017), but the study of masculinities nevertheless needs to be fleshed out. Lucas Gottzén (2018) has affirmed 'that we need both 'modern' and 'postmodern' approaches" (p. 85) to masculinities. This is of course commendable, but it also feels like a somewhat outdated request, like dragging the study of masculinities into the early 1990s (undoubtedly a sweet spot for gender studies, when feminism had reached new levels of maturity, queer theory was firing on all cylinders, and what are still the most important theories in the study of masculinities had been set down). Why ask to play catch-up when you can play leap frog? Why not think bigger? Why not engage with even ostensibly unrelated lines of questioning and worldviews - such as quantum mechanics - to see if new thoughts are revealed?

\section{The Multiverse}

Tegmark is a Professor of Physics at Massachusetts Institute of Technology. His book Our Mathematical Universe: My Quest for the Ultimate Nature of Reality (2014) can be described as a "popular science" book, which means non-scientists can understand it. He employs what he describes as a "Dr. Jekyll/Mr. Hyde Strategy" (p. 244) in which Jekyll provides the traditional physics and Hyde the speculative physics. His work is not without its critics from physicists and mathematicians who have no time for such crank-like alter-egos (Frenkel, 2014; Woit, 2014). In this article we have no interest in questioning Tegmark's scientific rigor. The objective is to take a few of his ideas and use them as a jumping-off point for thinking around masculinities.

At the heart of Tegmark's book is the idea of the multiverse, which comprises four levels. The Level I multiverse comprises an infinite number of parallel universes - based on the assumption that space is infinite - which are "distant regions of space that are currently but not forever unobservable; they have the same effective laws of physics but may have different histories" (p. 139). The Level II multiverse comprises parallel universes that are 


\section{Gelfer \& Organ - Quantum Masculinities}

"distant regions of space that are forever unobservable because space between here and there keeps inflating; they obey the same fundamental laws of physics, but their effective laws of physics may differ" (p. 139).

The Level III and IV multiverses are beyond the scope of this article. However there are other clear lines of exploration for the mathematically brave. The Level III multiverse operates at a quantum level in the multidimensional Hilbert space, which is a purely mathematical domain. This type of multi-dimensional space feels very intuitive when discussing the multiplicity of masculinities. Indeed, looking for a more philosophical analogy for Hilbert space one could do worse than look at the Deleuzian concept of rhizomatic or "smooth" space (Deleuze \& Guattari, 1987, p. 12), which we have previously proposed as a space for deregulated gender performances (Gelfer, 2009, pp. 168-169). More recently, Deleuze has been discussed in the context of Hilbert space (Christiaens, 2014), and more generally in quantum mechanics (Crockett, 2013, pp. 148-162).

Given that space is infinite, Tegmark suggests anything that could exist in these infinite parallel universes does exist, including other versions of us. Remember, the Level I multiverse has the same laws of physics but different histories. This means that the multiverse "contains many more people who are almost like you, yet slightly different" but also, given its infinite capacity, "out of all your infinitely many look-alikes out there on other planets, there's also one who speaks English, lives on a planet identical to Earth, and has experienced a life completely indistinguishable from yours in all ways. This person subjectively feels exactly like you feel" (p. 123).

In the study of masculinities, "multiple masculinities" has a reasonably limited meaning. Harry Brod (1987) refers to "the study of masculinities and male experiences as specific and varying social-historical-cultural formations" (p. 2). Multiple masculinities have also been applied to females (Butler, 1999; Halberstam, 1998). In a more individualized and internalized context, concepts of the "multiple self-aspects framework" (McConnell, 2011) and the "dialogical self" (Hermans \& Kempen, 1993) have been applied to multiple masculinities within the individual (Gelfer, 2012).

In the Level I multiverse, multiple masculinities take on an altogether different meaning. We move from identifying different masculine performances in our common understanding of space and time (here a masculine performance in a Bangladeshi market, there a masculine 
performance in a play by Shakespeare), beyond different hypothetical performances (masculinity can mean anything you want it to mean), right through to the concurrent reality of all possible masculine performances. Multiple masculinities are not, then, simply a project that seeks to trouble how gender is regulated in society on Earth; rather, infinite masculinities are a necessity that is required to approximate the "reality" of the many versions of us that exist in the Level I multiverse (whether near-identical copies, or copies that have significantly diverged due to the different unfolding of local histories).

In the Level II multiverse there are further possibilities. Tegmark opens a section discussing Level II with the single exclaimed word "Diversity!" ( $p$. 134). Remember, Level II occupies distant areas of space that "obey the same fundamental laws of physics, but their effective laws of physics may differ." Tegmark proposes that "fundamental laws of physics, which by definition hold anywhere and anytime, can give rise to a complicated physical state of affairs where the effective laws of physics inferred by self-aware observers vary from place to place" (p. 134, original emphasis).

Of course, "diversity" possesses an almost magical resonance in the study of masculinities (and, more generally, gender and sexuality). The different effective laws of physics provided at Level II open up various possibilities of different physical formations to combine with masculinities. At Level I we "merely" have a different set of histories that demand an infinite set of masculine social constructions. At Level II the complex intertwining of sex and gender can take on-must take on, given its literally infinite varietyhitherto unimagined permutations. There is a further metaphorical application here to multiple masculinities. The "fundamental laws of physics, which by definition hold anywhere and anytime" are analogous to hegemonic masculinity whereas the "effective laws of physics inferred by self-aware observers vary from place to place" are analogous to subordinate or resistant masculinities.

\section{Superposition}

Tegmark quips, "If you want to stir up a cocktail party by sounding like a quantum physicist, another buzzword you'll need to drop is superposition: a particle that's both here and there at once is said to be in a superposition of here and there" (p. 176). So let's drop it in. 
The gateway most people have to superposition is the example of Schrödinger's cat. In this thought experiment the physicist Erwin Schrödinger places a cat in a chamber with a small amount of radioactive substance that may or may not kill the cat within an hour due to the radioactive substance decaying. If we were to run this experiment multiple times, half the time the cat would be alive when we open the box, half the time dead. But importantly here, it is our observation of the event that gives it a conclusion: before we open the box, the cat is in a superposition of being both dead and alive. Tegmark describes superposition as a "quantummechanical situation where something is in more than one state at once, for example in two different places" (p. 179).

Superposition offers us another analogy for multiple masculinities. In other words, masculinity can be both this and that at the same time. In a basic sense we might say that masculinity is in a superposition when it can be either hegemonic or subordinate (or any number of other individual masculine performances). In a more nuanced sense we might say that masculinity is both hegemonic and subordinate (or any number of other individual masculine performances) at the same time: this is like a quantum version of "hybrid masculinities" (Bridges \& Pascoe, 2014) where men selectively incorporate subordinate into hegemonic masculine performances. Superposition also seems to have a striking resemblance to Judith Butler's understanding of gender performativity, where "genders can be neither true nor false, neither real nor apparent, neither original nor derived" (p. 180); or at least a re-casting of this statement where "genders can be either true or false, either real or apparent, either original or derived" or, even better, "genders can be both true and false, real and apparent, original and derived."

But the analogy of superposition for multiple masculine performances does not extend very far. Let's go back and explore Butler's gender performativity a bit further in the context of Schrödinger's cat. The cat is in a superposition until it is observed: it is both alive and dead; both this and that; it has multiplicity. However, Butler implies that performativity requires observation: while our gendered "reality is fabricated as an interior essence" it is also the case that this "interiority is an effect and function of a decidedly public and social discourse, the public regulation of fantasy through the surface politics of the body" (p. 173). It is this public discourse and regulation that implies observation, and thus the collapsing of the superposition. 
While Butler is ultimately concerned with the politics of the body, Tegmark is more concerned with the physical properties of the body (or object). Tegmark states that "an object can only be found in two places at once in a quantum superposition as long as its position is kept secret from the rest of the world. If the secret gets out, all quantum superposition effects become unobservable" (p. 199). Butler implies that gender performance requires public discourse. It would seem, then, that we have reached an impasse if we try and extend superposition beyond a metaphor for multiple masculinities: we can only have gender performances in superposition if they take place in secret, but we cannot do gender performances in secret. Indeed, Tegmark suggests that something in a state of superposition does not even require a conscious observer to collapse the state, merely the transfer of information, such as an object being struck by light and air (pp. 199-200). In this sense, even if Judith Butler was the last person left not just on Earth, but in the whole multiverse, her gender performance could not be kept secret unless she locked herself inside a lightless perfect vacuum; and then the performance would not last very long because she would suffocate. So much for superposition.

\section{Quantum Masculinities and The Five Stages of Masculinity}

The Five Stages of Masculinity categorizes various philosophical, ideological and political worldviews regarding masculinity (Gelfer, 2016). Stage 1 refers to "unconscious masculinity" where people adhere to ideals of normative masculinity without any awareness of its existence. Stage 2 refers to "conscious masculinity" where people consciously adhere to ideals of normative masculinity. Stage 3 refers to "critical masculinities" where people provide a systemic critique of normative masculinity. Stage 4 refers to "multiple masculinities" where people reject normative masculinity and perform any number of masculinities (including ones that appear normative, but minus the regulation implicit in normativity). Stage 5 refers to "beyond masculinities" where people reject the idea of masculinity.

At this point, we have suggested that "quantum masculinities" as a term combines two key ideas. First, masculinities are infinite given the infinite nature of the multiverse, and that those masculinities may follow different histories that share our same laws of physics (Level I multiverse) or may 
follow different histories and take on different localized laws of physics (Level II multiverse). Second, superposition functions as an interesting analogy (albeit not extendable very far) for explaining why masculinities can simultaneously be in different and/or contradictory states.

We now want to show how quantum masculinities and The Five Stages of Masculinity are reconcilable. At Stage 4, "each individual dwells in a category of sex and gender as unique as their fingerprint" (Gelfer, 2016, p. 279). In essence, at Stage 4, masculinities are infinite as "masculinity can mean anything to anyone" (Gelfer, 2016, p. 279). While clearly implying masculinities performed by people on Earth, there is nothing in the formulation of Stage 4 that prevents its accommodation of the multiverse. The contradictory state of masculinities in superposition is also observable at Stage 4. For example, John Wayne might be a classic example of Stage 2 masculinity, assuming he believes this is how masculinity should look. But John Wayne can also exist quite happily at Stage 4 , assuming he believes this is how masculinity looks for John Wayne, but not necessarily anyone else. In the first situation of superposition-as-analogy, this works just fine, but if we take it any further, as soon as John Wayne is observed by a transfer of information his superposition collapses regardless of his intentionality (we note here that the intentionality of the subject in superposition does not seem relevant to either Tegmark or Butler).

Stage 5 is more interesting. At Stage 5, "masculinity exists as a consensual hallucination which nevertheless has many real effects" (Gelfer, 2016, p. 280). It is useful here to turn to Tegmark's discussion of reality. Tegmark describes external reality as "the physical world, which I believe would exist even if we humans didn't," consensus reality as "the shared description of the physical world that self-aware observers agree on," and internal reality as "the way you subjectively perceive the external reality" (p. 239). At Stages 1-3 on The Five Stages of Masculinity these three levels of reality are collapsed into one another.

At Stages 1-3, the masculinity the "I" perceives (internal reality) is perceived as the same as the masculinity that exists in external reality, and both Stage 2 and 3 (being both conscious of masculinity and having different worldviews) debate the consensus reality from their relative positions. Stage 4 is slightly different inasmuch as it has its internal reality model of masculinity, assumes the external model of reality is broad enough to 
accommodate all masculinities, and has little interest in debating the consensus reality (as consensus functions as a form of regulation that Stage 4 rejects). Stage 5 is the only stage where these three elements are starting to be teased apart (although not fully). It understands the differing natures of the three realities and begins to call them out: "masculinity exists as a consensual hallucination which nevertheless has many real effects" implies the interplay between these realities. But Stage 5 also questions the "I" in the first place, which aligns with another of Tegmark's conclusions, that "your perceptions of having a self, that subjective vantage point that you call 'I,' are qualia" (p. 318). Ultimately, Stage 5 masculinity retreats into certain mystical descriptions of the self not because it is necessarily "spiritual," but because mystical language sets a precedent for attempting to give form to that which lies beyond our current conceptual and linguistic frames of reference. It should then come as no surprise, given some of the parallels identified between Stage 5 masculinity, quantum masculinities and Tegmark's cosmology, that one of his critics observes that "the 'Mr. Hyde' part of the book crosses over to what I must consider science fiction and mysticism" (Frenkel, 2014). Ultimately, quantum masculinities and The Five Stages of Masculinity meet not in physics, but metaphysics.

More generally, from a structural point of view, another commonality can be identified between the multiverse and where The Five Stages of Masculinity "points." Stage 5 is not an endpoint of masculinities, indeed "Stage 5 is not a stage, rather a signpost to somewhere else" (Gelfer, 2016, p. 282). Visually, The Five Stages of Masculinity is rendered as a Venn pyramid, comprising five overlapping circles of decreasing size. The final and smallest circle representing Stage 5 is therefore not a final circle, rather the final circle that we can see. Remember, the other "parallel" universes in the Level I and II multiverse actually share the same space as us, they are just too far away for us to have seen their light yet (Level I) or are expanding away from us at such a speed that they will always be too far away for us to see their light (Level II). Alternatively, if you really must see what comes next, imagine the Stage 5 circle as a water-soluble pill with many small bubbles endlessly effervescing from the top: some are too small to see; others pop spectacularly; they are constantly in movement. 


\section{Conclusion}

The dual authorship of this article is not real. There is only one author; it is another thought experiment. The "single" author was born Joseph Organ but his name was changed at five years old to Joseph Gelfer. We like to imagine that Joseph Organ went on to follow his Stalinist namesake to take up a postdoc in theoretical physics at the Moscow Institute of Physics and Technology, while Joseph Gelfer went on to research masculinities and teach at a Catholic university in France. But of course, we do not need to like the idea of this happening, because somewhere in the multiverse it did happen: Joseph Organ never became Joseph Gelfer and he went to Moscow to study physics, as well as any number of other places and other subjects, and so did Joseph Gelfer. Indeed, Tegmark offers us - no doubt unwittingly — a modest precedent for these multiple personas with his confessed "Dr. Jekyll/Mr. Hyde Strategy": what is this if not a productive fracturing of the multiple self?

The shift to the "we" is a useful experiment. The life-art of Genesis Breyer P-Orridge provides a fine exploration of this concept. P-Orridge seeks to create an identity beyond the sex/gender binary through the psychic and physical transformation of pandrogyny. But P-Orridge also seeks to break a further binary: not just sex/gender, but the I/you: "we see the 'I' of our consciousness as a fictional assembly or collage that resides in the environment of the body" (P-Orridge, 2010, p. 445). This "fictional assembly" bears a commonality with the conclusions of Stage 5 and quantum masculinities, as well as Tegmark's endpoint of the "I" as qualia.

P-Orridge's shift results in a string of references to "we" and "our" that "they" maintain in all circumstances. At first glance, this pretense can feel annoying: it sounds like the Queen (and not a fun RuPaul kind of queen): "we are not amused." But when something feels troubling it is worth exploring why. The shift from the "I" to the "we" feels in some way subversive. It has the potential to resemble a political act that resists the atomization of society. It has the potential to recast "the massive subjective turn of modern culture" (Taylor 1991, p. 26), that infuses the individual and its subjective experiences with almost numinous gravitas, into a more dialogical process of meaning-making that we might call the "collective turn." It creates connections, it builds empathy, it mitigates isolation and 
loneliness, it obstructs the flow of the sentence: Who are "they" referring to? Who is speaking? We can now witness this multiplicity of identity occurring within the "individual" (to use the common frame of reference), society (inasmuch as what we experience on Earth through both history and the present) and the combined multiverse.

Intellectual honesty requires us to reveal our understanding of what is "real" about the proposed concept of "quantum masculinities" and what is not. We can say with certainty that quantum masculinities do not contain a shred of science. However, if we take Tegmark's multiverse seriously, quantum masculinities offer a scenario for describing the possibilities of multiple masculinities that is at least novel, and potentially "true" by the standards of external reality. Viewing multiple masculinities in a state of superposition is clearly farcical, yet we find ourselves having new thoughts about the differing nature of gender performance with or without an observer, and have another tool at hand to describe the often contradictory and paradoxical variables that arise when discussing masculinities.

Is there a difference between quantum masculinities and quantum femininities? Should this article actually be about "quantum gender"? Assuming the reality of the multiverse, we have to conclude that the idea of a gender binary is not just contestable but ludicrous in the context of an infinite number of outcomes. So in the same way that Stage 5 masculinity is identical to Stage 5 femininity (inasmuch as there being no such tangible and discreet thing as "masculinity" and "femininity"), so too quantum masculinities is the same as quantum femininities (inasmuch as "each individual [in the multiverse] dwells in a category of sex and gender as unique as their fingerprint").

However, throughout the multiverse there are an uncountable number of worlds just like ours where people who view masculinities via a Stage 5 lens nevertheless live in worlds where the majority of people are Stage 1 to 3, so they must always be tethered to these competing internal and consensus realties that are very much bound with "masculinity" and "femininity," in which case there does remain a difference between quantum masculinities and quantum femininities. Again, we find echoes of the state of superposition: it both is and is not the same.

In the introduction we noted that the study of masculinities is typically perceived as a modernist and sociological project, and agreed with Gottzén 
(2018) that this needed to be complemented with more postmodern and poststructural approaches. But we also posed the need to think bigger. In this article we have, sometimes tongue-in-cheek, put masculinities in dialogue with quantum theory and, in the process, have managed to surface a few new thoughts. All of gender studies — not just masculinities - is ripe for a massive leap forward in understanding itself. Sometimes this leap will occur due to connecting different existing fields of study and taking more seriously those liminal spaces where fields converge (such as the present conversation where physics bleeds into metaphysics), sometimes it will require waiting for certain scientific breakthroughs (we look forward to science finally cracking how consciousness works, for example, and engaging in a conversation about how this transforms the idea of the "self" in gender studies and beyond). The leap forward will also require being mindful about the way regulation takes places around knowledge production: the danger of constructing new orthodoxies at the same moment that we deconstruct the old, and of replacing hegemonies with a spectrum of micro-hegemonies. All of these challenges in the study of masculinities have-through the demands of infinite outcomes - already been fruitfully resolved in other parts of the multiverse: let's do the same here.

\section{References}

Boghossian, P., \& Lindsay, J. (2017). The conceptual penis as a social construct: A Sokal-style hoax on gender studies. Skeptic. Retrieved from https://www.skeptic.com/reading_room/conceptual-penis-socialcontruct-sokal-style-hoax-on-gender-studies

Barad, K. (1995). A feminist approach to teaching quantum physics. In S. V. Rosser (Ed.), Teaching the majority: Breaking the gender barrier in science, mathematics, and engineering (pp. 43-75). New York: Teachers College Press.

Beasley, C. (2013). Mind the gap? Masculinity studies and contemporary gender/sexuality thinking. Australian Feminist Studies 28(75), 108124.

Beasley, C. (2015) Caution! Hazards ahead: Considering the potential gap between feminist thinking and men/masculinities theory and practice. Journal of Sociology 51(3), 566-581. 
Bridges, T., \& Pascoe, C. J. (2014). Hybrid masculinities: New directions in the sociology of men and masculinities. Sociology Compass 8(3), 246-258. doi: https://doi.org/10.1111/soc4.12134

Brod, H. (1987). Introduction: Themes and theses of men's studies. In H. Brod (Ed.), The making of masculinities: The new men's studies (pp. 1-20). Boston: Allen \& Unwin.

Butler, J. (1999). Gender trouble: Feminism and the subversion of identity (2nd ed.). London: Routledge.

Christiaens, W. A. (2014). The Deleuzian concept of structure and quantum mechanics. In D. Aerts (Ed.), Probing the meaning of quantum mechanics: Physical, philosophical, and logical perspectives (pp. 189-208). Singapore: World Scientific Publishing.

Crockett, C. (2013). Deleuze beyond Badiou: Ontology, multiplicity, and event. New York: Columbia University Press.

Deleuze, G., \& Guattari, F. (1987). A thousand plateaus: Capitalism and schizophrenia (B. Massumi, Trans.). Minneapolis: University of Minnesota Press.

Frenkel, E. (2014). Ad infinitum: 'Our mathematical universe,' by Max Tegmark. The New York Times. Retrieved from:

https://www.nytimes.com/2014/02/16/books/review/ourmathematical-universe-by-max-tegmark.html

Gelfer, J. (2016). The five stages of masculinity: A new model for understanding masculinities. Masculinities and Social Change 5(3): 268-294. doi: http://dx.doi.org/10.17583/mcs.2016.2169

Gelfer, J. (2016b, March 7). Masculinity in 2016 appears to be in simultaneous states of extremity: increased normativity, increased queerness. [Update in Twitter]. Retrieved from https://twitter.com/drjosephgelfer

Gelfer, J. (2012). Will the real Joseph Gelfer please stand up: Multiple masculinities and the self. NORMA: International Journal for Masculinity Studies 7(2), 125-138.

Gelfer, J. (2009). Numen, old men: Contemporary masculine spiritualities and the problem of patriarchy. London: Routledge.

Gonsalves, A., Danielsson, A., \& Pettersson, H. (2016). Masculinities and experimental practices in physics: The view from three case studies. 
Physical Review Physics Education Research, 12, 020120. doi: https://doi.org/10.1103/PhysRevPhysEducRes.12.020120

Gottzén, L. (2018). Is masculinity studies really the 'odd man out'?

NORMA: International Journal for Masculinity Studies 13(2), 81-85.

doi: https://doi.org/10.1080/18902138.2018.1463089

Halberstam, J. (1998). Female masculinity. Durham, NC: Duke University

Press.

Harding, S. (1986): The science question in feminism. Milton Keynes: Open

University Press.

Hermans, H., \& Kempen, H. (1993). The dialogical self: Meaning as

movement. San Diego, CA: Academic Press.

Keller, E. F. (1985). Reflections on gender and science. New Haven, CT:

Yale University Press.

Krondorfer, B. (2017). God's hinder parts and masculinity's troubled

fragmentations: Trajectories of critical men's studies in religion.

Preprints and Working Papers of the Center for Religion and

Modernity 14. Münster: WWU Münster.

McConnell, A. R. (2011). The multiple self-aspects framework: Self-concept representation and its implications. Personality and Social Psychology

Review 15(1), 3-27. doi: 10.1177/1088868310371101

P-Orridge, G. (2010). Thee psychick bible: Thee apocryphal scriptures ov

Genesis Breyer P-Orridge and thee third mind ov thee Temple ov

Psychick Youth. Port Townsend, WA: Feral House.

Pettersson, H. (2011). Making masculinity in plasma physics: Machines,

labour and experiments. Science Studies 24(1), 47-65. Retrieved from https://sciencetechnologystudies.journal.fi/article/view/55269

Powis, D. (@dtpowis). (2016, March 7). Dibs on 'quantum masculinities.'

\#buzzwords \#jobmarket. [Update in Twitter]. Retrieved from

https://twitter.com/dtpowis

Schiebinger, L. (1999). Has feminism changed science? Cambridge, MA: Harvard University Press.

Sokal, A. (1996). Transgressing the boundaries: Towards a transformative hermeneutics of quantum gravity, Social Text 46/47, 217-252.

Retrieved from

http://www.physics.nyu.edu/sokal/transgress_v2/transgress_v2_single file.html 


$$
\text { MCS - Masculinities and Social Change, 7(3) } 229
$$

Taylor, C. (1991). The ethics of authenticity. Cambridge, MA: Harvard University Press.

Tegmark, M. (2014). Our mathematical universe: My quest for the ultimate nature of reality. New York: Alfred A. Knopf.

Woit, P. (2014). Book review: 'Our mathematical universe' by Max Tegmark. The Wall Street Journal. Retrieved from https://www.wsj.com/articles/book-review-8216our-mathematicaluniverse8217-by-max-tegmark-1389992755.

Joseph Gelfer is Lecturer at Université Catholique de l'Ouest, Angers, France

Joseph Organ is researcher at Moscow Institute of Physics and Technology Moscow, Russia

Contact Address: Direct Correspondence at Joseph Gelfer, Faculté des Humanités, Université Catholique de l'Ouest, 3 Place André Leroy, 49100 Angers, France, email: joseph @ gelfer.net 


\section{Hipatia Press \\ www.hipatiapress.com}

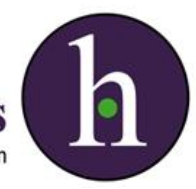

Instructions for authors, subscriptions and further details:

http://mcs.hipatiapress.com

\section{Comunicación de Marca en Instagram, ¿Una Cuestión de Género? El Rol del Influencer de Moda}

Raquel Martínez-Sanz \& Cristina Gonzalez Fernández ${ }^{1}$

1) Universidad de Valladolid, Spain

Date of publication: October $21^{\text {st }}, 2018$

Edition period: October 2018 - February 2019

To cite this article: Martínez-Sanz, R \& Gonzalez Fernández, C. (2018).

Comunicación de Marca en Instagram, ¿Una Cuestión de Género? El Rol del Influencer de Moda. Masculinities and Social Change,7(3), 230- 254. doi: 10.17583/MCS.2018.3693

To link this article: http://doi.org/10.17583/MCS.2018.3693

PLEASE SCROLL DOWN FOR ARTICLE

The terms and conditions of use are related to the Open Journal System and to Creative Commons Attribution License (CC-BY). 
MCS - Masculinities and Social Change Vol. 7 No. 3 October 2018 pp.230-254

\title{
Brand Communication on Instagram, a Gender Issue? The Role the Fashion Influencer
}

\author{
Raquel Martínez-Sanz \& Cristina González Fernández \\ Universidad de Valladolid
}

\begin{abstract}
Young people have found in the social networks the perfect space to inform and be informed. However, only a few have the ability to influence in purchasing decisions, lifestyle or preferences of the rest. The so-called social influencers have become an active part of brand communication, especially those dedicated to fashion. This research puts the focus on the strategies, resources and calls to participation used for the main Spanish fashion prescribers in their communication through Instagram. The gradual incorporation of men into this sector requires identifying the attitudes and forms of the connection -generating participation- or not taking into account the genre of the author and the type of values he projects. Through content analysis, the communicative activity of the 13 most outstanding characteristics is monitored in 2017. The results show a very similar use of recovery techniques, mainly promotional; dubious compliance with Spanish regulations on advertising - which obliges authors to explicitly state any message that promotes a product or service of a contractual relationship; and the use of the image to build idyllic lives from everyday scenes.
\end{abstract}

Keywords: influence marketing; influencer; social networks; brand communication; gender studies. 
MCS - Masculinities and Social Change Vol. 7 No. 3 October 2018

pp.230-254

\title{
Comunicación de Marca en \\ Instagram, ¿Una Cuestión de Género? El Rol del Influencer de Moda
}

\author{
Raquel Martínez-Sanz \& Cristina González Fernández \\ Universidad de Valladolid
}

\section{Resumen}

Los jóvenes han encontrado en las redes sociales el espacio perfecto para informar y ser informados. Sin embargo, solo unos pocos tienen la capacidad de influir en las decisiones de compra, estilo de vida o preferencias del resto. Los denominados influenciadores sociales han entrado a formar parte activa de la comunicación de marca, especialmente de las dedicadas a la moda. Esta investigación pone el foco de atención en las estrategias, recursos y llamadas a la participación utilizadas por los principales prescriptores de moda españoles en su comunicación a través de Instagram. La incorporación progresiva de hombres a este sector demanda identificar si las actitudes y formas de conectar - generar engagement- varían o no respecto al género del autor y el tipo de valores que proyecta. A través del análisis de contenido se realiza un seguimiento de la actividad comunicativa de los 13 perfiles más destacados en 2017. Los resultados evidencian una utilización muy pareja de técnicas de reclamo, fundamentalmente promocionales; un dudoso cumplimiento de la normativa española en materia de publicidad -que obliga a los autores a señalar de forma explícita todo mensaje que promocione algún producto o servicio procedente de una relación contractual-; y el empleo de la imagen para construir vidas idílicas a partir de escenas cotidianas.

Palabras clave: marketing de influencia; prescriptor; redes sociales; comunicación de marca; estudios de género. 



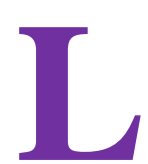

os entornos colaborativos 2.0 permiten al usuario narrar en primera persona tanto la experiencia de consumo como el trato recibido por las marcas. Al mismo tiempo, estas herramientas digitales nos acercan las valoraciones aportadas por otros consumidores con los que compartimos semejantes intereses. El boca-oreja lejos de desaparecer se ha visto incrementado con las redes sociales al ampliar el círculo de confianza de las personas que ya no tienen que estar necesariamente próximas físicamente (Fresno, Daly \& Segado, 2016).

Si hay un colectivo especialmente vinculado con esta forma de proceder es el público joven, los denominados Millennials (Ruiz Cartagena, 2017) quienes encuentran en el espacio digital la plataforma perfecta para dar a conocer su forma de vida, sus conocimientos u opiniones al respecto de temas sobre los que se sienten legitimados.

Sin embargo, solo un pequeño porcentaje de ellos demuestran tener la capacidad de influir, es decir, de generar una reacción en sus seguidores y, en consecuencia, que sus recomendaciones, vivencias y opiniones se posicionen como una opción de referencia frente al resto (González-Romo, García-Medina, \& Macdonald, 2016). Esta cualidad unida al interés que su comunicación suscita, y que se traduce en una voluminosa comunidad de usuarios, da lugar a lo que se conoce como influenciador social-influencer.

Ese liderazgo patente en determinados jóvenes genera que muchas marcas comerciales, y muy especialmente las ligadas al ámbito de la moda y la belleza, encuentren en los perfiles de estos líderes el escaparate perfecto para entrar, de forma no intrusiva, en el imaginario de su público objetivo y contagiarse de la credibilidad y confianza que irradia el influencer. A través del marketing de influencia se establecen las condiciones que van a regular la relación profesional entre marca y prescriptor (Uzunoğlu \& Kip, 2014) haciendo alusión directa, entre otras cuestiones, a las acciones, los tiempos o la remuneración. Sin embargo, la legislación española es clara al respecto, el receptor tiene que poder distinguir los mensajes pagados, y en consecuencia de carácter publicitario, de los que no lo son, aunque estén firmados por particulares (Martínez \& Gaona, 2016).

Esta investigación focaliza su interés en la industria de la moda, una de las más rentables a nivel mundial y que mayor poder de atracción suscita entre los jóvenes (Ribeiro-Cardoso, Teixeira \& Santos, 2016; Pérez, Clavijo, Luque \& Pedroni, 2017). Bajo este marco, se establece como principal 
objetivo examinar de qué forma los principales influencers de moda en España llevan a cabo una comunicación de marca en sus perfiles de Instagram, una red social que en los últimos años ha visto aumentar tanto sus usuarios de entre 16-30 años como la estima asignada, especialmente entre el colectivo de mujeres (IAB Spain, 2015 y 2016).

Si la fórmula del influenciador social se resume en disponer de una numerosa comunidad de seguidores y de capacidad para interferir en las decisiones de terceros relativas principalmente a gustos, interés y/o deseos (Booth \& Matic, 2011) este trabajo se interesa por identificar las estrategias desarrolladas por estos líderes de opinión (igers ${ }^{1}$ ) orientadas a aumentar su comunidad y su capacidad de influencia. Asimismo, y de manera transversal, se pretende observar si la comunicación (temas, lenguaje, tono, imágenes, etc.) difiere entre hombres y mujeres influencers o si por el contrario existe una conducta homogénea válida para ambos sexos.

En consecuencia, la resolución de estos objetivos invita a responder a las siguientes preguntas de investigación:

- ¿En qué medida se identifica a los mensajes/acciones pactadas con la marca (marketing de influencia) de los que son creados por iniciativa y convencimiento propio por los prescriptores de moda? El propósito es averiguar si los prescriptores informan a su comunidad del establecimiento de un vínculo profesional -puntual o continuo- con una marca y cómo se evidencia.

- ¿Qué peso tiene la imagen y el texto en la comunicación ejercida por el influenciador a través de Instagram? A través de este interrogante se valora el papel que juega cada uno de estos componentes en esta red social eminentemente visual.

- ¿Existen diferencias entre las técnicas empleadas por mujeres prescriptoras de moda y sus homólogos hombres al utilizar su canal de Instagram? Se trata de conocer si la comunicación y forma de proceder de los influencers varía en función del género.

Numerosa literatura demuestra que en pleno siglo XXI los roles de género, además de estar fuertemente interiorizados, se perpetúan a través de los medios masivos (Lomas, 2005; Chicharro Merayo, 2013; Ferreiro Habra, 2018) transmitiendo inquisitivamente lo que se espera del hombre y de la 
mujer. Descubrir el papel que juega esta red social, utilizada prioritariamente por jóvenes, para dar continuidad a los valores, prejuicios e ideales estandarizados para cada género, se convierte en la pregunta transversal a todo el estudio que de demostrarse corroboraría el poder de la imagen (Sartori, 1998) y la necesidad de una pedagogía de la mirada (Belmonte y Guillamón, 2008).

\section{La Evolución del Fenómeno Influencer}

La figura del influenciador social nace con la eclosión de la Web 2.0. Los foros fueron el primer escenario digital abierto al intercambio de pareceres aunque pronto la conversación se trasladó al blog, un espacio mejor estructurado que cultivó todo tipo de temáticas e intereses y que incorporó otras posibilidades expresivas además del texto (Martínez-Sanz, 2013). La variante del ego-blogger - bitácora dedicada en exclusiva a dar a conocer las vivencias personales protagonizadas por su autor- resultó la avanzadilla de algunas de las características propias de los influencers de moda más jóvenes (Uzunoḡlu \& Kip, 2014).

Tal y como explica Gillin (2009), la aparición de Google AdSense en 2003 marcó un punto de inflexión al introducir la variable económica y por tanto lucrativa en la actividad de los blogs. La publicidad puso bajo su punto de mira a los blogs más visitados y mejor posicionados iniciándose así la carrera de las marcas por aparecer en los espacios web más demandados en función de la concordancia temática. La segmentación de los públicos se convirtió en una necesidad y en un claro factor de éxito en cualquier campaña publicitaria (Rojas \& Redondo, 2017). Los blogs también sirvieron para relanzar el marketing de contenidos al descubrir que las entradas actualizadas, fundamentadas en el dominio de la materia y en la preocupación por cubrir necesidades prácticas de los públicos, convertían a su autor en fuente de referencia.

Sin embargo, han sido las redes sociales las que han llevado el fenómeno influencer a su punto más álgido, sirviendo de trampolín profesional para muchos jóvenes cuyos estilismos, sugerencias y compras sirven de inspiración al resto (Ribeiro-Cardoso et al., 2016; González-Romo et al., 2016). Desde un punto de vista comercial se tiene pleno convencimiento de que la recomendación procedente de una fuente en la que se confía resulta un 
factor decisivo a la hora de adquirir un producto o servicio (Bigné, Küster \& Hernández, 2013; Benítez-Eyzaguirre, 2016). A todo esto hay que añadir que las redes sociales no se entienden sin interacción, sin la participación activa del receptor. Esto provoca que el consumidor pueda asumir al mismo tiempo el rol de creador, contribuyendo con sus reacciones a moldear la evolución de la marca (Castelló, 2010).

Uno de los primeros y más destacados casos de marketing de influencia en el ámbito de la moda fue puesto en marcha en 2015, por la firma estadounidense Lord and Taylor (Imagen 1). Esta marca solicitó a 50 destacadas instagramers subir una foto el mismo día y a la misma hora con un vestido concreto de su nueva colección, logrando que dicha prenda se agotara a los pocos días. Con esta iniciativa, la marca logró que el vestido fuese visto por las consumidoras como un must-have generándose en ellas la necesidad de adquirirlo. Con este ejemplo la industria comprendió que la capacidad de convicción es poderosamente superior si procede de una voz cercana, en la que se confía previamente que la que puede tener una marca comercial a pesar de sus esfuerzos publicitarios.
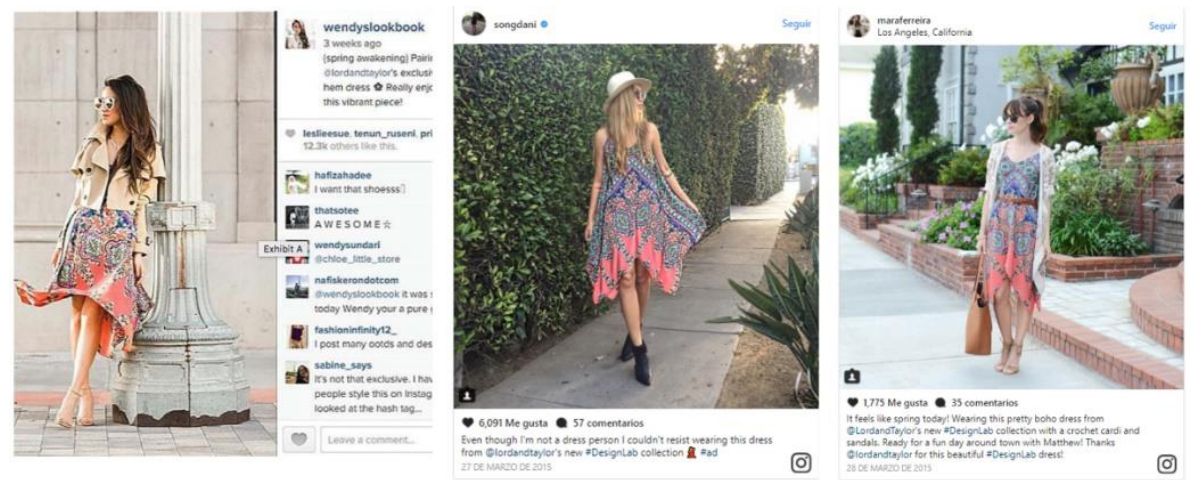

Imagen 1. Publicación de varias instagramers con el vestido distribuido por Lord and Taylor. En perfil de @wendyslookbook; @songdani; @ maraferreira

\section{El Poder de Atracción del Prescriptor}

Si hay algo que caracteriza a todos los influencers es su activa presencia en redes sociales, lo que les lleva a no limitar su actividad a una sola sino a 
expandir y adaptar su mensaje a las diferentes plataformas. El dominio y especialización de cada una de ellas ha derivado en denominaciones como: instagramer, youtuber, tuitero, etc. para referirse al usuario generador de contenido de cada espacio.

Asimismo, el influenciador social es una persona con una amplia comunidad de seguidores que estiman la información suministrada por este con quien comparten intereses comunes. Por lo que respecta a su comunicación, las posibilidades resultan muy variadas: desde mensajes puramente informativos, entre los que se incluyen las recomendaciones o consejos prácticos, al relato de experiencias, formas de vida u opiniones. Incluso lo relativo al lenguaje abarca un gran abanico de posibilidades: texto, imagen fija, emojis, vídeos, etc. En definitiva, lo que se trata es de conectar y de aportar al público lo que busca.

La investigación llevada a cabo por Wiedmann, Hennings y Langner (2010) señala las cualidades más determinantes de los perfiles que aspiran a lograr influencia social en el ámbito de la moda.

- Sintonía con los usuarios en términos de edad, género y ubicación. Los internautas tienden a interactuar y a entablar relaciones sociales con las personas con las que comparten características comunes.

- Vinculación con la moda.

- Experiencia y conocimientos específicos.

- Carácter innovador. Interés por ser de los primeros en probar, acudir o experimentar para después narrarlo en primera persona.

- Personalidad arriesgada. Se trata de personas sin miedo a los cambios que no ponen ningún tipo de limitación a la hora de aplicarlos sobre ellos mismos.

Un importante porcentaje de los seguidores de estos líderes de opinión son jóvenes (IAB, 2016), integrantes de la denominada Generación Millennial (Ruiz Cartagena, 2017). Este colectivo, nacido entre los años 1981 y 2000, no entiende el mundo sin Internet; es más, la Web representa una de sus principales fuentes de información, además, de ser los principales impulsores del consumo en línea. Al hilo de esto McCormick (2016) explica una forma muy peculiar de proceder de los milenials: identifican cada producto a partir de una marca (hamburguesa - McDonalds; cereales - Kellogs; coche de lujo 
- Ferrari, etc.) estableciendo con ellas una relación de autorepresentación y favoreciendo que las marcas se conviertan en una extensión de ellos mismos. Sin embargo, y tal y como han descubierto Pedro Rojas y María Redondo (2017) los jóvenes no hablan con las marcas; apenas se interesan por interactuar con ellas. De ahí la necesidad de encontrar otros mecanismos para que las empresas impacten sobre sus públicos objetivos. Para suplir esta carencia el marketing de influencia ha demostrado ser una herramienta eficaz y rentable para generar engagement (Aswani, Ghrera, Kar \& Chandra, 2017; Castelló \& Pino, 2017). Las cifras lo avalan y un 65\% de los profesionales de la moda aseguran haber llevado a cabo acciones o campañas con influencers en 2016 (Launchmetrics, 2017). No es casualidad que el fenómeno influencer haya nacido en plena crisis económica al demostrar que obtiene mejores resultados que la publicidad tradicional y a un menor coste, sin olvidar la posibilidad de las marcas de controlar el retorno de su inversión y llevar un seguimiento de sus ventas derivadas.

\section{Planificar y Orientar la Influencia en el Ámbito Digital. La Imagen como Transmisora de Identidades de Género Dominantes}

El marketing de influencia consiste en el contacto y vínculo que establece una empresa con usuarios influyentes de la red -blogguers, instagramers, youtubers, celebrities, etc.- previamente identificados, para multiplicar el alcance de su comunicación de marca. El marketing de influencia fusiona la comunicación corporativa con la publicidad extrayendo lo mejor de cada una para, en última instancia, generar un vínculo con el usuario que acabe fidelizándolo (Castelló \& Pino, 2015).

Además, esta relación supone un beneficio mutuo para todas las partes: mientras que la marca se impregna de la credibilidad y confianza que irradia el prescriptor, este ve recompensado su trabajo fortaleciendo su posición y relevancia profesional ya que todo el contenido, de carácter orgánico -y por tanto, no pagado-, unido a la interacción de los usuarios, contribuye a mejorar el posicionamiento web y con ello su visibilidad. Para alcanzar tan óptimos resultados es fundamental que empresa e influencer compartan valores y formas de hacer y así dotar de naturalidad a la comunicación. Se trata de que el esfuerzo quede canalizado hacia una misma dirección y no se produzcan contradicciones. 
En la mayoría de las ocasiones el primer encuentro con la marca se produce cuando la empresa, convencida de la calidad de sus productos o servicios, se muestra abierta a que personas imparciales, especializadas en el sector lo prueben, manipulen y/o experimenten. No es extraño, por tanto, que muchos influencers reciban muestras gratuitas o sean invitados a determinadas presentaciones o desfiles con el fin de que estos hagan una mención posterior en sus redes, trasladando a su comunidad de seguidores la experiencia, el trato y/o las impresiones vividas (Venegas, 2015). La finalidad no es solo conseguir un comentario que recomiende la adquisición de una determinada prenda o complemento sino convencer al líder de opinión de las bondades de la marca y que, de forma natural, se convierta en embajador de ella.

La relación profesional con la empresa sube de nivel y linda con la publicidad cuando el influenciador pacta, a cambio de una retribución, el tipo de mensaje a publicar, el sesgo que le tiene que aplicar a su comentario claramente positivo-, y la regularidad; todo con el fin de afianzar los atributos del producto. Este nuevo estatus suprime la libertad del influencer para hacer un juicio objetivo de su parecer. A este respecto, la legislación española es clara: el receptor debe contar con evidentes signos que separen los mensajes informativos de los publicitarios; de lo contrario se incurre en publicidad engañosa (Ley General de Publicidad, 1988; Ley de Competencia Desleal, 1991).

Tal y como demuestran numerosas investigaciones, el uso de la imagen en los medios de comunicación masivos -publicidad, cine o televisión- ha resultado clave para expandir y normalizar lo que se espera que han de ser, comportarse y pensar los hombres y las mujeres para ser socialmente aceptados (Belmonte Arocha, 2014; Ferreiro Habra, 2018). En definitiva, la cultura de masas, subordinada por la visión patriarcal, ha construido una identidad de género dominante para lo masculino y lo femenino que ridiculiza y rechaza lo diferente y que, al ejercerse, produce una violencia simbólica (Belmonte \& Guillamón, 2008).

En este escenario se observa como la publicidad, lejos de volcarse en ensalzar los atributos tangibles del producto promocionado, trabaja en la conexión imaginaria de los valores que alcanzará el potencial comprador en el momento en el que se adquiera. En consecuencia, proliferan los anuncios que ensalzan determinados estilos de vida, corporalidades o pensamientos alineados con lo dominante: "se vende un oasis de ensueño, de euforia y de 
percepción absoluta" (Lomas, 2005, p.269) que sin embargo, y como reconoce el mismo autor, genera frustraciones, inseguridades y dolor, ya que solo un pequeño porcentaje se adecua al modelo hegemónico no sin sacrificios y renuncias (Lomas \& Arconada, 2003).

La noción de masculinidad se ha definido tradicionalmente por oposición, por lo que son frecuentes las muestras que tratan de evidenciar su distanciamiento de lo femenino y lo homosexual, principalmente a través de la exhibición de la fuerza, el desafío a la autoridad o el rechazo hacia lo emocional (Belmonte \& Guillamor, 2008). El trabajo de Ferreiro Habra (2018) determina que el modelo publicitario ideal es el del hombre joven, blanco, heterosexual, urbano, de clase media o alta, profesional y deportista mientras que, y a través de la ficción televisiva, Chicharro Merayo (2013) identifica la feminidad con un tipo de mujer vulnerable que necesita sentirse reafirmada; que hace un uso instrumental del sexo, el erotismo y la estética; y que reivindica su espacio en el ámbito laboral.

\section{Metodología}

Si como se ha explicado, el influencer trata de fidelizar y expandir su comunidad de usuarios a través de la comunicación en redes sociales, este trabajo persigue analizar las técnicas específicas empleadas por distintos líderes de opinión en moda a través de sus respectivas cuentas en Instagram. Para ello, se realizó una investigación de tipo analítico-descriptiva en la que se estudian las publicaciones e interacciones logradas por 13 destacados influencers de moda españoles desde un punto de vista cuantitativo y cualitativo.

El análisis de contenido resulta la técnica más adecuada para el objetivo planteado por su capacidad para combinar la observación y producción de datos, con su interpretación (Krippendorff, 2004). Para asegurar la rigurosidad del método se elaboró una plantilla de análisis que pone el acento tanto en los aspectos visuales y textuales del mensaje como en las interacciones recibidas. La plantilla estuvo compuesta por cuatro apartados:

- Composición. Se específica a partir de qué elemento gira la publicación: fotografía inédita, imagen de archivo, gif o vídeo; en qué momento del día se subió a Instagram: por la mañana, por la tarde o por la noche; y el día de la semana. 
- Repercusión. Se cuantifica el número de me gusta y comentarios recibidos en las primeras 24 horas.

- Contenido visual. Se identifica al influencer en términos de presencia o ausencia y la condición del protagonista: persona, objeto o paisaje. Asimismo, nos interesamos por el tipo de plano utilizado: general, medio, selfie, americano o plano detalle por las implicaciones que conlleva; cómo se ha producido la captura: de forma espontánea o preparada; qué refleja la escena: momento personal, estado de ánimo o vida profesional; estética; si aparecen de forma explícita marcas comerciales; si se integran etiquetas dentro de la imagen y de qué tipo; y alusión a la localización.

- Contenido textual. Se señala el idioma(s) empleado(s), si aparecen emojis en la configuración del mensaje textual y si hay mención a otros perfiles: empresas o personas. Además, se cuantifica y clasifica la semántica de los hashtags y si se expresan de forma clara las colaboraciones comerciales entre marca e influencer.

Por último, y valorando al mensaje en su conjunto (imagen más texto) se especifica la intencionalidad.

Para llevar a cabo la selección de los influencers, se tomó en cuenta tres aspectos: 1. La pertenencia a una agencia de influenciadores en España 2. La vinculación directa y tangible con el ámbito de la moda 3 . El volumen de su comunidad digital. La muestra seleccionada incluyó los dos géneros: mujeres (54\%) y hombres (46\%) con un porcentaje no paritario ya que la presencia de mujeres en el ámbito de la prescripción de moda es considerablemente mayor. A pesar de todo, se observa que la incorporación del hombre resulta progresiva. Esta situación motiva que nuestro estudio, entre otros asuntos, se interese por reconocer si existen estrategias de influencia diferenciada entre hombres y mujeres para conectar con la comunidad de seguidores.

Tal y como recoge la Tabla 1, la muestra definitiva quedó constituida de la siguiente manera: 


\section{MCS - Masculinities and Social Change, 7(3) 241}

\section{Tabla 1}

Rasgos principales de los influencers de moda analizados

\begin{tabular}{|c|c|c|c|c|}
\hline Género/ Nombre & Perfil & Seguidores $^{2}$ & Agencia & $\begin{array}{c}\text { Publicaciones } \\
\text { emitidas/anali } \\
\text { zadas }\end{array}$ \\
\hline F. María Turiel & @meryturiel & 391.000 & Go Talents & $47 / 14$ \\
\hline F. Inés Arroyo & @ines_arroyo & 342.000 & Okiko Talents & $22 / 9$ \\
\hline F. María Valdés & $@$ marvaldel & 263.000 & Go Talents & $33 / 7$ \\
\hline M. Luis J. García & @lewisonn & 224.000 & $\begin{array}{l}\text { Influence } \\
\text { Code }\end{array}$ & $5 / 3$ \\
\hline F. Marta Soriano & @msorianob & 145.000 & Go Talents & $34 / 10$ \\
\hline M. Alejandro More & @ alejandrojamo & 135.000 & $\begin{array}{l}\text { Influence } \\
\text { Code }\end{array}$ & $12 / 1$ \\
\hline F. Adriana Boho & @adrianaboho & 115.000 & Influgency & $31 / 7$ \\
\hline F. Marta Vidaurreta & $\begin{array}{l}\text { @ martavidaurre } \\
\text { ta }\end{array}$ & 108.000 & Go Talents & $19 / 4$ \\
\hline $\begin{array}{ll}\text { M. } & \text { Aarón } \\
\text { Fernández } & \end{array}$ & $\begin{array}{l}\text { @ aaronfernande } \\
\text { zmoda }\end{array}$ & 74.100 & Hamelin & $16 / 5$ \\
\hline F. Sara Domenech & @ saraemdi & 73.400 & Influgency & $31 / 6$ \\
\hline M. Miguel Carrizo & @ miguelcarrizo & 39.500 & Okiko Talents & $37 / 7$ \\
\hline M. Gaby Rope & @ gabyrope & 31.700 & Influgency & $5 / 1$ \\
\hline $\begin{array}{ll}\text { M. } & \text { Fernando } \\
\text { Montoya } & \\
\end{array}$ & $\begin{array}{l}\text { @ montoya_nan } \\
\text { do }\end{array}$ & 28.500 & Influgency & $18 / 3$ \\
\hline
\end{tabular}

El estudio de los perfiles se produjo en el mes de abril de 2017, analizándose con detalle todos los mensajes publicados a lo largo de siete días no correlativos y aleatorios. Para garantizar, además, la aleatoriedad y evitar coincidencias en el día de la semana, se adoptó como criterio la selección de los días múltiplos de cuatro. En consecuencia, las fechas en las que se recogió el corpus de análisis fueron las siguientes: 4 de abril (martes), 8 de abril (sábado), 12 de abril (miércoles), 16 de abril (domingo), 20 de abril (jueves), 24 de abril (lunes) y 28 de abril (viernes).

De un total de 311 publicaciones emitidas por los 13 especialistas en abril de 2017, el corpus de estudio se compuso de 77 mensajes cuyo impacto número de comentarios y me gusta recibidos- se registró a las 24 horas de haber sido publicados a través de capturas de pantalla. 


\section{Resultados}

Desde un punto de vista cuantitativo se observa que los influenciadores con más seguidores fueron los que más mensajes publicaron, aunque es preciso matizar que el volumen de actividad fue muy superior -casi el doble- en los perfiles de mujeres que de hombres. Para ellas, la media relativa a todo el mes se situó en 31 entradas -una por día- mientras que ellos publicaron 16 mensajes al mes, lo que representa, aproximadamente, una publicación cada dos días.

Los mensajes, en el $98,7 \%$ de los casos, estuvieron configurados por imágenes inéditas lo que supone priorizar la fotografía y el estilo personal de cada autor pero, al mismo tiempo, relegar la experimentación con el resto de formatos disponibles tales como vídeos, gifs o memes, de gran aceptación social. Los expertos en moda eligen los domingos y la franja horaria de la noche como el momento preferido para subir contenido.

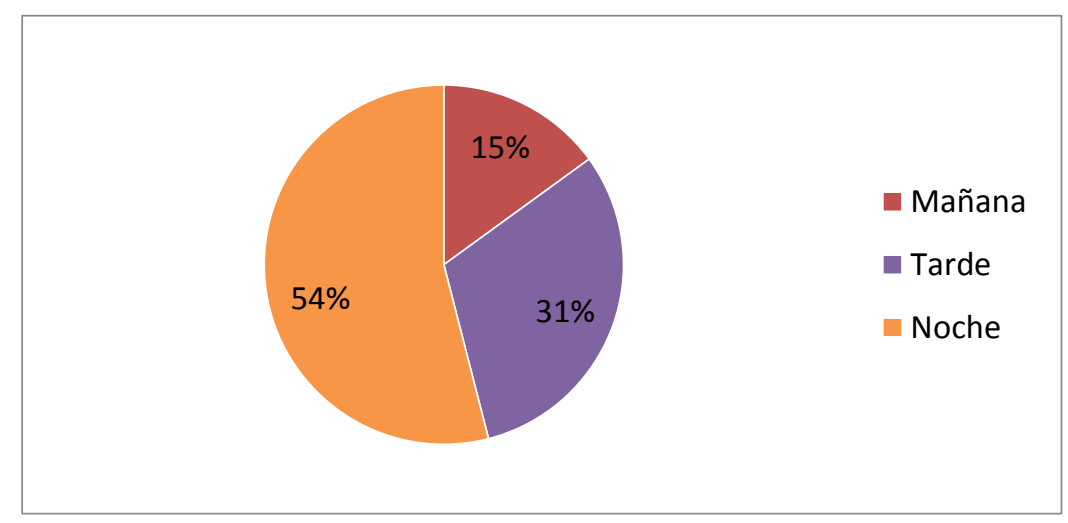

Figura 1. Momento del día en el que se emiten las publicaciones

Fuente. Elaboración propia

En cuanto al alcance logrado por cada publicación, si bien es cierto que no es posible indicar el número de visualizaciones obtenidas, sí se ha podido registrar el número de likes que cada mensaje alcanzó en las primeras 24 horas, constatándose con ello que no solo fue visto sino que resultó lo suficientemente del agrado del seguidor como para merecer el gesto de 
pinchar sobre me gusta. Las 77 publicaciones recibieron un total de 580.503 muestras de conformidad, siendo @meryturiel la más aclamada con diferencia.

\section{Aspectos Visuales}

Por lo que respecta a las fotografías -núcleo central del mensaje en Instagram, el influencer aparece en nueve de cada diez imágenes. Dato que desciende ligeramente si solo nos fijamos en los perfiles masculinos (85\%). Los prescriptores estudiados aparecen como protagonistas absolutos de la imagen en el $88 \%$ de los casos. En el resto de situaciones, o comparten el protagonismo o éste recae íntegramente en un paisaje o en un objeto. Para estos últimos, lo que más se repite son los complementos de moda: reloj de pulsera o gafas de sol. Los atributos que predominan en las imágenes en las que el influencer estuvo ausente y el protagonismo recae en algún lugar o paisaje son el relax y la calma, mientras que cuando la escena se centra en determinado objeto se ensalzan cualidades como la elegancia y la comodidad.

El tipo de plano más utilizado es el general, identificado en la mitad de las imágenes y dirigido a mostrar al influenciador de cuerpo entero, lo que le permite exhibir sus looks completos. En un $21 \%$, el plano es de tipo medio, uno de los más utilizados en la fotografía de moda porque descubre hasta la cintura, permitiendo ver el rostro de cerca y la prenda superior que se porta. En la misma proporción (21\%) se opta por el plano americano que deja percibir hasta las rodillas, por lo que resulta una buena opción cuando se busca dar importancia a complementos como bolsos o mochilas. Las selfies (autorretratos) solo se presentan en un 3\% de las imágenes. Este formato, en el que solo tiene cabida la representación de la cara, apenas permite la exhibición de prendas o complementos por lo que su uso resulta muy residual. El 4\% restante se corresponde con el plano detalle utilizado exclusivamente para mostrar objetos concretos como relojes o pulseras portados por ellos mismos.

Un rasgo presente en todas las imágenes, independientemente del género de su creador, es su evidente preparación previa. Con el objetivo de no desviar la atención del internauta, buena parte de las fotografías se realizan sobre fondos básicos -generalmente paredes o puertas- concentrando el interés por el outfit del influenciador. Su pose trata de transmitir espontaneidad y 
naturalidad, aunque nada de lo que aparece es casual. A pesar de todo, y con una clara intención de conectar con los seguidores, se escenifican situaciones cotidianas y sencillas, vinculadas con el día a día de los jóvenes como excusa para mostrar qué ropa llevan.

En los perfiles de mujeres abundan las fotografías que capturan momentos personales como es el hecho de maquillarse, pasear o disfrutar de una copa. El movimiento simulado de brazos o piernas y sobre todo orientar la mirada hacia el horizonte transmite la sensación de haber sido capturadas en plena acción. En el caso de los hombres, el posado es más evidente dirigiendo su mirada hacia el objetivo de la cámara.

El hecho de que todos los instagramers analizados formen parte de una agencia de publicidad especializada permite deducir que están interesados en la promoción de marcas. Si bien es cierto que no podemos asegurar que en el momento de realizar el análisis el influenciador recibiera una contraprestación económica por ello, sí se advierte que la mayoría de las publicaciones (82\%), además de exhibir prendas, complementos de moda o espacios físicos, nombran a las marcas a través de etiquetas en el interior de la imagen, para reforzar el mensaje y eliminar cualquier tipo de duda sobre la procedencia (Imagen 2). Hay que reconocer que de no hacerlo, solo en un $18 \%$ de los casos sería posible reconocer qué o quién comercializa el producto exhibido a través de la identificación del isotipo, isologo o imagotipo representativo. Valga de ejemplo, la aparición de un polo con un cocodrilo para asociar de forma automática que esa prenda pertenece a la firma Lacoste. Sin embargo, esto no es lo habitual por lo que se requieren más evidencias para dar a conocer el lugar o producto. Esta circunstancia se reafirma al observarse que la mayor parte de los influenciadores, tanto hombres como mujeres realizan una doble promoción: visual y textual. 

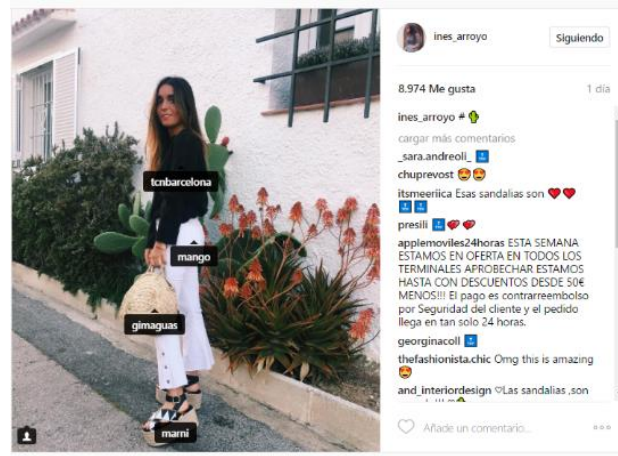

Imagen 2. Fotografía con etiquetas que identifica marcas

A pesar de todo, si hay una pauta común en ambos géneros es el cuidado por la puesta en escena de sus imágenes, incluso cuando ellos no aparecen, lo que se manifiesta especialmente en la colocación y orden de los elementos y en la armonía cromática, pudiendo llegar a ver imágenes que destacan por su calidad artística.

\section{Aspectos Textuales}

Aunque Instagram es una red social eminentemente visual admite espacios para la escritura como son el título de la publicación, las etiquetas de la fotografía y los comentarios.

Todas las publicaciones salvo una llevaban un título propio: cerca de la mitad $(49 \%)$ redactado íntegramente en castellano mientras que un 5\% además, incorporó alguna expresión anglosajona a lo largo del mensaje. Un $27 \%$ fue escrito en su totalidad en inglés y un $12 \%$ en ambos idiomas, circunstancia, esta última, tan solo presente en perfiles gestionados por hombres (Tabla 2). En todo caso, la aparición de un segundo idioma confiere al perfil una imagen más profesional y accesible. En el $6 \%$ restante, y presente tan solo en el muro de las mujeres, el título se compuso únicamente por emojis. A pesar de esto, se observa que los emojis son un recurso habitual empleado por los influencers de ambos géneros, pues en el $76 \%$ de los mensajes se aprecia la presencia de al menos uno. 


\section{Tabla 2}

Composición del título según el género del perfil

\begin{tabular}{lcc}
\multicolumn{1}{c}{ Título } & $\begin{array}{c}\text { Perfiles } \\
\text { femeninos }\end{array}$ & $\begin{array}{c}\text { Perfiles } \\
\text { masculinos }\end{array}$ \\
\hline Íntegramente en castellano & $48 \%$ & $50 \%$ \\
Con algunas expresiones en inglés & $5 \%$ & $5 \%$ \\
Íntegramente en inglés & $34 \%$ & $20 \%$ \\
En castellano e inglés & $0 \%$ & $25 \%$ \\
Compuesto únicamente por emoji(s) & $12,5 \%$ & $0 \%$ \\
Compuesto por texto y emoji(s) & $79 \%$ & $70 \%$ \\
Incluye hashtags & $46 \%$ & $90 \%$ \\
\hline
\end{tabular}

También con una gran aceptación se encuentra el empleo de hashtags. Etiquetas que contribuyen a mejorar la descripción de la imagen y a organizar las publicaciones según su contenido. Gracias a su capacidad para potenciar la visibilidad de la publicación, muchas marcas crean hashtags propios aplicados a sus productos o establecimientos. Aclarado esto, esta investigación detecta que dos de cada tres publicaciones redactadas por influencers de moda incorporan este recurso que se destina a mencionar principalmente, y en este orden: al producto exhibido (\#clusewatches, \#aniversariodesalia); a la marca mediante la alusión a su nombre (\#mango, \#garnier, \#RimmelGirl); al estado de ánimo del influenciador (\#myspringmoments, \#vivolibremente); a un lugar concreto (\#Andorra, \#Asturias) y a un sitio web (\#blog \#YouTube).

Otro recurso valioso que Instagram admite en los títulos de las publicaciones es mencionar a otro perfil -valga como ejemplo: "muchas gracias a@Converse por estas zapatillas"- que posibilita al usuario ser redireccionado a un nuevo espacio. Se observa que los perfiles masculinos son más proclives a utilizarlo, pues está presente en nueve de cada diez publicaciones, mientras que en las mujeres solo encontramos la alusión a otra cuenta en la mitad de los mensajes. Esta prestación es utilizada por los influencers de moda para redirigir a marcas comerciales y en menor medida a perfiles personales.

Un uso también desigual presenta la mención a la localización desde donde se ha tomado la fotografía. Esta información se sitúa en la cabecera, junto al 
nombre del autor de la publicación y es visible en el caso de los chicos en el $80 \%$ de sus entradas, mientras que en el de las chicas no supera el $18 \%$. Para estas últimas, se evidencia que el espacio físico es un elemento accesorio aunque cuando se insinúa siempre es para ensalzarlo de forma explícita. Sesgo positivo que también trasladan los perfiles masculinos. Circunstancia que resulta un indicio más para suponer que se ha establecido una relación profesional. Sirva como ejemplo, la sesión de fotos que realiza @ montoya_nando en la tienda de ropa Forecast (Imagen 3).

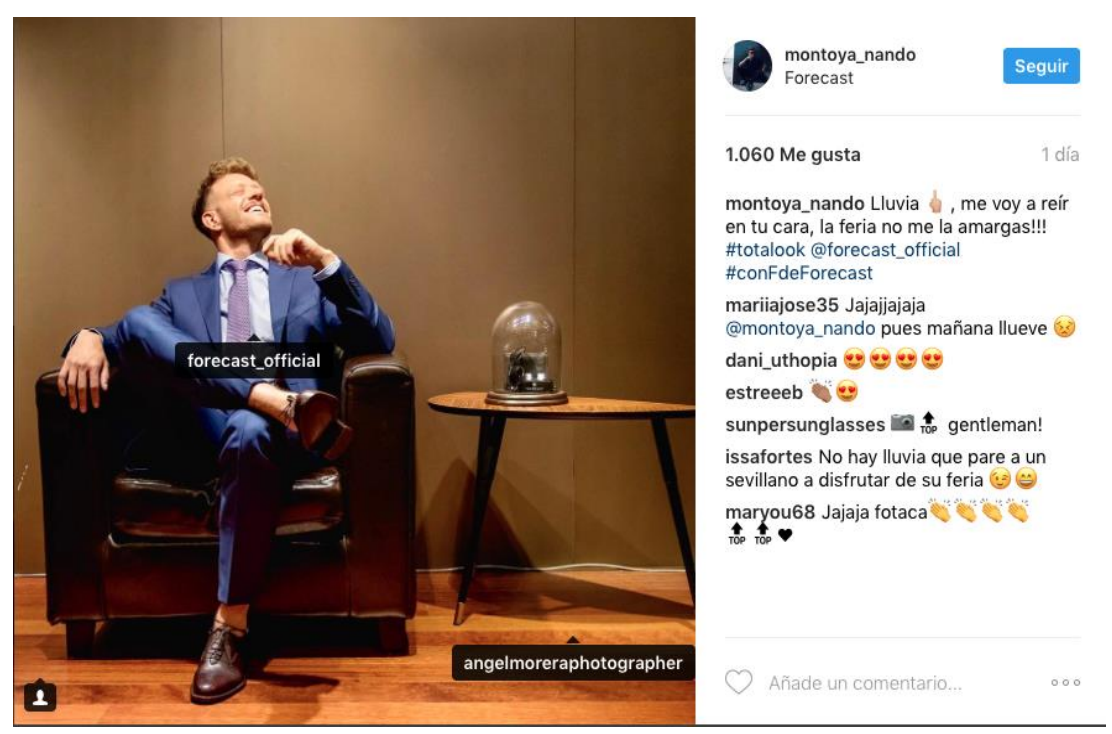

Imagen 3. Publicación que refuerza la localización

Por lo que respecta a la intencionalidad, y tomando como referencia al mensaje en su conjunto, se observa que el objetivo del $80 \%$ de las publicaciones de los influencers masculinos y del $49 \%$ de las publicaciones femeninas es el de promocionar de forma explícita una prenda, un espacio de moda o un producto. El modo de proceder en esta situación es bastante semejante en ambos géneros: el artículo o espacio físico aparece en la imagen acaparando la máxima atención mientras que el texto no escatima en halagos incluyendo, además, la mención directa a la marca mediante hashtags y/o a su 
cuenta en Instagram (Imagen 4). En estos casos, sin duda, los más próximos a una supuesta vinculación profesional entre influencer y marca, no se advirtieron señales explícitas que confirmaran una posible colaboración remunerada entre las dos partes. Hecho que de acaecer estaría contraviniendo la ley española.
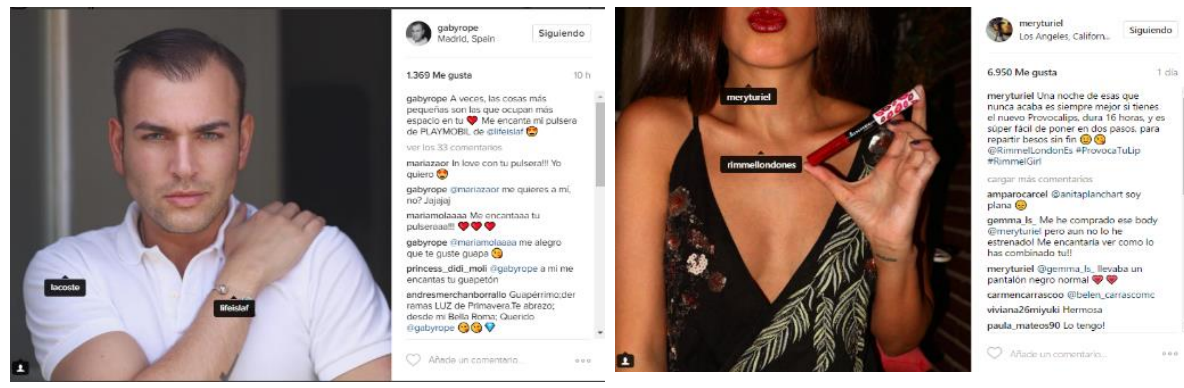

Imagen 4. Mención reiterada a una marca que sugiere una relación profesional entre las partes

El resto de publicaciones -el $20 \%$ para los hombres y el $51 \%$ para las mujeres influencers- tienen como finalidad mantener el vínculo con su comunidad de seguidores mostrando su día a día. Esta circunstancia les sirve de excusa para describir sus outfits y detallar, generalmente a través de etiquetas, las prendas que portan. En esta categoría también hay espacio para los consejos, las recomendaciones o los sorteos: técnica que permite aumentar rápidamente el número de seguidores y la visibilidad alcanzada, ya que, generalmente, requiere a los participantes subir una imagen y etiquetar al producto para poder ser tenido en cuenta.

Visto todo esto, podemos identificar que la mayoría de las entradas publicadas por los influenciadores en su perfil de Instagram tienen un objetivo comercial orientado a promocionar y mejorar la imagen de determinados productos y marcas vinculadas principalmente con el ámbito de la moda y/o la belleza, aunque también se han encontrado ejemplos ligados al ocio, la gastronomía y el turismo. 


\section{MCS - Masculinities and Social Change, 7(3) 249}

\section{Conclusiones}

Con el objetivo de profundizar en la figura del influenciador de moda, esta investigación analiza los recursos comunicativos utilizados por los principales prescriptores españoles de moda en Instagram poniendo una especial atención en el uso que hacen de la imagen y del texto. Asimismo, se indaga en las posibles diferencias entre géneros y en el cumplimiento de la normativa en materia de Publicidad, comprobando si los mensajes incluyen algún elemento que distinga a las publicaciones comerciales -las previamente pactadas-, de las recomendaciones libres y autónomas de sus autores.

La observación minuciosa de las publicaciones de 13 influencers concluye que ninguno especificó de forma explícita disponer de una relación profesional con alguna marca a pesar de identificarse conductas como las siguientes, que si bien no son una prueba inequívoca de cooperación sí representan un indicio: halagos constantes, alusión reiterada a marcas concretas y a sus perfiles en Instagram, y utilización de hashtags que o bien contenían de forma explícita el nombre de la misma marca o bien habían sido creadas por estas siguiendo el eslogan de su última campaña. Se observó que todos estos procedimientos confluían en un mismo mensaje en el $80 \%$ de las publicaciones masculinas, mientras que las influencers femeninas se mostraron más sutiles combinando la intencionalidad más comercial con el cuidado de su comunidad de seguidores -presente en el $51 \%$ de las publicaciones-. Propósito, este último, que se materializó a través de la descripción minuciosa de sus outfits; del ofrecimiento de sugerencias o consejos; o con el anuncio de descuentos y promociones en determinadas marcas.

Y es precisamente esa actitud, la de estar permanentemente preocupadas por ofrecer contenidos útiles a su comunidad de seguidoras, el elemento diferenciador más pronunciado entre los hombres y las mujeres influencers analizadas. Cualidad que queda reafirmada si, además, atendemos a la frecuencia de publicación: ellas se mostraron mucho más prolijas al difundir cerca del doble de mensajes que sus compañeros hombres. La actitud con la que se enfrentan a la cámara también supone un punto de inflexión: ellos miran con descaro y de forma directa al objetivo, con posados evidentes mientras que ellas prefieren transmitir la sensación de haber sido "pilladas" mientras ejecutaban alguna acción de escasa trascendencia. 
A pesar de las diferencias, también se han encontrado conductas coincidentes en ambos géneros relativas: a la preocupación por la estética de las imágenes y su encuadre; al protagonismo indiscutible del influencer y su presencia constante; y a la preferencia por el idioma castellano para expresarse.

Los datos recopilados conducen a concluir que la finalidad predominante de las publicaciones aparecidas en Instagram por los principales influencers de moda en España es la de promocionar: dar a conocer y/o mejorar la imagen, principalmente de prendas, productos o complementos de moda por los que dicen sentir simpatía y afinidad. La duda que sigue quedando en el aire es si, en algún momento las publicaciones estuvieron guiadas por un acuerdo comercial previo, pues como se ha indicado no existe aviso explícito al respecto por parte de estos prescriptores a pesar de los indicios encontrados. Esta situación nos impide asegurar con contundencia que se haya quebrantado la ley, lo cual nos anima aún más a seguir ahondando en el tema.

Además, el gran influjo que las imágenes tienen sobre la sociedad, y más en particular sobre los jóvenes -cuya personalidad y rasgos se están formando- y su capacidad para perpetuar una identidad de género estereotipada demanda una educación de la mirada que empodere al ciudadano de recursos para enfrentarse a los mensajes audiovisuales de forma crítica. Como se ha comprobado con el estudio de las imágenes difundidas por los principales influenciadores de moda en España, hay un afán por mostrar vidas y cuerpos idílicos que como recuerda Lomas y Arconada (2005) son fuente de frustración para la mayoría.

\section{Notas}

${ }^{1}$ El término Iger se utiliza como sinónimo de instagramer y alude a los usuarios más activos de la red social.

${ }^{2}$ Según datos recogidos en abril de 2017

\section{Referencias}

Aswani, R., Ghrera, S.P., Kar, A.K., \& Chandra, S. (2017). Identifying buzz in social media: a hybrid approach using artificial bee colony and knearest neighbors for outlier detection. Social Network Analysis and Mining, 7 (38). doi: https://doi.org/10.1007/s13278-017-0461-2 
Belmonte Arocha, J. (2014). Del arte cinematográfico a la imagen postelevisiva: co-educación audiovisual ante las representaciones de la feminidad. Dossiers Feministes, 19, 149-167. Retrieved from http://www.e-revistes.uji.es/index.php/dossiers/article/view/1452

Belmonte, J. \& Guillamón, S. (2008). Co-educar la mirada contra los estereotipos de género en TV. Revista Comunicar,31,115-120. doi: https://doi.org/10.3916/c31-2008-01-014

Benítez-Eyzaguirre, L. (2016). Analysis of peer recommendations in the online reputation of organizations. El Profesional de la Información, 25 (4), 652-660. doi: https://doi.org/10.3145/epi.2016.jul.15

Bigné, E., Küster, I. \& Hernández, A. (2013). Las redes sociales virtuales y las marcas: influencia del intercambio de experiencias eC2C sobre la actitud de los usuarios hacia la marca. Revista Española de Investigación en Marketing ESIC, 17(2), 7-27. doi: https://doi.org/10.1016/S1138-1442(14)60022-X

Booth, N. \& Matic, J.A. (2011). Mapping and leveraging influencers in social media to shape corporate brand perceptions, Corporate Communications: An International Journal,16(3),184-191. doi: https://doi.org/10.1108/13563281111156853

Castelló, A. (2010). Estrategias empresariales en la web 2.0, las redes sociales online. Alicante: Editorial Club Universitario.

Castelló, A. \& Pino, C. del (2015). Prescriptores, marcas y tuits: el marketing de influencia. Revista Internacional de Investigación en Comunicación aDResearch ESIC, 12(12),86-107. Retrieved from https://rua.ua.es/dspace/bitstream/10045/48350/1/2015_Castello_DelPino_Redmarka.pdf

Castelló, A. \& Pino, C. del (2017). La estrategia publicitaria basada en influencers: el caso de smartgirl by samsung. In A. Castelló Martínez y C. del Pino Romero, (Eds.), Publicidad y Convergencia Mediática. Nuevas estrategias de comunicación persuasiva (pp.116-146). Sevilla, España: Egregius.

Chicharro Merallo, M. (2013). Representaciones de la mujer en la ficción postfeminista: Ally McBeal, Sex and the City y Desperate Housewives. Papers. Revista de Sociología, 98(1),11-31. doi: http://dx.doi.org/10.5565/rev/papers/v98n1.469 
Ferreiro Habra, A. (2018). Masculinidades en el discurso publicitario. Question, 1(58), e053. https://doi.org/10.24215/16696581e053

Fresno, M. del., Daly, A. J., \& Segado, S. (2016). Identificando a los nuevos influyentes en tiempos de Internet: medios sociales y análisis de redes sociales. Revista Española de Investigaciones Sociológicas, 153, 2342. doi: $10.5477 /$ cis/reis. 153.23

Gillin, P. (2009). Los nuevos influyentes. Madrid: LID Editorial.

González-Romo, Z., García-Medina, I. \& Macdonald, L. (2016). Bloggers cómo influenciadores de consumo, referencia y opinión en el sector de productos de cosmética femenina. Revista Internacional de Investigación e Innovación Tecnológica, 4 (20), 1-12. Retrieved from http://riiit.com.mx/apps/site/files/bloggers_cmo_influenciadores_de_c onsumo_referencia_y_opinin_en_el_sector_de_productos_de_cosmtic a_femenina.pdf

IAB SPAIN. Interactive Advertising Bureau Spain (2015). VI Estudio anual de redes sociales. Madrid: IAB Spain. Retrieved from http://www.iabspain.net/wpcontent/.../2015/01/Estudio_Anual_Redes_Sociales_2015.pdf

IAB SPAIN. Interactive Advertising Bureau Spain (2016). Estudio anual de redes sociales. Madrid: IAB Spain. Retrieved from http://www.iabspain.es/wp-

content/uploads/iab_estudioredessociales_2017_vreducida.pdf

Krippendorff, K. (2004). Content Analysis: An Introduction to Its Methodology. California: SAGE.

Launchmetrics (2017). Informe sobre el estatus del marketing de Influencers 2017. Retrieved from

https://www.launchmetrics.com/es/recursos/whitepapers/informemarketing-influencers-2017

Ley 3/1991, de 10 de enero, de Competencia Desleal, BOE núm. 10, pp. 959962.

Ley 34/1988, de 15 de noviembre, General de Publicidad, BOE núm. 274, pp. $32464-32467$.

Lomas, C. (2005). ¿El otoño del patricarcado? El aprendizaje de la masculinidad y de la feminidad en la cultura de masas y la igualdad entre hombres y mujeres. Cuadernos de Trabajo Social, 18, 259-278. Retrieved

from 
http://revistas.ucm.es/index.php/CUTS/article/view/CUTS050511025 9A/7596

Lomas, C., \& Arconada, M. Á. (2003). La construcción de la masculinidad en el lenguaje y en la publicidad. En C. Lomas (comp.), ¿Todos los hombres son iguales? Identidades masculinas y cambios sociales (pp. 145-201). Barcelona: Paidós.

Martínez, E. \& Gaona, C. (2016). Límites jurídicos de la publicidad en redes sociales: Facebook, Instagram y Twitter. In C. Mateos Martín \& F. J. Herrero Gutiérrez (Eds.), La Pantalla Insomne (pp.2410-2421). Santa Cruz de Tenerife, Spain: Cuadernos artesanos de Comunicación.

Martínez-Sanz, R. (2013). El blog a las puertas del museo, pero ¿por qué no entra? El caso de los centros de arte contemporáneo en España. Revista Telos. Cuadernos de Comunicación e Innovación, 95, 108-115. Retrieved from http://eprints.rclis.org/20266/2/pdf-generator.pdf

McCormick, K. (2016). Celebrity endorsements: influence of a productendorser match on Millennials attitudes and purchase intentions. Journal of Retailing and Consumer Services, 32, 39-45. doi: https://doi.org/10.1016/j.jretconser.2016.05.012

Pérez, C., Clavijo, L., Luque, S. \& Pedroni, M. (2017). Social Media y Comunicación Corporativa. Nuevo reto en las empresas de Moda. In J. Herrero (Coord.), Del verbo al bit (pp. 2029-2059). La Laguna, Spain: Sociedad Latina de Comunicación Social.

Ribeiro-Cardoso, P., Teixeira, S., \& Santos, A. N. (2016). Liderazgo de opinión de moda, adhesión a la innovación y actitud hacia la publicidad entre los consumidores portugueses. Revista Mediterránea de Comunicación, 7(2), 101-115.

https://doi.org/10.14198/MEDCOM2016.7.2.12

Rojas, P. \& Redondo, M. (2017). Cómo monetizar las redes sociales. Madrid: LID Editorial.

Ruiz Cartagena, J. J. (2017). Millennials y redes sociales: estrategias para una comunicación de marca efectiva. Revista Miguel Hernández Communication Journal, 12(8), 347-367.

http://dx.doi.org/10.21134/mhcj.v0i8.196

Sartori, G. (1998). Homo videns: la sociedad teledirigida. Madrid: Taurus. 
Uzunoḡlu, E. \& Kip, S. (2014). Brand communication through digital influencers: Leveraging blogger engagement. International Journal of Information Management, 34 (5), 592-602. doi :

https://doi.org/10.1016/j.ijinfomgt.2014.04.007

Venegas, A. (2015). Marcas vs. "Influencers": matrimonio de conveniencia. Agencias y "Blogueras" ofrecen sus puntos de vista sobre el estado actual del marketing de influencia. Revista Anuncios: Semanario de publicidad y marketing, 1516, 20-24. Retrieved from https://dialnet.unirioja.es/servlet/articulo?codigo $=5234474$

Wiedmann, K.P., Hennings, N. \& Langner, S. (2010). Spreading the word of fashion: Identifying social influencers in fashion marketing. Journal of Global Fashion Marketing: Bridging Fashion and Marketing, 1(3), 142-153. doi: https://doi.org/10.1080/20932685.2010.10593066

Raquel Martínez-Sanz es profesora Ayudante Doctor en el Departamento de Historia Moderna, Contemporánea y de América, Periodismo y Comunicación Audiovisual y Publicidad de la Universidad de Valladolid, España

Cristina González Fernández es periodista especializada en moda, España

Dirección de contacto: Correspondencia directa a Raquel Martínez-Sanz, Facultad de Filosofía y Letras. Plaza del Campus, s/n, 47011 Valladolid, España, email: raquel.martinez.sanz@uva.es 


\section{Hipatia Press}

Instructions for authors, subscriptions and further details:

\section{Production of Gender: A Study on Performativity in Female-To- Male Transsexuals}

Tanupriya \& Dhishna Pannikot ${ }^{1}$

1) National Institute of Technology Karnataka, India

Date of publication: October $21^{\text {st }}, 2018$

Edition period: October 2018 - February 2019

To cite this article: Tanupriya, \& Pannikot, D. (2018). Production of Gender: A Study on Performativity in Female-To-Male Transsexuals. Masculinities and Social Change,7(3),255-278. doi:

10.17583/MCS.2018.3607

To link this article: http://doi.org/10.17583/MCS.2018.3607

PLEASE SCROLL DOWN FOR ARTICLE

The terms and conditions of use are related to the Open Journal System and to Creative Commons Attribution License (CC-BY). 
pp. 255-278

\title{
Production of Gender: A Study on Performativity in Female-To-Male
}

\section{Transsexuals}

\author{
Tanupriya \& Dhishna Pannikot \\ National Institute of Technology Karnataka, India
}

\begin{abstract}
Gender identity is critical to every individual; it is self-defined and constructed by culture and society, at large. Gender identities are formed through public and private spaces. Of the two traditions of thinking (essentialist and constructionist) about sex and gender, constructionist formulations are based on 'performance' theory. It believes that sex and gender are viewed as not residing in an individual, but are found in interactions that are socially constructed as gendered, as opposed to essentialist tradition. Within gender theory, 'performativity' of gender can be traced from Beauvoir's (1949) construction "one is not born a woman, but rather, becomes one" (p.8) suggests the constructed and performative nature of gender. Butler (1999) in her theory of gender performativity also emphasizes on the constructed nature of sex and gender, and refers gender as "the repeated stylization of the body, a set of repeated acts within highly rigid regulatory frame" (p.33). Within 'performative' theory, gender is a process, rather than something naturally possessed. To understand and explore the process of formation of gender in (FTM) transsexuals three autobiographical narratives are chosen i.e., Becoming a Visible Man (2004), The Testosterone Files (2006), and Both Sides now (2006). To analyze and dissect the FTM autobiographical narratives, the techniques of discourse analysis, literary analysis and narrative analysis are used. This study will address the performative factors that contribute to the formation of a transsexual role as seen through sartorial style, mannerisms, and other aspects that influence a gendered presentation of 'self'. It includes the process of construction of FTM transsexual's corporeality through performative attributes to approximate masculinity and come in accord with the social role of a 'man'.
\end{abstract}

Keywords: body, gender, FTM, transsexuals, gender identity. 


\title{
Producción de Género: Un Estudio
}

\section{sobre la Performatividad de Mujeres a}

Hombres Transexuales

\author{
Tanupriya \& Dhishna Pannikot \\ National Institute of Technology Karnataka, India
}

\section{Resumen}

La identidad de género es crítica para cada individuo; es una realidad autodefinida y construida por la cultura y la sociedad. Las identidades de género se forman a través de espacios públicos y privados. Existen dos tradiciones de pensamiento principales (esencialistas y construccionistas) sobre el sexo y el género, las formulaciones construccionistas se basan en la teoría del "desempeño". En contraposición a la tradición esencialista, el construccionismo cree que el sexo y el género no residen en un individuo sino que se encuentran en las interacciones que se definen socialmente. Dentro de la teoría de género, la "performatividad" se puede profundizar a partir de la construcción de Beauvoir (1949) "una persona no nace mujer, sino que se convierte en una persona" (p.8). La autora sugiere la naturaleza construida y performativa del género. Butler (1999) en su teoría de la performatividad de género también enfatiza la naturaleza construida del sexo y el género, y refiere al género como "la estilización repetida del cuerpo, un conjunto de actos repetidos dentro de un marco regulatorio altamente rígido" (p.33). Dentro de la teoría "performativa", el género es un proceso, más que algo naturalmente poseído. En el presente artículo, para comprender y explorar el proceso de formación del género en personas transexuales (FTM) se eligen tres narrativas autobiográficas, es decir, Becoming a visible man (2004), The testosterone files (2006) y Both sides now (2006). Para analizar y diseccionar las narrativas autobiográficas FTM, se utilizan las técnicas de análisis del discurso, análisis literario y análisis narrativo. Este estudio abordará los factores performativos que contribuyen a la formación de un papel transexual, en concreto a través del estilo de vestimenta, manierismos y otros aspectos que influyen en una presentación del "yo" de género. Ésto incluye el proceso de construcción de la corporeidad de las personas transexuales de FTM a través de atributos performativos que se aproximen a la masculinidad y el seguimiento del rol social de "hombre".

Palabras clave: cuerpo, género, FTM, transexual, identidad de género. 


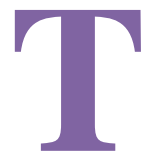

he term "transsexual" was coined and publicized in the early 1950s by Harry Benjamin and David Cauldwell when news about Christine Jorgenson's sex change, through surgery and hormones, triggered in American newspapers. Jorgenson's sex transformation was highly publicized by the American media even when Meyerowitz (1998) states that, news about 'sex change' and 'sex transformation' had appeared in newspapers and magazines since the 1930s. The concept of sex change and sex transformation existed much before the term "transsexual" was executed in medical discourses. The early research on sex transformation traces back to European origins and research by European sexologists such as Magnus Hirschfeld, who defined and coined the term 'transvestism', and Havelock Ellis who defined 'eonism' as a separate category that included cross gender identification and cross dressing. 'Transsexualism' did not appear as a medical parlance until early 1950s. The term 'transsexual' was popularized only after the Jorgenson's sex change surgery which was a widely read and published headline during that time. The publication of Jorgenson's autobiography Christine Jorgenson: A Personal Autobiography in 1967, came as a reply to a storm of hysterical press coverage about her sex change. Jorgenson's autobiography was a turning point for transsexuals and Hausman (2006) mentions the impact of her autobiography on transsexuals. Mario Martino, in his autobiography Emergence, mentions that, "as Marie, she was the first in her town to buy Christine Jorgenson's autobiography when it came out in 1967' (p.336). The autobiography in a way validated their desire and helped to identify themselves as transsexuals (transman or transwoman), hence allowing individuals to come in terms with their identity, and construct their identity as transsexuals. Conundrum (1974) by Jan Morris, appeared a decade later after Jorgenson's autobiography. In her autobiography, Morris credits Jorgenson with the liberalization of public attitude towards medical treatment of transsexuality. In 1977, Mario Martino published Emergence: A Transsexual Autobiography, the first female-to-male autobiography but it didn't gain much readership and popularity like Jorgenson's and Morris's autobiography. Perhaps, the possible reason could be, as Califia (1997) states, "transsexuality was no longer such a shocking idea or because public had much less interest in the phenomenon of biological females becoming men" (p.38) as the "gender 
transition from female to male allows biological women to access male privilege within their reassigned genders" (Halberstam, p.143). Theorists Judith Halberstam and Jay Prosser, in their works Female Masculinity (1998) and Second Skins (1998) provide an extensive analysis of FTM autobiographical narratives. Their perspective to approach FTM transsexuality is different, as Halberstam understands FTM transsexuality from the perspective of a lesbian feminist, whereas Prosser establishes FTM transsexuality from an FTM transsexual perspective. To interpret and provide clear differences between identities Halberstam (1998) formulates a masculine continuum based on lesbian and transgender masculinities i.e., "Androgyny- Soft Butch- Butch- Strong Butch- Transgender Butch- FTM not Masculine- Very Masculine” (p.151). Martino's autobiography provides differences between butch and FTM transsexual, as to disentangle his identity as an FTM transsexual from butch. Mario explains, "A butch is the masculine member of the lesbian team" (p.141). Another transsexual autobiography Dear Sir or Madam (1996) by Mark Rees also magnifies the gulf between butch and transsexual male to mark out the boundaries of transsexual masculinity. The autobiographies can be considered as a way through which transsexuals communicate and share their experiences.

The earlier published autobiographical accounts of female-to-male autobiographies can be roughly traced as Mario Martino's Emergence: A Transsexual Autobiography (1977), Raymond Thompson and Kitty Sewell's What Took You So Long (Memoir) (1995), Mark Rees's Dear Sir or Ma'am (1996), Jamison Green's Becoming a Visible Man (2004), Matt Kailey's Just Add Hormones (2005), Max W. Valerio's The Testosterone Files (2006), Rico Adrian Paris' Transman - Bitesize: The Story of a Woman Who Became a Man (2005), Dhillon Khosla Both Sides Now (2006), and Thomas Beatie's The Story of One Man's Extraordinary Pregnancy (2008). Transsexual autobiographies marked the establishment of an official discourse regulating the self- representation and provided an orientation to other individuals who identified themselves as transsexual after reading the autobiographies. Earlier, to undergo sex reassignment surgery, clinicians or the medical practitioners, demanded a personal history before carrying out the operation, and the success in obtaining treatment depended on "their ability to convince doctors that their personal history matches the officially sanctioned etiology" (Bolin, 1988, p.64; 
Green, 1987, p.7-8). So, the published accounts became the "guide-books" (Hausman, 2006, p.337) for transsexuals to obtain desired medical treatment. But, transsexual autobiographies cannot be just considered as the part of repressive structure of "official" (Hausman, 2006, p.339) transsexual experience, as they enable others to form a transsexual identity as transsexuals. Bernice L. Hausman in Body Technology and Gender in Transsexual Autobiographies (2006) situates the importance of transsexual autobiographies and shows the effect of writing autobiography on transsexuals. He mentions;

Mario Martino writes in Emergence that, as Marie, she was the first in her town to buy Christine Jorgenson's autobiography when it came out in 1967. In her autobiography Conundrum, Jan Morris discusses the emotional significance of finding Lili Elbe's autobiography. Nancy Hunt writes in Mirror Image, "I can remember only once when my life has been altered by the printed word (p.336).

This study attempts to understand the performative aspect of sexuality in transsexuals, as Bolin (1988) and Stone (1991) suggests that, "in transsexual stories, the truth of the transsexual experience, are about sexuality" (p.339). Foucault (1977) also stresses on the ways in which sexuality is written in or on the 'body', he deconstructs the notion that sexuality is a transparent face of life. Purvis in his essay "Sexualities" (2006) states, "If sexuality is inscribed in or on the body, then it is texts and discourses (literary, medical, legal and religious, for example) which make the sexual into something that is also textual" (p.435). To argue, sexuality and textuality are linked, is to propose that sexual is conceived in relation to words, sign systems, discourses and representations" (p.436). It is within and against the careful reading of texts that sexualities can be re-written or re-conceived. Jay Prosser also explores trans narratives in his work Second Skins: The Body Narratives of Transsexuality (1998) which examines the literary texts that discuss themes of transitioning and discusses the importance of studying trans narratives.

The theory of 'performativity' of gender by Butler can be applied for both transmen and transwomen, as the theory isn't indicative of any identity, but describes the performative nature of gender. Often, in 
discourses about transmen and transwomen, it is seen that transmen have had less visibility than other forms of gender variance and thus it occupies a very less space in trans discourses. For instance, In India, there are no published accounts of transmen autobiographies, which provides a very narrow space about discussing their identity. Beasley (2005) mentions that masculinities only focus on cisgender masculinities and thereby reinforcing certain constructions of masculinity and side-lining the masculinities of a plurality of gender non-conforming men. Aboim (2017) mentions, "transmen have also received less attention from the part of 'Trans Studies' when compared to their female counterparts, which have gained far more visibility" (p.226). Literature explicitly focuses on the experiences of transmen that emerged in the late 1990s and early 2000s. These studies include the one by Jamison Green (2004), studies by Aaron Devor (1997), Henry Rubin (2003), Jason Cromwell (1999) and Stephen Whittle (2002).

The female-to-male autobiographies chosen for this study are Jamison Green's Becoming A Visible Man, Max W. Valerio's The Testosterone Files and Dhillon Khosla's Both Sides Now. These narratives could be considered as contemporary transsexual narratives and autobiographical narrative of Jorgenson, Rees, Martino and Thompson, can be considered as first wave of autobiographies, that are also analyzed by theorists such as Jay Prosser, Bernice Hausman and Judith Halberstam. These autobiographies are responsible for the formation and setting up of the discourses and can be considered as classics in the context of transsexual autobiographies. The autobiographical narratives selected for the study were chosen after a careful reading of other autobiographical narratives too. Few commonalities have been traced between these three autobiographies. The first being that these autobiographies were published in the $21^{\text {st }}$ century and showed a linear pattern of transition. Jonathan Ames in Sexual Metamorphosis: An Anthology of Transsexual Memoirs (2005), compares transsexual autobiographies (of both FTM and MTF trans people) to a classic literary model, the bildungsroman, or coming-of-age novel. He states that he sees a basic outline of transsexual memoirs in three acts, "first act: genderdysphoria childhood; second act: the move to the big city and the transformation... [third act] the sex change" (p.xii). Within these three autobiographies, there is a linear story of transitioning, where the authors track their transition in a linear fashion. From identifying themselves as 
lesbian in the beginning to finding their 'self' as transmen. Jay Prosser's Second Skins: The Body Narratives of Transsexuality (1998) explores transsexual autobiographies in the chapter entitled "Mirror Images: Transsexuality and Autobiography", and he examines the way autobiography is an important process where "the trajectories of transsexuality and autobiography are entwined in complex ways, with narrative and bodily form, conducting each other" (p.101). The portrayal of 'self' and identity formation are explored vividly in the transsexual autobiography.

Research studies in literature are conducted applying many innovative methods. The methods employed for this study are literary analysis, discourse analysis, and narrative analysis. Through various gender theories, the transsexual autobiographies are considered as open to interpretations. Ekins and King in The Transgender Phenomenon (2006), talks about the process of Migrating, Oscillating, Negating and Transcending as part of the formation of their identity. The autobiographies can be analyzed through various lens, but for this study, it will investigate the functioning of performativity as a part of their sexuality in the life of transsexuals.

Discourse analysis is another important research method employed in this study. Discourse analysis is concerned with producing an analysis or 'explanatory critique' (Fairclough, p.235-236) of how and to what purpose language use is invested through deployment of specific textual features to facilitate the knowledge of its effects. The term 'discourse' itself means multiple meanings, like the Derridean notion of 'deconstruction' where every signifier refers to other words/signifier in an 'endless postponement of meaning'. Hence, one travels along the path of meaning making. Yet another method i.e., 'narrative inquiry' uses autobiography and life experience, as the units of analysis to research and understand the way people create meaning in their lives, as narratives. To carry out narrative analysis in qualitative studies, Creswell (2007) provides four elements to describe, classify and interpret the text i.e., codes, categories, patterns and themes. Codes involves identifying concepts from the text, categories include, linking codes to create a unit or a category, themes involves creating a theme that represents similar patterns, and patterns are identifying repeated units from categories. So, discourse analysis, narrative 
analysis and literary analysis are used as tool to interpret and analyze the texts.

\section{Aspects of Performativity}

Beauvoir makes a point that "one becomes a woman, but always under a cultural compulsion to become one" (Butler, 1999, p.8). This statement implies that the sexed body may or may not come in terms with the gender role assigned. Body thus, is a surface on which various performative acts can be carried out, to affirm one's gender identity. But Gender theorist, Butler makes it clear in her work Gender Trouble that, "performativity must be understood not as a singular act, but rather as the reiterative and citational practice by which discourse produces the effects that it names" ( $p$. xii). Thus, gender identity is a result of repeated acts, and through performative attributes transsexuals claim their authority over their desired gender identity.

Masculinity in a female body is enacted not only through surgeries and hormones but through a correlation of 'performativity', 'representation' and 'transition'. It is a process of 'becoming a man' and trying to ascertain a male identity and masculine presentation of self. Beynon (2002) mentions, "Men are not born with masculinity as part of their genetic make-up; rather it is something into which they are acculturated and is composed of social codes of behavior which they learn to reproduce in culturally appropriate ways" (p.2). This observation describes the performative nature of gender. Femininity and masculinity are both culturally shaped and can be experienced and enacted. According to Butler (1999), gender identity does not cause gendered behavior, but rather performing gender creates the sense of an internal gender identity. In order to create an identity that is approximately masculine, FTM transsexuals undergoes surgeries and adapts masculine attributes. It includes behavior that is primarily masculine, which includes imitating the male sartorial style, their desire for women, "passing" as man, using cosmetics to draw moustaches, and relate themselves with male experiences and desires.

Theorist Jay Prosser (2006) suggests from a reading of Butler's text that transsexualism is equally productive and not essentialist. It was argued by Prosser that, even though Butler's Gender Trouble (1999) discusses performativity in the final chapter "Bodily Inscriptions and Performative 
Subversions", it is gender "performativity" that Gender Trouble is known for. While it argues that all gender is 'performative', that man and woman are not expressions of prior internal essences, but is constituted, "through the repetition of culturally intelligible stylized acts" (p.33). Gender Trouble presents the transgendered subject as the concrete example that brings into relief 'performativity' of gender (p.31). In Bodies that Matter (1993) Butler explains performativity as;

Performativity is thus not a singular act, for it is always a reiteration of a norm or set of norms, and to the extent that it acquires an act like status in the present, it conceals or dissimulates the conventions of which it is a repetition (p.13-14).

According to Butler, all performances that are understood as reflections of an essential identity or 'self' are 'constative performances'. By 'constative', she means those performances of identity that actively construct the identity, they are taken to be expressions of.

Butler (1999) mentions that body, sex and gender are performative categories. She mentions that gendered body is performative, and it has no prior ontological status, apart multiple and repeated acts that constitute its reality (p.136). Butler (1999) emphasizes on the constructed nature of sex and gender. She deconstructs the innate basis of one's gender identity and considers gender as 'performative', which can essentially come into expression through repetition of acts, utterances, habits, and gestures. It expresses that gendered identities are actually "fabrications manufactured and sustained through corporeal signs and other discursive means" (p.136). Repetition and recitation establish some of these utterances as 'proper', but they are not fixed. In similar lines, Khosla (2006) in his autobiographical narrative, repetitively mentions about his practice of fixing a fake phallus to feel a complete man. "I grabbed a fresh pair of underwear and tucked in my prosthesis, pulling it up tight against my body" (p.171). Recitation establishes the ground for identity formation and it is a discursive process. Performativity is thus, not about the singular act, but plural acts and the discursive process of configuring the body that presents individuals as male or female, masculine and feminine. In this sense, masculinity is not seen in an essentialist perspective for men who possess physiologies and corporeality. Masculinity is seen as a free-floating artifice, which isn't 
exclusive for males, but includes female experiences of being a male or a man. Female masculinity has not only shattered the essentialist and fixed domains of masculinity, it has also portrayed that even if individuals are bound to social roles of man and woman, and gender identity is what distinguishes them from one another.

\section{Production of Gender}

Production of gender means the process of producing gender through a set of repeated stylized acts. Butler's (1999) concept of 'corporeal signification sees body as a surface on which acts, gestures, desires and performative attributes are carried out which results in the production of identity which is close to natural being (p.173). Bristow (1997) also sees body as a platform which give gendered connotations to acts, construction and performative attributes. It is through sartorial style, 'passing' and 'performative' acts that one constructs an identity as a transsexual. The first literature on transsexuals have attributed transsexuals' specific characteristics, behaviors, identities, and sexualities. Individuals were expected to fit within these attributions, which became the diagnostic criteria and were considered the etiological factors in the diagnosis of "true" transsexualism (Stoller, 1975, p.74). After a thorough reading of autobiographies, select factors such as, 'passing' as a man, the concept of wrong body, transition from sex to sexuality, trajectories of transsexuals, surgical procedures and erasing femininity are the common themes found in select autobiographies. The body is the site on which individuals "erect a reliable sense of self" (Boddy, 1995, p.135). The themes discussed below are an attempt to understand the sexuality of transsexuals, and body being one of the aspects to enhance masculinity in a female body. The study traces the performative attributes and processes adopted by authors to gain the sense of a male identity. The theoretical concepts such 'passing', 'wrong body', 'trajectories of transsexuals', 'etiological construction of FTMs' are considered as the performative elements to describe and understand the construction of a transsexual body. These concepts can be applied in the cases of transwoman too. 


\section{'The Concept of 'Passing': 'Passing' as a 'Man'}

'Passing' signifies performance and passing as a man is an important factor which influence transsexual lives and construct a male social role. The concept of 'passing' dates back to eighteenth century and its original usage as Friedli (1987) who used it the context of African Americans 'passing' as whites (p.251). 'Passing' is perceived as a performance and the concept of passing is associated with transgenders and transsexuals. In the context of transsexual discourses, 'passing' means blending in and becoming unnoticeable and unremarkable, as either a man or a woman. 'Passing' is the notion that a person dresses in the clothing of the opposite dress "for convincing an 'unknowing audience' that one actually is a member of that sex" (p.179) as Anne Herrmann defines in "Passing Women, Performing Men" (1991). Stoller (1965) mentions that "passing is problematic for trans people and is related to the problems of identity formation" (p.191). Similarly, Weigert et al (1986), mentions, "the predicament of the transsexual, specially the preoperative one, provides a rich example of how an individual engages in artful impression management to present a problematic gender identity as beyond doubt and within a totally taken-forgranted world of social action" (p.75). 'Passing' within this discourse is only problematic, when artful impression management is not successful, and the person is read (e.g., as a man in a dress or as a woman in a man's suit). One of the important factors when it comes to passing is the use of the female pronouns for FTM transsexuals.

In Both Sides Now, Khosla (2006) gave a lot of thought to the process of passing and in many instances, he was seen contemplating about his looks. "I remember feeling deep gratitude toward him, followed by sadness. He was calling me 'he' because he had read the memo, not because I looked like it" (p.64). The constant fear to be referred as female is evident from the autobiographies. Green (2004) puts the concept of 'passing' as a man not limited to bodily modifications, but to the self. He questions the implicit connotations linked with the category 'man' and dismisses it. Green mentions;

What makes a man a man? His penis? His beard? His receding hairline? His lack of breasts? His sense of himself as a man? Some men have no beard, some have no penis, some never lose their hair, 
some have breasts; all have a sense of themselves as men. Transsexual men are also men. Transsexual men are men who have lived in female bodies (p.186-187).

Green (2004) understands 'passing' as a 'performance' and sees "changing sex as just one way of changing one's body" (p.89), he also questions, "so why change one's body? Because the body is where we live and through it we communicate to others" (p.95). Green also describes the importance of and the concept of passing as a man, in the life of all men, trans or non-trans. Green describes the very nature of gender roles and gender identity and thus, confirms that the concept of passing as a man or woman is crucial for transsexuals and cisgenders both. He mentions;

The crux of the matter of gender for anyone is their own visibility and enough external confirmation of their gender identity; thus, if a person is comfortable with her or his gender-body congruity or incongruity and their gender identity is confirmed by people around them whom they value, they will feel seen and validated by others (p.187).

From this observation, the importance and functioning of performativity in everyday life of transsexuals and cis- males is emphasized. Raewyn Connell (2012) mentions that "most of the discussions about transsexuality are focused on identity questions" (p.863). He also mentions that bodies form the vital arena of contradiction and change. After changing one's body, the process and struggle of passing comes into picture. Khosla's autobiographical (2006) account greatly focused on his struggles of passing as a man, looking manly enough, and the inexplicable pain he feels when referred with feminine pronouns. He mentions, "I don't think I could ever fully describe the pain and anguish of hearing "she" and "ma'am" and "miss" (p.54). He describes an incident after his mastectomy, when he was excited that he finally looks like a man, but after being referred to as "ma'am" (p.56) in a restaurant, he mentions, "it hit me like a sucker punchlike everything I had just gone through had been ripped out from under me in one fell swoop" (p.56). He described his anguish and failing to pass as a man, "How am I supposed to have people call me 'he' at work when I still 
look the same?" (p.57). In one of the instances, to pass as a male, Khosla used his old kajal to draw moustache and beard on his face. He mentions;

\begin{abstract}
I had asked my therapist whether she knew specifically how hormones changed the face. Did the face shape change or was it just facial hair that made one look male? She answered that she thought it was just the facial hair and then I could specific things to make it easier to "pass" as male, such as cutting my hair even shorter and removing my one earring (p.36).
\end{abstract}

The concept of passing is applicable to transsexuals in general even with the change in the culture or region. Passing as woman or man, establishes them as a gender identity and social role of a woman or man. Thus, the very nature of citing, reiterating, reenacting a performative act establishes a gender identity.

\title{
Anxiety of being in a Wrong Body
}

Critic Talia Bettcher's (2014) idea of 'wrong body' in transsexuality involves a misalignment between gender identity and the sexed body. The phrase 'wrong body' describes the feeling that one's body is not a part of one's self. The insider within the body does not recognize the outside of the body as belonging. The wrong body is envisioned as a state in which body and gender identity do not match, hence "a disparity between body (materiality) and self (subjectivity) is embodied, entertaining dichotomous disjunctions such as the body and its expression, the body and its perception, the body and surrounding gender norms, and sex and gender, which implicitly places sex with (material) genitalia and gender with its (social) expression" (Engdahl, 2014, p.268). Khosla's autobiography (2006) repeatedly mentions the anxiety of being trapped in a female body and his continuing questions to the surgeons before any surgery which describe his incessant desire of a male identity. He would question, "How long it would take for the changes to hold- for me to look like a man" (p.60), "how much more surgery do I need to feel complete"? (p.61). Money's (1990) claim that the concept of being trapped in the wrong body was "adopted by transsexuals as their own" (p. xiv), the idea has been forced on transsexuals by those who have control and have access to medical technologies, and 
have controlled discourses about transsexuals. Cromwell (1999) mentions from the excerpts of a personal communication with a transsexual, he says, "When a man is a man in every way (except) the lower part of the body, he is trapped, and I mean trapped, in a woman's body" (p.104). For many transsexuals, after the surgical procedures and the alteration of body, they no longer consider themselves to be transsexual. Their 'wrong body', now altered becomes a gendered body of woman or man. Valerio's (2006) account presents positive experiences after transition, and he mentions, "If I am out with a woman friend for dinner, I get attention of the waiter or bartender more easily than she does. They look at me for direction and act as though they expect me to pay" (p.187). He describes it as an authority or the ability to move and change situations in the world after his transition and the change in the sex roles. In the case of Valerio (2006), he mentions, "The tits are the first thing I have to take care of. Can't go around with those things hanging out, bouncing all over the place like wild rubber balls. They will definitely put a dent in my male image" (p.128). FTM transsexuals deploy few such practices and procedures to pass as a man and to bring into action the archetype of a complete man. In many instances, these narratives illustrate hatred of, and dissociation from, aspects of their bodies. Transmen used negative expressions to articulate their anger and frustration for having linked with the parts of their bodies which are considered as social markers of female sex. Although this was not an unexpected finding, it was expressed by intensified language to articulate their anger and frustration. Khosla (2006) underwent mastectomy twice to get a flat chest. He mentions, "There was a slight throbbing in my chest and I went to touch the bandages to make sure it was all flat" (p.48). In his autobiography, Khosla gets irritated when he had bandages and was tubed after his surgery and he gets his periods. He states that;

It was not just physically uncomfortable-weakly stumbling to the bathroom to insert a tampon with this contraption of bandages and tubes dangling from my chest. It was psychologically jarring. Here I was in most tentative stage of developing my male body only to be shocked back into reality of my female one (p.51). 
The autobiographical narratives illustrate and reveal the excruciating and harrowing disconnection that transmen undergo due to the incongruity between their bodies and gender identifications.

\section{Trajectories of Transsexuals}

Henry Rubin (2008) mentions, the process of consolidating a transsexual identity as "transsexual trajectory" (p.114). The concept of 'trajectory' is borrowed from sociologist Barbara Ponse, (1978), who develops Goffman's (1959) notion of 'career'. Goffman mentions;

The term is used in a broadened sense to refer to any social strand of any person's course through life... [t] he regular sequence of changes that career entails in the person's self and in his framework of imagery of judging himself and others (p.127-28).

In Ponse's schema, a "trajectory is a non-sequential route to an identity with five stops": (1) experiencing a subjective feeling of difference, (2) finding the appropriate category and assigning the feeling of difference in relationship to that category, (3) accepting the category as descriptive of one's experience, (4) seeking a community, and (5) engaging in relationships. This trajectory holds true for FTMs. Valerio (2006) experienced that subjective feeling of difference from the very beginning and was even referred as a "real tomboy" (p.42). He then identified himself as a lesbian and was linked to the lesbian community for a very short period. But later, he discovered his male 'self' and identified himself as a transman. He describes his instances from childhood and mentions, "I have a very difficult time relating to girls. I'm tormented and teased by a group of girls because I'm unable to play house to their expectations. They try to get me to play their girl games, sweeping floors and wearing fake curlers" (p.43). Valerio's autobiography offers very interesting accounts which portrays the importance of performativity in the life of FTMs. Before getting a mastectomy surgery, he was suggested by fellow FTM transsexual, to use a wide stretch of elastic to his chest to flatten his chest. He mentions, "A method to reel my breasts in, tie them down, flatten my chest with least amount of discomfort" (p.128). Further, the repeated act to flatten his chest through "posture belt" (p.120) describes various aspects of 
performativity. "I've been binding myself flat for six months now" (p.132). Apart from hormones and surgeries, these are practices used by the FTMs to establish an identity that is essentially masculine. David W. Krueger (1983) mentions that "absence of a penis is loathsome" for an FTM (p.77) and "goal is the attainment of a functional penis", be it an artificial penis (p.521) as mentioned by Pauly (1974). Khosla (2006) mentions,

While I had often dreamed of having a penis, I had never given any thought to having testicles. After finally getting rid of my sagging, fleshy breasts, the last thing I had on my mind was adding another set of pendulous sacs of skin" (p.70).

He orders for himself a prosthetic phallus to feel a complete man. Valerio (2006) mentions, "I've been stuffing my briefs with a sock. It works. I safety-pin a large, rolled up athletic tube sock to the inside of my underwear each morning. Position it so that the bulge looks realistic" (p.137). For Jamison Green, bodily modifications are not the sole basis for the construction of gender identity, but on the contrary, he also examines the importance of "corporeality in producing the experience of particular gender consciousness, and complicates the relationship between the social, bodies and performativity" (Raj, 2001, p.2). On the other hand, Valerio's chapter "Patriarchal Underwear Cannibals" (2006) describes the dilemma of an FTM in choosing underwear to wear. He mentions the instances of various transmen, who have always worn men's underwear from the beginning. But Valerio and other transmen were too embarrassed to go and buy a pair of boxer or briefs, describes the subjective experience that transmen go through pre-transition. It is important to note, that each transman have their set of discourses and subjective experiences and masculinity is performed differently on FTM bodies.

\section{Transition}

Hausman (1999) mentions that, body is a visible signifier and thus it is "a mirror of identity" (p.191). She historicizes the relationship between the discovery and synthetization of the sex hormones, gender reassignment surgeries and the theories of gender identity. Masculinity and femininity are binary oppositions formed through dichotomous sexed bodies. Bodies are 
made to go through the gendering discourses, but bodies perform and enact according to their sexuality. Halberstam (1998) asserts that masculinity must not be considered as a constructed or fixed identity to males, but female masculinity should also be accepted. It is not the feeling of a male identity that compels transmen to transition to visible manhood, but the inability to fight society's non-acceptance of female masculinity or "bodies that fail to integrate" (p.147). Halberstam (1998) also perceived that:

...sexual and gender identities involve some degree of movement (not free-flowing but much scripted) between bodies, desires, transgressions, and conformities; we do not necessarily shuttle back and forth between sexual roles and practices at will, but we do tend to adjust, accommodate, change, reverse, slide and move in gender between moods and modes of desire (p.147).

For Green, Valerio and Khosla, the dissonance between masculinity and the female body resulted in a desire to undergo sex reassignment surgery. Bodily change became a necessary factor to 'find' his masculine core due to the parental pressure and romantic frustrations. He describes transitioning, as "to seek an internal sense of comfort" and "to facilitate our being perceived socially by others as the men or women we know ourselves to be" (Green, 2004, p.90). Green (2004) mentions the phase of transition as,

There is also the inevitable fascination with our physical body as it changes right before our eyes into something to which we finally connected and of which we want to be proud. We may also share a sense of freedom in wearing clothing of choice, the ability to experience psychologically satisfying sexual interaction for this first time, being recognised at last as a member of the gender category in which we feel most comfortable, and the sense of doing something for ourselves rather than always trying to please others. (p.207).

Transitioning is an important aspect in the life of a transman and body dysphoria is omnipresent in these autobiographies. Butler's (1999) response to sex reassignment surgery implies that if bodily markers 'indicate' sex, then sex is different to the means through which it is articulated (p.90). To conceive of oneself as either a woman with a penis or a man with a vagina 
is considered pathological as Stone (1991) mentions that, "under the binary phallocratic founding myth by which Western bodies subjects are authorized, only one body per gendered subject is 'right' (p.297). According to the imposed order, one can only be one or the other, not both and certainly not neither, regardless of choice. Yet, the order does not present individuals from challenging, and thus subverting it. Valerio (2006) in the process of exploring his sexuality as a male, describes his experiences with his female partners. For Valerio, he discovered his sexuality during his intimate encounters with female partners. He mentions, "I want to be with a partner who will ignore my female parts. I've come to the realization that I have to be seen as male with a woman in bed in order to be aroused" (p.69). He discovered much later that, "my male identity was deep, rooted in my body. That finally, like all transsexuals, the body is the issue and not the role" (p.101). Body dysphoria is experienced by Valerio too when he finds the incongruity between his gender role and gender identity. The chapter in his autobiography "Ephiphanies" states;

I'm actually a man looking from the eyes of a woman, looking out of the body of a woman, which I see in front of me. The moving backdrop of my life, a feeling of being male-not so much a man in a woman's body as a man with a woman's body. When I was a child, I could feel my boy self-peering out of my eyes, knowing that other people were seeing a little girl (p.106).

This statement implies that the body is a medium through which gendered identities present themselves and it is through body that one communicates to others. It also establishes the importance of body and corporeality in the life of transsexuals as a means to express their identity.

\section{Erasing Femininity}

According to transsexual discourses, "FTMs and transmen are obsessed with ridding themselves of breasts and internal female organs" (Cromwell, p.112) and with "idea of having a penis" (Lothstein, 1983, p.13; Steiner, 1985, p. 353). Money and Brennan in "Sexual Dimorphism in the Psychology of Female Transsexuals" (1968) mentions that, in case of FTMs, "I have never met an FTM or transman who did not want chest 
surgery. Breasts are the primary sign of woman and by implication femininity, to reject them acknowledges the fact" (p.496). Valerio (2006) mentions,

I wear layers. I am lucky since I don't have huge breasts. I only have to wear a couple of pieces of clothing to disguise the bulging contour. A T-shirt and a heavier shirt over it. I can get away with only a t-shirt too since the binder makes me flat, but then I am paranoid about my binding showing (p.218).

Similarly, Khosla (2006) in his autobiography mentions, "I told him about my prior breast reduction and showed him T- shaped scars. I wanted absolutely flat pecs, and I didn't want to be disappointed a second time" (p.46). He has undergone the chest surgery twice to remove the vestiges of femininity. After his second surgery when he was draped in bandages in hospital he asked Selena if his bandages truly looked flat. "She lifted the blanket and said yes, they definitely looked flat" (p.48). Valerio's autobiography (2006) also mentions the anxiety that transmen go through without undergoing the chest surgery.

Taking off breasts will be like unfastening a couple of deflated balloons. I want that contour tight and masculine. I fantasize about finally being able to work out in a gym without worrying about the binding. Being able to see my pecs, instead of having them disappear under my flesh. Being seen as a man, feeling manly in my body without these barriers. I can't wait. I can't wait, but I have to (p.220).

Breasts are considered as important aspect that would change the appearance, as the chest is the body part most likely to block them from living full lives. It inhibits them from living as man and causes discomfort with their identity as a man.

\section{Conclusion}

Transmen are gendered identities with a masculine gender presentation and they undergo surgeries and take hormones to reconstruct their female body as essentially male. As understood from the autobiographical narratives 
taken for the study, transmen prefer masculine pronouns and equally important is 'passing' as 'man' is an important aspect which constructs their identity as male. From Jamison Green (2004) to Max Wolf Valerio's (2006) to Dhillon Khosla's (2006) transition for transmen can be described as a process which includes the transformation of the body and corporeality to bridge the gap and maintain congruity between its visible signifiers of gender i.e., body and the internal invisible 'self'. The (FTM) transsexual autobiographies are taken to explore the question of their representation by locating the assumptions related to masculinity within parameters of performativity and body. Transsexual autobiographies can be considered as an important tool in the non-transsexual's understanding of an unimaginable life, as well as positioning that transsexuals are not alone in their female-bodied experience. Green (2004) in his autobiography asserts that, "Surgery is not what transsexualism is ultimately about" (p.89). The body modifications add on the 'trans' identity of the transsexuals but are not just limited within the corporeality.

\section{References}

Aboim, S. (2016). Trans-masculinites, Embodiments and the Materiality of Gender: Bridging the Gap. NORMA: International Journal of Masculinity Studies, 11.4, 225-236. doi:

10.1080/18902138.2016.1259848

Ames, J. (2005). Sexual Metamorphosis: An Anthology of Transsexual

Memoirs. New York: Vintage Books.

Beauvoir, S. (1949). The Second Sex. New York: Vintage Books.

Beatie, T. (2008). Labor of Love: The Story of One Man's Extraordinary

Pregnancy. CA: Seal Press.

Beasley, C. (2005). Gender and Sexuality: Critical Theories, Critical

Thinkers. London: SAGE Publications.

Bettcher, T.M. (2014). Trapped in the Wrong Theory: Rethinking Trans

Oppression and Resistance. Signs. Journal of Women in Culture and Society, 39(2), 383-406. doi: 10.1086/673088

Beynon, J. (2002). Masculinities and Culture. Buckingham, Philadelphia:

Open University Press. 
Boddy, J. (1995). The Body Nearer the Self. American Anthropologist, 97(1), 134-137. doi: 10.1525/aa.1995.97.1.02a00190

Bolin, A. (1988). In Search of Eve: Transsexual Rites of Passage. South Hadley: Bergin and Garvey.

Bristow, J. (1997). Sexuality. New York: Routledge.

Butler, J. (1999). Gender Trouble. New York: Routledge.

Butler.J. (1993). Bodies that Matter. USA and CA: Routledge.

Califia, P. (1997). Sex Changes: The Politics of Transgenderism. Nova Jersey: Cleis Press Inc.

Connell, R. (2012). Transsexual Women and Feminist Thought: Towards New Understanding and New Politics. Signs, 37(4), 857-881. doi:

$10.1086 / 664478$

Creswell, J.W. (2007). Qualitative Inquiry and Research Design: Choosing Among Five Approaches. Thousand Oaks CA: Sage.

Cromwell, J. (1999). Transmen and FTMs: Identities, Bodies, Genders and Sexuailties. University of Illinois: University of Illinois Press.

Devor, A. (1997). FTM: Female-to-Male Transsexuals in Society.

Bloomington and Indianapolis: Indiana University Press.

Engdahl, U. (2014). Wrong Body. TSQ. Transgender Studies Quarterly, 1(1-2), 267-269. doi: 10.1215/23289252-2400226

Ekins and King. (2006). The Transgender Phenomenon. London: SAGE Publications Inc.

Fairclough, N. (2001). The Discourse of new Labor: Critical Discourse Analysis. In M. Wetherall, S. Taylor \& S.J. Yates (Eds.), Discourse as Data: A Guide for Analysis (pp. 229-266). London: Sage.

Foucault, M. (1977). The History of Sexuality: The Will to Knowledge.

London: Penguin Books.

Friedli, L. (1987). "Passing Women": A study of Gender Boundaries in the Eighteenth Century", In G.S. Rousseau \& R. Porter (Eds.), Sexual Underworlds of the Enlightment (pp. 234-260). Manchester: Manchester University Press.

Goffman, E. (1959). Asylums: Essays on the Social Situation of Mental Patients and Other Inmates. Chicago: Aldine Publishing Company. Green, J. (2004). Becoming a Visible Man. Nashville: Vanderbilt University Press. 
276 Tanupriya \& Pannikot-Performativity in Transsexuals

Green, R. (1987). The Sissy Boy Syndrome and the Development of Homosexuality. New Haven: Yale University Press.

Halberstam, J. (1998). Female Masculinity. Durham: Duke University

Press.

Hausman, B. (2006). Body, Technology, and Gender in Transsexual

Autobiographies. In S. Stryker \& S. Whittle (Eds.), The Transgender

Studies Reader (pp. 335-361). London: Routledge.

Hausman, B. (1999). Virtual Sex, Real Gender: Body and Identity in

Transgender Discourse. In M.A. O'Farrell \& L. Vallone (Eds.)

Virtual Gender: Fantasies of Subjectivity and Embodiment (pp. 190-

216). Ann Arbor: University of Michigan Press.

Herrmann, A. (1991). Passing Women, Performing Men. In L. Goldstein

(Ed.) The Female Body: Figures, Styles, Speculations (pp. 179-189).

Ann Arbor: University of Michigan Press.

Jorgenson, C. (1967). Christine Jorgenson: A Personal Autobiography.

New York: Park S. Ericksson Inc.

Kailey, M. (2005). Just Add Hormones: An Insider's Guide to the

Transsexual Experience. Boston: Beacon Press.

Khosla, D. (2006). Both Sides now: One Man's journey through

Womanhood. San Francisco: Untreed Reads.

Krueger, D.W. (1983). Diagnostic and Management of Gender Dysphoria.

In W. E. Fann, I. Karacan, A.D. Pokorny \& R.L. Williams (Ed.)

Phenomenology and Treatment of Psychosexual Disorders. New

York: SP Medical and Scientific Books.

Lothstien, L. (1983). Female-to-Male Transsexualism: Historical, Clinical, and Theoritical Issues. Boston: Routledge and Kegan Paul.

Martino, M. (1977). Emergence: A Transsexual Autobiography. New York: Crown Publishers.

Meyerowitz, J. (1998). Sex Change and the Popular Press: Historical Notes on Transsexuality in the United States, 1930-1955. GLQ: A Journal of Lesbian and Gay Studies, 4(2), 159-187. doi: 10.1215/106426844-2-159

Money, J., \& Brennan, J.G. (1968). Sexual Dimorphism in the Psychology of Female Transsexuals. Journal of Nervous and Mental Diseases, 147(5), 487-499. doi: 10.1097/00005053-196811000-00005 
Money, J. (1990). Foreword. In S. Nanda, Neither Man nor Woman: The Hijras of India (pp. xi-xiv). Belmont: Wadsworth Publishing Company.

Morris, J. (1974). Conundrum: An Extraordinary Narrative of

Transsexualism. New York: Henry Holt and Company Inc.

Pauly, I. (1974). Female Transsexualism: Part I. Archives of Sexual Behavior, 3(6), 487-507. doi: 10.1007/BF01541134

Paris, R.A. (2005). Transman-Bitesize: The Story of a Woman who became a Man. Bloomington, Indiana: Author House.

Ponse, B. (1978). Identities in the Lesbian World: The Social Construction of Self. Westport: Greenwood Press.

Prosser, J. (2006). Judith Butler: Queer Feminism, Transgender and the Transubstantiation of Sex. In Stryker \& Whittle (Eds.), The Transgender Studies Reader (pp.257-281). New York: Taylor and Francis Group.

Prosser, J. (1998). Mirror Images: Transsexuality and Autobiography. In. Second Skins: The Body Narratives of Transsexuality (pp. 99-134).

New York: Colombia University Press.

Purvis, T. (2006). Sexualities. In P. Waugh (Ed.), Literary Theory and

Criticism: An Oxford Guide (pp. 427-450). New Delhi: Oxford University Press.

Raj, S. (2011). 'Bodies in New Territories': Mapping Masculinity, Gender

Performativity and FTM embodiment in Jamison Green's Becoming a Visible Man. Altitude: an e-Journal of Emerging Humanities Work, 1-19.

Rees, M. (1996). Dear Sir or Madam? The Autobiography of a Female-toMale. London: Cassell Wellington House.

Rubin, H. (2008). Self-Made Men: Identity and Embodiment among

Transsexual Men. Nashville: Vanderbilt University Press.

Steiner, B. (1985). Transsexuals, Transvestities, and Their Partners. In B.

Steiner (Ed.), Gender Dysphoria: Development, Research, Management (pp. 351-64) New York: Plenum Press.

Stoller, R. (1975). Sex and Gender. The Transsexual Experiment. London: Hogarth. 
Stoller, R. (1965). Passing and Continnum of Gender Identity. In J. Marmor (Ed.), Sexual Inversion: The Multiple Roots of Homosexuality (pp. 190-219) New York: Basic Books.

Stone, S. (1991). The Empire Strikes Back: A Posttransexual Manifesto. In J. Epstein \& K. Straub (Eds), Body Guards: The Cultural Politics of Gender Ambiguity (pp. 280-304). New York: Routledge.

Thompson and Sewell. (1995). What Took You So Long? A Girl's Journey to Manhood. UK: Penguin Books Ltd.

Valerio, M.W. (2006). The Testosterone Files. Emeryville: Seal Press. Weigert, A.J., Teitge, J.S., \& Teitge, D.W. (1986). Society and Identity:

Toward a Sociological Psychology. Cambridge: Cambridge University Press.

Whittle, S. (2002). Respect and Equality: Transsexual and Trangender Rights. London: Cavendish Publishing.

Tanupriya, School of Management, National Institute of Technology Karnataka, Dakshina Kannada, Karnataka, India-575025.

Dhishna Pannikot, School of Management, National Institute of Technology Karnataka, Dakshina Kannada, Karnataka, India-575025.

Contact Address: Direct correspondence to Dr. Dhishna Pannikot, email: dr.dhishnapannikot@gmail.com 


\section{Hipatia Press}

Instructions for authors, subscriptions and further details:

http://mcs.hipatiapress.com

\section{Bidirectional Violence among Male and Female University Students: Comparison of Observations and Results between Two Countries}

Teresa Fernández de Juan ${ }^{1}$ \& Lourdes Florez Madan²

1) El Colegio de la Frontera Norte, México

2) Universidad de la Tercera Edad, Dominican Republic

Date of publication: October $21^{\text {st }}, 2018$

Edition period: February 2019 - June 2019

To cite this article: Fernández de Juan, T \& Florez Madan, L. (2018). Bidirectional Violence among Male and Female University Students: Comparison of Observations and Results between Two Countries. Masculinities and Social Change,7(3),279-312. doi: 10.17583/MCS.2018.3499

To link this article: http://doi.org/10.17583/MCS.2018.3499

\section{PLEASE SCROLL DOWN FOR ARTICLE}

The terms and conditions of use are related to the Open Journal System and to Creative Commons Attribution License (CC-BY). 
MCS - Masculinities and Social Change Vol. 7 No. 3 October 2018

pp. 279-312

\section{Bidirectional Violence among Male and Female University Students:}

\section{Comparison of Observations and}

Results between Two Countries

Teresa Fernández de Juan

El Colegio de la Frontera Norte
Lourdes Florez Madan

Universidad de la Tercera Edad

\section{Abstract}

This paper presents the results of a research project that included the application of a survey in 597 university students in Santo Domingo, Dominican Republic, and Tijuana, México, whose central aim was to examine the degree of violence in intimate couples, including that of men towards women and women against men, in different countries. It also explores the level of knowledge and analyzes the degree of awareness that the samples have about existing abuse. The results show similarity in the exercise of violence by both sexes; aspects of hegemonic masculinity that still permeates both, and the need for studies that have a real impact on education against this problem. This, irrespectively of age and socio-economic stratum to which both sexes belong.

Keywords: Perception of violence, bidirectional violence, heterosexual couples, Tijuana, Santo Domingo. 
pp. 279-310

\section{Violencia bidireccional entre hombres y mujeres universitarias: Resultados y observaciones al comparar entre dos países}

Teresa Fernández de Juan

El Colegio de la Frontera Norte
Lourdes Florez Madan

Universidad de la Tercera Edad

\section{Resumen}

Este trabajo expone los resultados de una investigación que incluyó la aplicación de una encuesta a 597 universitarios de Santo Domingo, República Dominicana y Tijuana, México, y cuyo objetivo central fue examinar el grado de violencia en la pareja tanto de hombres hacia mujeres como de mujeres hacia hombres, en diferentes países. También explora el nivel de conocimiento y analiza el grado de concientización que las muestras poseen acerca del maltrato existente. Los resultados demuestran la similitud en el ejercicio de la violencia por parte de ambos sexos; aspectos de masculinidad hegemónica que aún permean a ambos, y la necesidad de implementar estudios que ejerzan un impacto real en la educación contra esta problemática. Esto, con independencia de la edad y el estrato socioeconómico al cual ambos sexos pertenezcan.

Palabras clave: Percepción de la violencia, violencia bidireccional, parejas heterosexuales, Tijuana, Santo Domingo. 


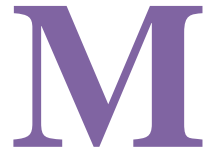

ost studies about intimate partner violence are limited to addressing men's abusive behavior towards women by highlighting the patterns of masculinity and femininity established within the framework of patriarchal culture.

However, over the years research has shown that this abuse is bidirectional, therefore demonstrating that both sexes equally play the roles of perpetrator and victim, especially among young couples (Shook, Gerrity, Jurich, \& Segrist, 2000; Muñoz, Graña, O’Leary, \& Gonzalez, 2007; Argoff, 2009; Goinheix’s, 2012; Martínez, Vargas, \& Novoa, 2016; Aparecida, da Cruz, Coelho, Fiqueira, \& Carvalho, 2016).

Several articles confirm this to be so in the case for Mexico (see Cáceres, 2007; Hernández, 2007; Trujano, Martínez, \& Camacho, 2010; Alegría \& Rodríguez, 2015). In 2012, the Instituto Mexicano de la Juventud (Mexican Institute for Youth) announced that about 30 percent of Mexican men and women between the ages of 12 and 29 had been victims of violent behavior in their intimate relationships (Méndez, 2012).

The Encuesta de Salud Reproductiva en la Adolescencia de Baja California (Survey of Reproductive Health in Adolescents of Baja California) found that 47.6 percent of the youth population in Baja California experienced some type of non-extreme violence from their intimate partner. This result was only six percentage points below the number of young women who found themselves in the same situation (El Colef, 2006). The records indicate that the predominant type of violence was psychological, with more than 70 percent directed against males (González \& Fernández, 2014). This concurs with what has been found by other authors in different parts of Mexico, such as Moral and López (2012).

Delving further into this matter, Ramírez (2002) pointed out the difficulty - and even the fear - felt by many men for not fulfilling their socially assigned role as the dominant partner. This implies that it is not enough to identify these abusive behaviors, but that it is also necessary to provide specific coping tools and skills. On the other hand, it also indicates that in certain contexts men are being educated in a non-traditional manner in which they are taught to respect women "which may explain why many of them, although they perceive their female partners' behavior as violent, learn to tolerate and live with it" (Ramírez, 2002, p. 350). 
This situation surpasses not only gender differences but also differences in educational and socio-economic status. Furthermore, violence is frequently reported among college-age students regardless of their academic field of study and country of origin (Soriano, 2011; Olvera, Arias, \& Amador, 2012; UCM, 2013; Moral \& López, 2013; Fernández, 2014; Mohamed, Herrera, \& Carracedo, 2014; Formental, Hernández, \& Fernández, 2014; Rodríguez, 2015; Sosa \& Menkes, 2016; Aparecida et al. 2016; Cubillas, Valdez, Domínguez, Román, Hernández \& Zapata, 2016).

For these reasons, this study was designed to investigate the magnitude of violence between both sexes among college student couples of medium to high socioeconomic status, both in the city of Tijuana, Mexico and in Santo Domingo, Dominican Republic. Very few studies of this nature have been carried out in Tijuana, and it is the first study in Santo Domingo that explores bidirectional violence, including violence perpetrated by women towards men.

This binational research paper ${ }^{1}$ presents concrete findings aiming to delve deeper into the issue of violence between both sexes that live as couples, addresses their degree of knowledge and awareness about violence and explores the influence of hegemonic masculinity that remains in these two different cultures.

\section{Methodology}

The survey for this study was designed and validated for both Tijuana, Mexico (Fernández, Martínez, Unzueta, \& Rojas, 2016) and Santo Domingo, Dominican Republic (Rosales, Florez, \& Fernández, 2017). While all survey participants were currently enrolled in a university, there was a wide range of age variation (between the ages of 18 to 63). The age variation did not reveal enough dissimilarity to be considered as a differentiating or determining variable. It should be noted that participation in the survey was anonymous, voluntary and strictly confidential ${ }^{2}$. Participants were university students currently in a relationship with the same partner during at least the past year.

The fundamental variables considered were: 
a) Sex (male or female)
b) Type of violence (economic, sexual, psychological and physical). For this variable we considered de definitions already established by other authors (Moreno, 1999; Amor, Echeburúa, De Corral, Zubizarreta \& Zarazúa, 2002, Ruiz, Plazaola \& Del Río, 2007; García, Pico, Sánchez, Savall, Celda, Blasco \& Martínez, 2005; Fernández \& Pérez, 2007; Pico, Echeburúa \& Martínez, 2008) ${ }^{3}$

c) And level of awareness of violence (by victim and/or victimizer ${ }^{4}$ ). This was achieved by contrasting the first part of the questionnaire, consisting of 14 open questions that assess their general degree of awareness about the presence of violence and ability to discern between different types of violent behavior. This part of the questionnaire poses questions such as: What does intimate partner violence mean to you? Do you think you have ever exerted, or been a victim of violence? It also measures whether they are capable of identifying when they have been a victim or perpetrator of violence (see Fernández et al. 2016; and Rosales et al. 2017). The last part of the questionnaire emphasizes specific aspects of each type of violence by introducing such questions as, for example: Has your intimate partner ever yelled at you in public? Have you ever yelled at your intimate partner in public? It also asks about various manipulative behaviors associated with sexual, psychological or economic abuse, some of which might have previously been perceived as normal. The entire questionnaire was designed and tested to gradually raise the subjects' awareness about behaviors which are in fact manifestations of violence.

The data was processed using SPSS Statistics v19 in order to determine the presence or absence of intimate partner violence among university students. Comparisons by sex and by type of violence were analyzed and evaluated according to Pearson's chi-square statistical test $(\chi 2)$ (bilateral significance). Each of the items was encoded as a dichotomous variable. Once the data was captured, its frequencies were analyzed and each response was differentiated based on sex. Nonparametric tests were used to analyze the correspondence between sex and each of the violence types and conditions (victim or victimizer), using the $\chi^{2}$ test with a level of significance of 0.05 after the Kolmogorov-Smirnov test to verify the relationship between the variables of interest. 


\section{Study sample}

The study sample was random, non-probabilistic and by convenience. 5 The selection criteria was to include students from private schools with a medium-high socioeconomic level, which comprised a total of 597 university students of medium to high socioeconomic status of which 181 were male, and 416 were female. In the case of the City of Tijuana, the group consisted of 366 people, 123 men, and 243 women. Their ages ranged between 18 and 63 years. The greatest percentage of participants was in the age group of 20 through 25 years, and the next largest group consisted of students between 18 to 19 years of age. The students attended the Universidad de la Tercera Edad (University of the Elderly: UTE) in Santo Domingo in the Dominican Republic, where the group totaled 231 students, of which 173 were female and 58 were male. Ages fluctuated between 20 and 63 years of age, a majority of which ranged between 31 and 50 years old. Table 1 shows the total study sample's distribution.

Table 1

Composition of the study sample and sub-samples

\begin{tabular}{cccc}
\hline & \multicolumn{3}{c}{ City } \\
\cline { 2 - 4 } Sex & Tijuana & Santo Domingo & Sample total \\
\hline Male & 123 & 58 & 181 \\
Female & 243 & 173 & 416 \\
Total & 366 & 231 & 597 \\
\hline
\end{tabular}

Source. Prepared by the authors

\section{Results}

Table 2 shows that less than half of the university students surveyed in Santo Domingo and Tijuana recognized having experienced (currently or in the immediate past) intimate partner violence while in a couple relationship at the start of the survey. That shows that the majority of participants from both countries (and particularly the Tijuana men) weren't aware or had knowledge about being in a violent intimate relationship, be as victims and/or abusers. 
Table 2

Initial recognition of intimate partner violence (shown by their responses to the first 14 questions) in both Santo Domingo and Tijuana

\begin{tabular}{llccccc}
\hline City & \multicolumn{3}{c}{ Sex } & \multicolumn{2}{c}{ Total } \\
\hline Santo Domingo & \multicolumn{2}{c}{ Female (173) } & \multicolumn{2}{c}{ Male (58) } & \multicolumn{2}{c}{ Total (231) } \\
\cline { 2 - 7 } & ct. & $\%$ & ct. & $\%$ & ct. & $\%$ \\
\cline { 2 - 7 } & 76 & 43.9 & 28 & 48.3 & 104 & 45 \\
\multirow{2}{*}{ Tijuana } & \multicolumn{2}{c}{ Female (241) } & Male (125) & \multicolumn{2}{c}{ Total (366) } \\
\cline { 2 - 7 } & ct. & $\%$ & ct. & $\%$ & ct. & $\%$ \\
\cline { 2 - 7 } & 97 & 72.4 & 37 & 27.6 & 134 & 36.6 \\
\hline
\end{tabular}

Source. Prepared by the authors. Note. ct. $=$ count

The largest number of participants who recognized having experienced violence in their relationships (suffered or exercised) was found among the Tijuana women. This could be influenced by the fact that this Mexican city has a greater number of working women compared to other cities in the country. Most of them are economically independent, and there are a high number of women who are heads of households, which can be related to a greater initial violence awareness and knowledge about their autonomy possibilities. This feminine economic emancipation scenario does not occur in Santo Domingo, where it should be noted, as show in Table 2, that even though there were no significant differences between sexes (and the number of participants who perceived themselves as being victims of violence was less than half of entire study sample), in Tijuana there are more men who point out the existence of intimate partner violence. 
Table 3

Comparison of responses to the last part of the questionnaire about intimate partner violence in both Santo Domingo and Tijuana

\begin{tabular}{|c|c|c|c|c|c|c|}
\hline \multirow{2}{*}{$\begin{array}{l}\text { City } \\
\text { Santo Domingo }\end{array}$} & \multicolumn{4}{|c|}{ Sex } & \multirow{2}{*}{\multicolumn{2}{|c|}{$\begin{array}{c}\text { Total } \\
\text { Total }(231)\end{array}$}} \\
\hline & \multicolumn{2}{|c|}{ Female (173) } & \multicolumn{2}{|c|}{ Male (58) } & & \\
\hline & ct. & $\%$ & ct. & $\%$ & ct. & $\%$ \\
\hline & 129 & 74.5 & 48 & 82.7 & 177 & 76.6 \\
\hline \multirow[t]{3}{*}{ Tijuana } & \multicolumn{2}{|c|}{ Female (241) } & \multicolumn{2}{|c|}{ Male (125) } & \multicolumn{2}{|c|}{ Total (366) } \\
\hline & ct. & $\%$ & ct. & $\%$ & ct. & $\%$ \\
\hline & 188 & 78 & 98 & 78.4 & 286 & 78.1 \\
\hline
\end{tabular}

Source. Prepared by the authors. Note. ct. $=$ count

When comparing the answers shown in the first (Table 2) and last (Table 3) parts of the questionnaire, the resulting differences display the degree of unawareness in each sample with respect to identifying when violence was occurring, and what types of violence were taking place in the couple's relationship. For example, in the case of Tijuana, out of a total of 245 victimizers, 136 were unaware that they were abusing their partner because they did not regard abusive behaviors as violent. The fact that the violent actions were considered normal could partially explain that they would go unnoticed. The percentage of women who recognized that they were being victimized or acting as victimizers in both Santo Domingo and Tijuana is very similar, after having completed the last part of the questionnaire and did not show differences by sex in either case. To delve further into this matter, Table 4 shows the following comparison. 


\section{MCS - Masculinities and Social Change, 7(3) 287}

Table 4

Comparison between responses to first and last parts of the questionnaire as indicators of the respondents' awareness of having experienced intimate partner violence (IPV) in Tijuana and Santo Domingo

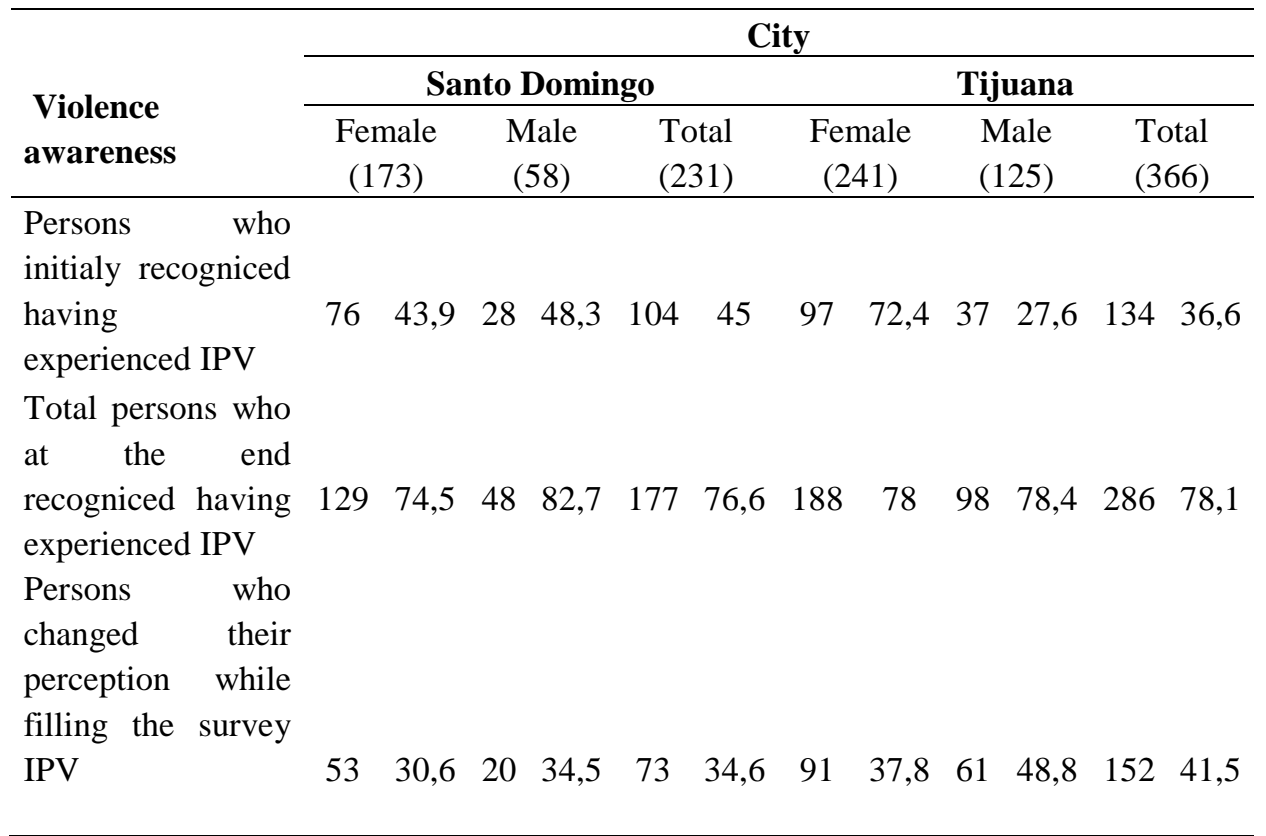

Source. Prepared by the authors. Notes. ct. = count; IPV = Intimate Partner Violence.

As previously discussed, this questionnaire addresses general awareness only up to question 14, after which it examines the specific aspects characterizing the four types of violence studied, as a means of raising participants' awareness about the presence of violence in its different forms and degrees in both roles as victim and victimizer. Table 4 shows how the number of cases who recognize the existence of violence in their intimate relationship, jump from less than half (46 percent in Santo Domingo and 36.6 percent in Tijuana) to a majority when we comparing the beginning and end of the survey. In regard to sex, it's worth noting, in the case of Tijuana, the high difference shown between women (72.4 percent) and men 
(27.6 percent) about recognizing violence in their relationship at the beginning of the survey, situation that is latter recognized by both sexes at the end of the survey.

These findings highlight the degree of respondents' unawareness, regardless of sex, age and country, in so far as their ability to distinguish between different types of violence within their couple relationship, taking into account the fact that in many cases, respondents' knowledge may have been distorted prior to the survey.

It is important to add that in our study there were no age-related differences even when the average age ratios in the samples from both countries were unequal. Furthermore, these results concur with those found by Soriano (2011) and other authors like Moral and Lopez (2013), whose samples ranged from 18 to 63 years of age.

Table 5

Types of intimate partner violence in respondents in Tijuana and Santo Domingo

\section{City}

\begin{tabular}{lcccccccccccc}
\cline { 2 - 12 } Type of violence & \multicolumn{4}{c}{ Tijuana } & \multicolumn{4}{c}{ Santo Domingo } \\
\cline { 2 - 13 } & $\begin{array}{c}\text { Female } \\
(241)\end{array}$ & \multicolumn{1}{c}{ Male } & \multicolumn{1}{c}{ Total } & \multicolumn{2}{c}{ Female } & \multicolumn{2}{c}{ Male } & \multicolumn{2}{c}{ Total } \\
& ct. & $\%$ & ct. & $\%$ & ct. & $\%$ & ct. & $\%$ & ct. & $\%$ & ct. & $\%$ \\
\hline Psicological & 186 & 77,2 & 93 & 74,4 & 279 & 76,2 & 164 & 94,8 & 55 & 94,8 & 219 & 94,8 \\
Physical & 68 & 28,2 & 41 & 32,8 & 109 & 29,8 & 151 & 87,3 & 48 & 82,8 & 199 & 86,1 \\
Sexual & 27 & 11,2 & 18 & 14,4 & 45 & 18,3 & 9 & 5,2 & 5 & 8,6 & 14 & 6,1 \\
Economical & 39 & 16,2 & 28 & 22,4 & 67 & 12,3 & 8 & 4,6 & 5 & 8,6 & 13 & 5,6 \\
\hline
\end{tabular}

Source. Prepared by the authors. Note. ct. $=$ count

Table 5 shows that the most prevalent type of violence upon completion of the last part of the questionnaire was psychological abuse, which was experienced by 94.8 percent of couples in Santo Domingo and 76.2 percent in Tijuana, regardless of gender in both countries. Nevertheless, when comparing violence of the physical type, which is the one that stands in second place of occurrence in both countries and also without sex 
differences, it shows a high incidence for the case of Santo Domingo (with 86.1 percent vs 29.8 percent in Tijuana), also without significant differences between men and women.

Men reported experiencing psychological violence and felt undervalued by their female partners in instances when they could not afford to invite their women out on a date or when economic hardship prevented them from assuming the traditional role of financial provider in their relationships, associated with learned and expected patterns of masculinity. It was surprising for the authors to find that economic violence did not significantly stand out in the Santo Domingo sample.

This study reveals the high number of both men and women who suffer intimate partner violence without knowing it.

In this regard, we believe it's relevant to add some results found in previous studies made with this project, related to being victims or victimizers by sex: In the Santo Domingo Study Sample (Rosales \& Florez, 2016), even though many women did not recognize being abusers at the beginning of the survey, at the end this changed, as it's shown in table 6 .

Table 6

Victims and victimizers: comparative evaluation by sex and type of violence in Santo Domingo

\begin{tabular}{lcccc}
\hline \multirow{2}{*}{ Type of violence } & \multicolumn{2}{c}{ Victim } & \multicolumn{2}{c}{ Victimizer } \\
\cline { 2 - 5 } & Female & Male & Female & Male \\
\hline Physical & $28,9 \%$ & $29,3 \%$ & $24,9 \%$ & $19,0 \%$ \\
Psychological & $55,5 \%$ & $62,1 \%$ & $56,6 \%$ & $60,3 \%$ \\
Sexual & $24,3 \%$ & $25,9 \%$ & $31,8 \%$ & $18,9 \%$ \\
Economic & $28,9 \%$ & $41,4 \%$ & $16,8 \%$ & $15,5 \%$ \\
\hline
\end{tabular}

Source. Own translation based on Rosales \& Florez (2016, p. 44)

In Table 6 we can see that there were no significant differences between man and women in Santo Domingo regarding being victim or victimizer. And, in both cases, psychological violence predominated.

On the other hand, in a previous study carried out for this purpose in Tijuana (Fernández et al., 2016) it was highlighted that almost 80 percent of 
the sample had suffered or exerted violence in their relationship, with no difference between both sexes, being the tendency that men were the most abused and least aggressors, as well as those who were both victims and victimizers, as shown in Table 7.

Table 7

Relationship between being victim or victimizer by sex in Tijuana

\begin{tabular}{lcc}
\hline & Male & Female \\
\cline { 2 - 3 } Victims & $72,8 \%$ & $64,3 \%$ \\
Both victim and victimizer & $60,0 \%$ & $53,9 \%$ \\
Victimizer & $65,6 \%$ & $67,6 \%$ \\
\hline
\end{tabular}

Source. Own translation based on Fernández, Martínez, Unzueta \& Rojas (2016, p. 258).

At the end of the survey it was also evident, as expressed by Fernández \& González (2018), the high number of those who recognized to be suffering or to exert violence (victims or victimizers) in their relationship in both countries, without sex differences. To illustrate this we reproduce a graph elaborated for that study:

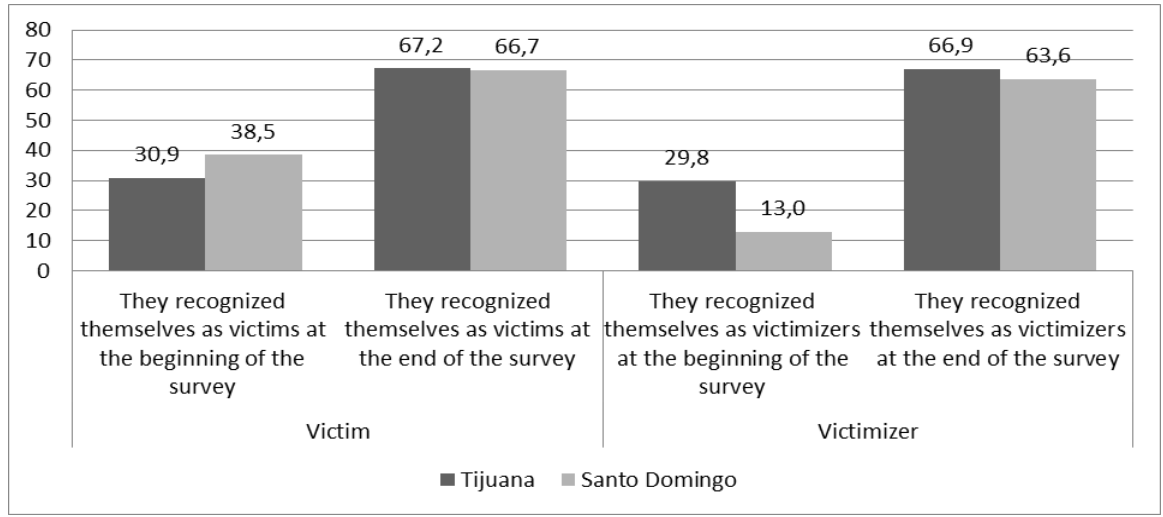

Graph 1. Comparison of victims and victimizers in Tijuana and Santo Domingo, before and after the survey (percentages)

Source. Own translation. Based on Fernández \& González (2018, p. 324) 
It is important to consider the role that guilt plays in the lack of visibility of violence. Escudero, Polo, López and Aguilar (2005), Scott and Straus (2007) and Abelino and Monroy (2016), among others, pointed out that when a person is in the midst of a violent dynamic, the belief tends to be that he or she alone is to blame for the other's reaction and feels guilty as a result. Although it has been observed that men often use this as a tool to exert power, this is not unique to the male sex. In our study, Tables 8 and 9 show the principal causes referenced in both countries.

Table 8

Principal causes identified by respondents as the origin of intimate partner violence in Santo Domingo

\begin{tabular}{|c|c|c|c|c|c|c|}
\hline \multirow[b]{2}{*}{ Cause } & \multicolumn{2}{|c|}{ Female (173) } & \multicolumn{2}{|c|}{ Male (58) } & \multicolumn{2}{|c|}{ Total (231) } \\
\hline & ct. & $\%$ & ct. & $\%$ & ct. & $\%$ \\
\hline Education/culture/machismo/feminism & 85 & 49,1 & 16 & 27,6 & 101 & 43,7 \\
\hline Jealousy/insecurity & 58 & 33,5 & 17 & 29,3 & 75 & 32,5 \\
\hline Poor communication & 54 & 31,2 & 12 & 20,7 & 66 & 28,6 \\
\hline Financial problems & 41 & 23,7 & 12 & 20,7 & 53 & 22,9 \\
\hline
\end{tabular}

Source. Prepared by the authors. Note. ct. $=$ count

In Santo Domingo education and culture (with its underlying machismo) are seen as the most common causes of intimate partner violence along with jealousy and insecurity, poor communication skills and financial hardship. Among the women, the perceived causal factors for violence appeared in the following order:

- Nearly half, (49.1 percent), attributed violence to the patriarchal culture and education imbued with the macho prototype;

- 33.5 percent identified jealousy and insecurity on behalf of their intimate partner as the cause;

- 31.5 percent blamed poor communication;

- 23.7 percent felt that financial hardship was responsible. 
These results are consistent with the study conducted by the Fondo de Población de las Naciones Unidas (United Nations Population Fund) (UNFPA, 2017) in Santo Domingo about women, according to which:

- 60 percent felt that their partner attempted to control them;

- 40 percent complained that their male partner exploded into jealous fits when the woman spoke to other men;

- 34 percent said that what originated the violence was their partner's insistence in knowing where they were at all times;

- 27 percent said that their male partner did not allow them to meet with girlfriends;

- 27 percent attributed violence to their partner's mistrust of their management of money.

In contrast, 29.3 percent of male interviewees indicated insecurity and jealousy as a cause of intimate partner violence, while 20.7 percent blamed poor communication and financial hardship.

In a study on violence in married couples in the Dominican Republic, Bogaert (2014) concluded that there is a direct correlation between child abuse and violence in married couples. The study suggests that a male subject who was exposed to domestic violence during his childhood has a higher propensity to becoming abusive toward his spouse as an adult. This is a repetitive finding in research carried out in other countries, which suggests that in order to discover the motives for intimate partner violence in Santo Domingo it is necessary to interpret the nature of domestic violence in the context of the matrifocal family dynamic. 
Table 9

Principal causes identified by respondents as the origin of intimate partner violence in Tijuana

\begin{tabular}{|c|c|c|c|c|c|c|}
\hline \multirow[b]{2}{*}{ Cause } & \multicolumn{2}{|c|}{ Female (241) } & \multicolumn{2}{|c|}{ Male (125) } & \multicolumn{2}{|c|}{ Total (366) } \\
\hline & ct. & $\%$ & ct. & $\%$ & ct. & $\%$ \\
\hline Jealousy/insecurity & 92 & 38 & 53 & 42 & 145 & 40 \\
\hline Poor communication & 41 & 17 & 31 & 25 & 72 & 20 \\
\hline Education/culture/machismo/feminism & 49 & 20 & 22 & 18 & 71 & 19,4 \\
\hline Financial problems & 23 & 10 & 9 & 7 & 32 & 9 \\
\hline
\end{tabular}

Source. Prepared by the authors. Note. $\mathrm{ct} .=$ count

The sum total of responses in the Tijuana sample show the following root causes of violence: jealousy and insecurity account for 40 percent of the causes identified by respondents; poor communication for 20 percent, followed by 19.4 percent for reasons of education and culture.

Thirty-eight percent of the women identified jealousy and insecurity as the principal causes of violence while 20 percent blamed education and culture. However, 42 to 45 percent of the men named jealousy, insecurity and communication respectively, as the cause of abusive behavior. We are reminded of the research study conducted by Chávez and Rodríguez (2015) in Mexican universities which found that when asked whether "demanding explanations is considered violence", there was a major difference in men's and women's answers. Thirty-five percent of men strongly noted that "demanding explanations is not considered violence", while 70 percent of women replied that "the behavior was in fact, a violent act." Ramírez and Núñez's case study (2010, p. 282) of Mexican university students suggests that: "Developing studies with a multifactorial focus that take into consideration couples' perception about violence may help to reduce risk factors for intimate partner violence".

In contrast with our expectations of finding a higher predominance of ideas associated with the hegemonic masculinity perspective, the results of this study show few differences between both sexes. The latter concurs with Glass, Fredland, Campbell, Yonas, Sharps, and Kub (2003), who states that the abuser-abused pattern cannot be assumed, given the equal distribution 
of power. This study shows that women not only consider themselves equal but they tend to surpass men in some types of abuse.

\section{Discussion}

This study demonstrates that there exists, not only a high degree of violence between couples in universities in Santo Domingo and Tijuana, but that violence occurs without showing significant discrepancies between both sexes associated with psychological and physical abuse.

Although some research has been carried out in Tijuana (Fernández, 2014; González \& Fernández, 2014; Fernández et al. 2016) about this subject, in the case of the Dominican Republic this approach of the matter is completely new ${ }^{7}$, and no previous studies have taken place to explore violence of women towards men as far as we know.

The findings of high rates of abuse in both sexes are consistent with other researchers', such as those documented by Swahn, Alemdar, and Whitaker (2010). These studies have pointed out that it is appropriate to think about these intimate couples as implicated in aggressive behaviors rather than as static victim-aggressor roles (Nocentini, Menesini, \& Pastorelli, 2010; Adam, 2013; Zamudio, 2014).

A very low percentage of the students, even in the context of higher education, tend to be unaware of having experienced intimate partner violence regardless of sex, whereby they often do not consider themselves either victims or abusers in their couple relationships.

Soriano highlights the frequency with which abuse and offenses are misidentified as love and interest on behalf of romantic partners:

We believe that this is one of the reasons why they [...] do not break off their relationship. This normalization of violence in patterns of daily interactions is one of the factors that cause violence to seep into relationships, are consented to, and thus become perpetuated. (Soriano, 2011, p. 96)

These findings are consistent with those found by Zamudio, Ayala and Andrade (2011, p.4) in their study on students from the Universidad Autónoma de Chapingo (Autonomous University of Chapingo, in Mexico). 
According to data obtained by a survey conducted by these authors, "when asked if while at Chapingo they had ever been attacked, only 36 percent of the men and 28 percent of the women answered affirmatively." However, after walking them through the process of identifying the different types of violence, both sexes confirmed having been victims of violence. This study also found that both men and women experience violence in similar percentages ( 84 percent of men vs. 83 percent of women). In a study carried out in 2005 with 500 students from the Universidad de Colombia - Bogotá (National University of Colombia in Bogotá) Amórtegui-Osorio (2015) also found that couples involved in violent acts (without distinction between sexes) tended to believe that in many instances violence was acceptable and justified.

The type of violence most detected at the end of the survey was psychological with a high percentage in both countries, followed by the physical type (mostly in the case of Santo Domingo), also without difference between men and women.

Other studies with Mexican couples (Rojas-Solis, 2013) also found psychological violence to be the most prevalent (Ramírez \& Smithey, 2008) in coexistence with other types of abuse (Olvera et al. 2012) experienced almost equally by men and women (Rangel \& García, 2010).

Soriano (2011) found that amongst the group of women who acknowledged being victims of violent behavior, all reported having been subjected to different subcategories of psychological abuse. A significantly higher incidence (32.1 percent) of these cases involved situations associated with controlling behaviors. Studies by Abelino and Monroy (2016) found that most of the young women who experienced psychological violence by their intimate partners attributed it to their partners' lack of appreciation for their professional careers. They reported feeling undervalued by their male partners who held their own professional goals in higher regard than theirs. This was consistent with their internalized beliefs associated with the patriarchal model.

Men reported experiencing psychological violence and felt undervalued by their female partners in instances when they could not afford to invite their women out on a date or when economic hardship prevented them from assuming the traditional role of financial provider in their relationships, associated with learned and expected patterns of masculinity. As has been 
ratified by the Dominican psychologist Soraya Lara, President of the Patronato de Ayuda a Casos de Mujeres Maltratadas (Board of Trustees in Support of Battered Women) (cited by Mejía, 2011), Dominican women usually see men mostly as providers. Consequently, when men fail to meet this expectation, they are subjected to more pressure than was observed within this sample. It is often for this reason that women tolerate violence from their male partner and do not report incidents to specialized centers, where statistics would otherwise be recorded on the subject, and made available for research.

Chávez and Rodríguez (2015) also found evidence of this perception in their samples but showed that a higher number of men (30 percent) rather than women (10 percent) were convinced that being the primary financial provider is an essential part of being a man. We concur with Rodriguez (2014) that this worldview, encompassing the traditional patriarchal model that upholds the belief that men should support women financially (and that women should take care of men in return for being supported by them), is entrenched in both men and women. He quotes Molina (cited by Tubert, 2003, p. 143), who emphasizes that a key and multifaceted feature that enables the perpetuation of the patriarchal model is "the recognition, and to a certain degree, the complicity of women." The latter has proven to influence mutual appreciation and greater self-esteem on behalf of both sexes (Fernández, Pérez, \& Anguiano, 2000). This also reinforces the female's deeply ingrained belief regarding "women as the perennial caregivers of men" (even when the male fails to succeed as the primary household provider) which is also present in other cultures (Formental et al. 2014). Chávez and Rodríguez (2015) found that more than half of the interviewees regardless of sex, held the belief that "women were born with a maternal instinct". This "motherhood mystique," referred to by Rodriguez (2014, p. 175) becomes the safeguard of the accepted patriarchal traditions supporting and validating the oppression to which women are subjected. Furthermore, 20 percent of the sample (Chávez \& Rodríguez, 2015) stated their agreement that "being a man means being aggressive."

Regarding the students' perception of their personal experience, Soriano (2011) found that only 2.6 percent of female students reported having been mistreated or abused by their intimate partner. This data indicates that a significant amount of women are subjected to abuse without being aware of 
it, which is reflected in 97.4 percent of the sample in Soriano's case study. Only 1.2 percent of the men consider they are being abused by their intimate partner, while the other 98.8 percent, according to Soriano, are being subjected to abuse without recognizing it as such. These findings reveal the large number of both men and women who are unknowing victims of intimate partner violence, which is consistent with the findings in this binational study.

Fonseca and Quintero (2008) refer to the lack of visibility of violence against women as a product of their learned tolerance towards abuse, which serves to promote its acceptance and their submissiveness. Furthermore, Chávez and Rodríguez (2015) research developed at the Universidad Nacional Autónoma de México (National Autonomous University of Mexico) and at the Universidad Autónoma de México - Xochimilco (Autonomous University of Mexico - Xochimilco) both located in Mexico City, found that more than half of both sexes experienced violence in their daily lives.

Our astonishment is shared with Montesinos and Carrillo (2011) of Universidad Autónoma de México-Azcapotzalco (Autonomous University of Mexico-Azcapotzalco) who found the coexistence of high levels of violence in all of its forms, along with low visibility of violence in a university setting where its prevalence would be least expected, also belonging to a middle-upper socioeconomic stratum. In this aspect our findings coincide with the research of Dr. Luis Verge, Director of the Centro de Intervención Conductual para Hombres de Santo Domingo (Center for Behavioral Intervention for Men in Santo Domingo), that in an interview with Maria Isabel Soldevila $(2011)^{5}$ pointed out that only 4 percent of men cared-for at this facility were illiterate; 84 percent of violent men were employed; 94 percent did not use drugs, and only three percent had a psychiatric disorder. The latter emphasizes the fact that there is a distorted and stereotyped idea (similar to the results in our study) that men who exert violence have low economic status, are uneducated, use drugs, and are unemployed or have mental disorders.

We believe there is an urgent need to work on developing preventive strategies to help change the perception of what being a man and a woman entails and what is expected of each of their gender roles outside of, and within their intimate partner relationships. 
In agreement with Vélez (2011) and other authors who have pointed out that women have fixed ideas about masculinity, as much as men have preconceived expectations about women's behavior. The need for a change in mindset in members of both sexes is critical, given that, as Cucco (2010, p. 15) states: "socio-political transformations do not move forward if they are not executed along with the liberation of the individual psyche." In our opinion, this requires a deep sexual re-education, beginning with an attitude change on behalf of the trainers themselves. Therefore, the authors of this study agree with Trujano, Martínez and Camacho (2009, p. 352):

Precisely because relationships are socially and historically determined, it is essential to emphasize that a gender perspective implies that relationships that are harboring inequality, domination, discrimination and violence between men and women, can and should change. Hence, the importance of embracing a multiplicity of ways to be masculine as we have recognized the right to a plurality of ways to be feminine. Building new bridges between our differences will open possibilities for complementing the roles of men and women and searching for alternatives and relationships with a sense of equality between men and women.

Few differences were noted from a cultural perspective, despite the fact that the study compared data from two different cultures with distinctive cultural variables that constitute a fundamental ingredient in identity development (Rocha, 2009). Both samples showed resemblances, especially regarding the alarming degree of unawareness of the presence of violence. Even when violence was recognized as such, the results of the survey indicate no significant differences across sexes with regard to psychological abuse, which was most prevalent, or in physical violence, which was less frequent.

We advise being mindful of Folguera's work (2014, cited by Rojas, 2016) which explores the development of masculine identity within the context of significant and rapid socio-cultural transformations that have brought about dissent against traditional imposed values, further complicating men's perspective. When men attempt to live within these new parameters of masculinity without a clear narrative to adhere to, they 
become vulnerable to victimization within their couple relationships in a manner that was previously either non-existent or less visible.

\section{Conclusion}

These results, put to light not only the high level of violence between couples in both countries, even when it relates to a sample composed of students of higher educational level (and this aspect does not appear to be crucial to palliate this problem), in addition it was evident the lack of awareness about gender violence between men and women.

Despite the fact that other studies have observed outstanding awareness gaps about violent behavior associated with the hegemonic masculinity worldview, this study observed a similarity in patterns of violent behavior across sexes, similar to that found by McDonell, Ott \& Mitchell (2010), Kaukinen, Gover y Hartman (2012) y Karakurt and Cumbie (2012), even if it studied different countries and age groups. These findings suggest an alarming trend toward normalization worldwide, across cultural and gender differences.

We agree with Castro-Borunda, Naranja-Cantabrana and AyalaBobadilla (2015) that this evidence indicates a need for permanent sex education programs designed from a gender-specific perspective, in order to help male and female students identify and differentiate between the various types of violence. Given the rise of violence in young couples, this kind of program would serve as a preventive measure. We recommend an interactive and experiential approach to instruction, directly involving facilitators and participants alike.

As we have seen, prior to the completion of the last part of the questionnaire, the male and female respondents in both countries who admitted to having personally experienced intimate partner violence were less than half of the sample. There were relatively slight differences by sex, whereby more men than women in Santo Domingo and more women than men in Tijuana, felt abused. However, when comparing their responses in the first and last parts of the questionnaire, the majority of respondents (almost 77 percent) of both sexes in the two countries admitted to experiencing intimate partner violence. This coincides with findings by other authors from different parts of the world who have been mentioned in this article. 
This study clearly shows that there is a high degree of unawareness, lack of visibility, normalization and ignorance with regard to the occurrence of partner violence and types of abuse among the respondents, regardless of sex, age and country, notwithstanding the fact that the respondent's knowledge about intimate partner violence might have been distorted before participating in the survey.

The insignificant differences in the results for the traits analyzed in this study demonstrate the predominance of traditional masculinity and femininity patterns in the mindset across genders and cultures. This suggests that the perpetuation of abusive behavior, whether by inflicting it or allowing it to happen may be contributing to the lack of visibility and cultural normalization of intimate partner violence.

Psychological abuse was found to be the predominant type of violence (94.8 percent in Santo Domingo and 76.2 percent in Tijuana) upon completion of the last part of the questionnaire, regardless of gender in both countries. The next type of violence in order of importance was physical violence (especially in the case of Santo Domingo), also showing no differences between men and women.

Dasgupta (2002) claims that the number of women who exert physical violence against their partners is comparable to that of men in terms of context, motivation, results and consequences. However, it is also true, as indicated by Alegría and Rodríguez (2015) that physical violence perpetrated by females is less likely to result in injury. Therefore, when discussing bi-directionality of violence, it is important to consider that women, as compared to men, are much more likely to suffer a serious injury during physical altercations (Archer, 2000; Chiodo, Crooks, Wolfe, McIsaac, Hughes \& Jaffe, 2011; Dasgupta, 2002; Desmarais, Reeves, Nicholls, Telford \& Fiebert, 2012; Muñoz et al. 2007). The prevalence of female deaths perpetrated by their male companions has become a matter of great social concern in recent years, as shown by current research (Adam, 2013). Alegría and Rodriguez (2015) who also emphasized that when women caused serious injury to men, it was usually in self-defense (see also Straus, Gelles, \& Steinmetz, 1980; Echeburúa, 2003; Miller \& White, 2003; Hernández, 2014). Although this study did not measure the frequency of abusive behavior (a deficiency in the questionnaire which would have provided further insights), research which has considered this variable (the 
case of UCM, 2013) showed a higher percentage of repeated abuse by men toward women.

In both countries, the principal causes that unleashed violent episodes cited by the survey respondents were jealousy and insecurity, followed by the influence of education imbued with patriarchal cultural values, (machismo), poor communication, and finally, financial hardship.

The predominance of these four fundamental causes presents some variations by country and gender. While men in both Tijuana and Santo Domingo primarily identified their jealousy and insecurities as the causes of their violent manifestations, women in Santo Domingo pointed at cultural and educational elements along with machismo as the principal causes of the abuse they received. In contrast, women from Tijuana highlighted jealousy (and a lack of trust by their partner) as the primary motivator of violence. Ultimately, all of these assumed causes are associated with insecurity and low self-esteem, of which the former is not only associated with internalized patriarchal patterns, but also with a sense of uncertainty about the relationship. Economic hardship and poor communication occupy the last places in the order of predominance but are nevertheless present and associated with the men's image as the principal provider.

This study, groundbreaking, among other things, for studying the abuse from women against men in the Dominican Republic, demonstrates its relevance and importance in the sense that it has affected in many aspects the general idea about gender violence which is believed to be only exerted by men against women, which constitutes a barrier to undertake new studies in this bidirectional way, and that in turn we break with the rigid gender roles, and identify other types of violence that, although no of the physical type, in the long run could result in dramatic consequences.

To deepen this kind of studies, we could consider the inclusion of a "frequency" variable in regards to abusive conducts so that it may be further measured and analyzed (as in the case of UCM, 2013), that would enrich this type of research.

Finally, it should also be noted that taking in to account the results obtained by this research project, there are already prevention programs for both sexes scheduled to be undertaken at the Universidad de la Tercera Edad (UTE) in Santo Domingo. 


\section{Notes}

1. The Adult as an Object and Subject of Violence; a collaborative effort between Universidad de la Tercera Edad (UTE) de Santo Domingo in the Dominican Republic and El Colegio de la Frontera Norte (El Colef), 2003-2018.

2. This study has rigorously followed the Council for International Organizations of Medical Sciences International ethical guidelines for health-related research involving humans (CIOMS, 2016), especially Nos. 5 and 6.

3. Where physical violence is characterized by physical acts of abuse, such as punching, kicking, slapping, pushing, biting and strangulation attempts, the psychological type includes any event that diminishes the integrity of the person, such as verbal attacks, power and control actions such as the isolation from family and friends, persecution and harassment, verbal threats, degradation and blackmail, sexual violence involves forced sex actions (genital, anal or oral from the woman towards the aggressor or the aggressor towards women, objects inserted in vagina or anus), sex in public, physical violence during intercourse, threats or degradation for rejecting sex, and forced use of pornographic films. And the economic happens when the couple takes away the money they earn, is prevented from spending it for their benefit or that of their family, its use is controlled or denied to diminish their independence.

4. Being a victim of violence or perpetuating a specific type of violence against another person does not exclude him or her from being a victim of, or perpetuating, other types of violence by or towards that same person.

5. In both countries, the participation in the study was completely voluntary, that is, once the application of the anonymous survey was agreed upon with the universities, all students who wished to participate did so once they expressed their informed consent.

6. Instances when, with slight differences, those respondents belonging to the age group from 41 to 63 years of age showed higher percentages, followed by age groups between 31 to 40 years.

7. Excluding the article by Rosales et al. (2017), which is an extension of this same study.

\section{References}

Abelino Ferrer, L., \& Monroy Juárez C. (2016). "Dime cuánto sufres y te diré cuánto amas." Un estudio exploratorio sobre violencia en el noviazgo con jóvenes universitarios de la FES Iztacala. Revista Electrónica de Psicología de Iztacala, 19(2), 1131-1152. Retrieved from http://www.journals.unam.mx/index.php/repi/article/view/57277 
Adam, A. (2013). Una revisión sobre violencia de género. Todo un género de duda. Gaceta Internacional de Ciencias Forenses, 9, 23-31 . Retrieved from https://dialnet.unirioja.es/servlet/articulo?codigo=4456934

Alegría del Ángel, M., \& Rodríguez, A. (2015). Violencia en el noviazgo: perpetración, victimización y violencia mutua. Una revisión. Actualidades en Psicología, 29(128), 57-72. Retrieved from https://revistas.ucr.ac.cr/index.php/actualidades/article/view/16008

Amor, P., Echeburúa, E., De Corral, P., Zubizarreta, I., \& Sarasua, B. (2002). Repercusiones psicopatológicas de la violencia doméstica en la mujer en función de las circunstancias del maltrato. International Journal of Clinical and Health Psychology, 2(2), 227-246 Retrieved from http://www.aepc.es/ijchp/articulos_pdf/ijchp-38.pdf

Amórtegui-Osorio, D. (2005). Violencia en el Ámbito Universitario: El caso de la Universidad Nacional de Colombia. Revista de Salud pública, 7(2), 157-165.

Retrieved

from

https://www.scielosp.org/article/ssm/content/raw/?resource_ssm_path=/

media/assets/rsap/v7n2/v7n2a04.pdf

Aparecida, M., da Cruz, M., Coelho, M., Fiqueira, C., \& Carvalho, M. (2016). Prevalência e características da violência no namoro entre adolescentes escolares de Portugal. Escola Anna Nery, 20(1), 183-191. Retrieved from http://www.scielo.br/pdf/ean/v20n1/1414-8145-ean-2001-0183.pdf

Archer, J. (2002). Sex Differences in Physically Aggressive Acts Between Heterosexual Partners. A Meta-Analytic Review. Aggression and Violent Behavior, 7, 313-351. Retrieved from https://pdfs.semanticscholar.org/b3e2/8e5fddd737d30a714da02ec8ab585 5d902f0.pdf

Argoff, M.C. (2009). La abierta competencia entre el reconocimiento jurídico y la valoración social. El caso de la violencia de pareja. Civitas. Revista de Ciencias Sociales, 9(3), 402-417. Retrieved from http://revistaseletronicas.pucrs.br/ojs/index.php/civitas/article/view/6899

Bogaert García, H. (2014). La violencia conyugal en la República Dominicana. Ciencia y Sociedad,39(4), 573-600. From: https://repositoriobiblioteca.intec.edu.do/bitstream/handle/123456789/15 80/CISO20143904-573-600.pdf 
Cáceres, J. (2007). Violencia doméstica: lo que revela la investigación básica con parejas. Papeles del Psicólogo, 28(1), 104-115. Retrieved from http://www.redalyc.org/pdf/778/77828108.pdf

Castro-Borunda, Z.; Naranjo-Cantabrana, M., \& Ayala-Bobadilla, N. (2015). La violencia en los jóvenes de nuevo ingreso del ITLM. $R a$ Ximhai, 11(4), 55-74. Retrieved from http://www.redalyc.org/pdf/461/46142596003.pdf

Chávez, J., \& Rodríguez L. (2015). Percepción de la violencia de género en las y los jóvenes universitarios. Trabajo Social Sin Fronteras, 63-74. Retrieved

from http://biblos.ucol.mx/bitstream/123456789/252/3/SF13008.pdf

Chiodo, D., Crooks, C., Wolfe, D., McIsaac, C., Hughes, R., \& Jaffe, P. (2011). Longitudinal Prediction and Concurrent Functioning of Adolescent Girls Demonstrating Various Profiles of Dating Violence and Victimization. Prevention Science, 13(4), 350-359. Retrieved from http://journals.sagepub.com/doi/abs/10.1177/0886260514564070?journal Code $=$ jiva

Council for International Organizations of Medical Sciences (CIOMS). (2016). International Ethical Guidelines for Health-Related Research Involving Humans. Geneva: Council for International Organizations of Medical Sciences. Retrieved from https://cioms.ch/wpcontent/uploads/2017/01/WEB-CIOMS-EthicalGuidelines.pdf

Cubillas, M., Valdez, E., Domínguez, S., Román, R., Hernández, A., \& Zapata, J. (2016). Creencias sobre estereotipos de género de jóvenes universitarios del norte de México. Perspectivas en Psicología, 12(2), 217-230.

Retrieved

from http://www.redalyc.org/pdf/679/67946836004.pdf

Cucco, M. (2010). Hombres y mujeres ¿Sólo un problema de rosa y azul? La formación del sujeto que somos. Capitalismo, relaciones sociales y vida cotidiana. Nuestra Ciencia. Revista del Colegio de Psicólogos de la Provincia de Córdoba, Argentina, 14, 37-46. Retrieved from http://revsexologiaysociedad.sld.cu/index.php/sexologiaysociedad/article/ view/194/279

Dasgupta, D. S. (2002). A Framework for Understanding Women's Use of Nonlethal Violence in Intimate Heterosexual Relationships. Violence Against Women, 8(11), 1364-1389. doi: 10.1177/107780102237408 
Desmarais, S.; Reeves, K.; Nicholls, T.; Telford, R., \& Fiebert, M. (2012). Prevalence of Physical Violence in Intimate Relationships, Part 2: Rates of Male and Female Perpetration. Partner Abuse, 3(2), 170-198. doi: http://dx.doi.org/10.1891/1946-6560.3.2.140

Echeburúa, E. (2003). Violència en la parella: claus psicològiques. Fòrum. Revista del Centre d'Estudis Juridics, 2, 12-23. Retrieved from https://www.uv.es/GICF/4A1_Adam_GICF_09.pdf

El Colegio de la Frontera Norte (El Colef). (2006). Encuesta de Salud Reproductiva en la Adolescencia de Baja California (ENSARABAC). Unpublished database. Tijuana, México: El Colegio de la Frontera Norte/ Consejo Nacional de Población. Retrieved from https://www.colef.mx/emif/resultados/publicaciones/publicacionesnte/pu bnte/EMIF\%20NORTE\%202010.pdf

Escudero Nafs, A., Polo Usaola, C., López Gironés, M., \& Aguilar Redo, L. (2005). La persuasión coercitiva, modelo explicativo del mantenimiento de las mujeres en una situación de violencia de género. I: Las estrategias de la violencia. Revista de la Asociación Española de Neuropsiquiatría, (95), 85-117. Retrieved from http://scielo.isciii.es/scielo.php?script=sci_arttext\&pid=S0211$57352005000300006 \& \operatorname{lng}=$ es \& tlng=es

Fernández, T. (2014). La educación sexual y de género vs. el maltrato en la pareja. Escenario sobre la violencia en jóvenes de Baja California. Estudios Fronterizos, 15(30), 73-96. Retrieved from http://www.scielo.org.mx/scielo.php?script=sci_arttext\&pid=S018769612014000200003

Fernández, T., Martínez, F., Unzueta, C., \& Rojas, E. (2016). Violencia hacia los varones entre parejas jóvenes universitarias de Tijuana, México. Enseñanza e Investigación en Psicología, 21(3), 255-263. Retrieved from http://www.redalyc.org/pdf/292/29248182005.pdf

Fernández, T. \& González, H. (2018). Violencia en parejas universitarias. Consideraciones socioculturales. Enseñanza e Investigación en Psicología, 23(3), 318-330. Retrieved from http://www.redalyc.org/pdf/805/80533065002.pdf

Fernández, T. \& Pérez, R. (2007). Autoestima y violencia conyugal: un estudio realizado en Baja California. México: El Colegio de la Frontera 
Norte/Miguel Ángel Porrúa Retrieved from https://libreria.colef.mx/detalle.aspx?id=7434

Fernández, T., Pérez Abreu, R., \& Anguiano, M. (2000). Autoestima, antigüedad laboral e ingreso salarial: Su influencia en mujeres y hombres. Revista Enseñanza e Investigación en Psicología, 5(2), 319-332. Retrieved from http://www.academia.edu/1933412/Autoestima_antig\%C3\%BCedad_lab oral_e_ingreso_salarial_Su_influencia_en_mujeres_y_hombres_2000_

Fondo de Población de las Naciones Unidas - República Dominicana (UNFPA). (2017). Datos sobre violencia contra la mujer en República Dominicana. Santo Domingo, Dominican Republic: Fondo de Población de las Naciones Unidas. Retrieved from http://dominicanrepublic.unfpa.org/sites/default/files/pubpdf/datos_violencia_UNFPA_300316\%281\%29.pdf

Fonseca, C., \& Quintero, M. L. (2008). Igualdad de género entre jóvenes universitarios de la Universidad Autónoma del Estado de Hidalgo en la ciudad de Pachuca. In C. Fonseca, \& M. L. Quintero, coords., Temas emergentes en los estudios de género (pp. 173-183). México: Miguel Ángel Porrúa.

Formental Hernández, S., Hernández Pita, I., \& Fernández de Juan, T. (2014). Masculinities in Cuba: Description and Analysis of a Case Study from a Gender Perspective. Masculinities and Social Change, 3(3), 220247. doi: 10.4471/MCS.2014.53

García, M., Pico, M., Sánchez, S., Savall, F., Celda, N., Blasco, C., \& Martínez, M. (2005). Assessing physical, sexual, and psychological violence perpetrated by intimate male partners toward women: a Spanish cross-sectional study. Violence and Victims, 20, 99-123. Retrieved from http://psycnet.apa.org/record/2005-08490-007

Glass, N., Fredland, N., Campbell, J., Yonas, M., Sharps, P., \& Kub, J. (2003). Adolescent Dating Violence: Prevalence, Risk Factors, Health Outcomes, and Implications for Clinical Practice. Journal of Obstetric, Gynecologic, \& Neonatal Nursing, 32(2), 227-238. Retrieved from https://jhu.pure.elsevier.com/en/publications/adolescent-dating-violenceprevalence-risk-factors-health-outcome-3

Goinheix'S, S. (2012). Notas sobre la violencia de género desde la sociología del cuerpo y las emociones. Revista Latinoamericana de 
Estudios Sobre Cuerpos, Emociones y Sociedad, 8, 43-54. Retrieved from https://www.google.pt/url?sa=t\&rct=j\&q=\&esrc=s\&source=web\&cd=1\& ved=2ahUKEwi4l_2xuYbeAhXKslkKHWelA9kQFjAAegQICRAC\&url =https $\% 3 \mathrm{~A} \% 2 \mathrm{~F} \% 2 \mathrm{Fdialnet}$.unirioja.es\%2Fdescarga $\% 2$ Farticulo\%2F397 1045.pdf\&usg=AOvVaw1Mt7XsJpF9MpYQpec4828V

González, H. \& Fernández, T. (2014). Hombres violentados en la pareja. Jóvenes de Baja California, México. Culturales, 2(2), 129-155. Retrieved from http://www.redalyc.org/pdf/694/69432742006.pdf

Hernández, A. (2007). La participación de las mujeres en las interacciones violentas con su pareja: Una perspectiva sistémica. Enseñanza $e$ Investigación en Psicología, 12(2), 315-326. Retrieved from http://www.redalyc.org/pdf/292/29212207.pdf

Hernández, I. (2014). Violencia de Género. Una mirada desde la sociología. Havana: Editorial Científico Técnica. Retrieved from http://www.ts.ucr.ac.cr/binarios/libros/libros-000059.pdf

Karakurt, G., \& Cumbie, T. (2012). The relationship between egalitarianism, dominance and violence in intimate relationships. Journal of Family Violence, 27(2), 115-122. Retrieved from https://cwru.pure.elsevier.com/en/publications/the-relationship-betweenegalitarianism-dominance-and-violence-in-2

Kaukinen, C., Gover, A. R., \& Hartman, J. L. (2012). College women's experiences of dating violence in casual and exclusive relationships. American Journal of Criminal Justice, 37(2), 146-162. Retrieved from http://psycnet.apa.org/record/2012-11786-002

Martínez, J., Vargas, R., \& Novoa, M. (2016). Relación entre la violencia en el noviazgo y observación de modelos parentales de maltrato. Psychologia. Avances de la Disciplina. 10(1), 101-112. Retrieved from http://www.redalyc.org/pdf/2972/297245905010.pdf

McDonell, J., Ott, J., \& Mitchell, M. (2010). Predicting dating violence victimization and perpetration among middle and high school students in a rural southern community. Children and Youth Services Review, 32(10), 1458-1463 Retrieved from http://psycnet.apa.org/record/201017145-018

Mejía, M. (November 26 2011). Violencia económica contra la mujer. Diario Libre. Santo Domingo, Dominican Republic. 
https://www.diariolibre.com/noticias/violencia-econmica-contra-lamujer-GKDL314604

Méndez, A. C. (2012). Jóvenes sufren violencia en el noviazgo en un 30\%: Imjuve. Milenio Diario. Retrieved from http://anuario.upn.mx/2012/index.php/milenio-diario/68898-jovenessufren-violencia-en-el-noviazgo-en-un-30imjuve.html?tmpl=component\&print=1\&page

Miller, J., \& White, N. (2003). Gender and Adolescent Relationship Violence: A Contextual Examination. Criminology, 41(4), 1207-1248 Retrieved from https://onlinelibrary.wiley.com/doi/abs/10.1111/j.17459125.2003.tb01018.x

Mohamed, L., Herrera, L., \& Carracedo, S. (2014). Violencia de pareja en jóvenes estudiantes universitarios de diferente origen cultural. Dedica. Revista de Educação e Humanidades, 5, 223-236. Retrieved from https://www.researchgate.net/publication/275329503_Violencia_de_parej a_en_jovenes_estudiantes_universitarios_de_diferente_origen_cultural

Montesinos, R., \& Carrillo, R. (2011). El crisol de la violencia en las universidades públicas. $E l$ Cotidiano, 170, 49-56. Retrieved from http://www.redalyc.org/pdf/325/32520935006.pdf

Moral, J., \& López, F. (2012). Modelo recursivo de reacción violenta en parejas válido para ambos sexos. Boletín de Psicología, 105, 61-74. Retrieved from https://www.uv.es/seoane/boletin/previos/N105-4.pdf

Moral, J., \& López, F. (2013). Premisas socioculturales y violencia en la pareja: diferencias y semejanzas entre hombres y mujeres. Estudios sobre las Culturas Contemporáneas, 19(38), 47-71. Retrieved from http://www.redalyc.org/pdf/316/31629858004.pdf

Moreno, F. (1999). La violencia en la pareja. Revista Panamericana de Salud Pública, 5(4/5), 245-551. Retrieved from https://scielosp.org/article/rpsp/1999.v5n4-5/245-258/es/

Muñoz, M., Graña, L., O’Leary, K.D., \& González, P. (2007). Aggression in Adolescent Dating Relationships: Prevalence, Justification, and Health Consequences. Journal of Adolescent Health, 40(4), 298-304. Retrieved from https://www.jahonline.org/article/S1054-139X(06)00570-2/fulltext

Nocentini, A., Menesini, E., \& Pastorelli, C. (2010). Physical Dating Aggression Growth During Adolescence. Journal of Abnormal Child Psychology, 38(3), 353-365. doi: 10.1007/s10802-009-9371-8 
Olvera, J., Arias, J., \& Amador, R. (2012). Tipos de violencia en el noviazgo: estudiantes universitarias de la UAEM, Zumpango. Revista Electrónica de Psicología Iztacala, 15(1), 150-171. Retrieved from http://www.iztacala.unam.mx/carreras/psicologia/psiclin/vol15num1/Vol 15No1Art8.pdf

Pico, M., Echeburúa, E. \& Martínez, M. (2008). Personality disorder symptoms as a result of chronic intimate male partner violence. Journal of Family Violence, 23, 577-588. doi: 10.1007/s10896-008-9180-9

Ramírez, F. A. (2002). Violencia masculina en el hogar. Alternativas y soluciones. México: Pax México. Retrieved from http://www.redalyc.org/pdf/325/32511304.pdf

Ramírez Rivera, C., \& Núñez Luna, D. (2010). Violencia en la relación de noviazgo en jóvenes universitarios: un estudio exploratorio. Enseñanza e Investigación en Psicología,15(2), 273-283. Retrieved from http://www.redalyc.org/pdf/292/29215980003.pdf

Ramírez, I. L., \& Smithey, M. (2008). Intimate Partner Violence Victimization Among Mexican University Students: A Descriptive Study and Analysis of Demographic Factors. Contemporary Issues in Criminology and the Social Sciences, 2(4), 34-53. Retrieved from https://www.aacademica.org/dr.jose.luis.rojas.solis/10.pdf

Rangel, Y. Y., \& García, M. (2010). Influencia del rol de género en las formas de canalización de violencia en estudiantes universitarios mexicanos. Paper presented at the XI Congreso Virtual de Psiquiatría INTERPSIQUIS 2010. Retrieved from https://psiquiatria.com/bibliopsiquis/influencia-del-rol-de-genero-en-lasformas-de-canalizacion-de-violencia-en-estudiantes-universitariosmexicanos

Rocha Sánchez, T. E. (2009). Desarrollo de la identidad de género desde una perspectiva psico-socio-cultural: Un recorrido conceptual. Revista Interamericana de Psicología/Interamerican Journal of Psychology, 43(2), 250-259. Retrieved from http://www.redalyc.org/pdf/284/28412891006.pdf

Rodriguez, J. A. (2014). When Man Falls Provider. Masculinity, Unemployment and Psychological Distress in the Family. A methodology for the Search of Affective Normalization. Masculinities and Social Change, 3(2), 173-190. doi: 10.4471/MCS.2014.49 
Rodríguez, S. (2015). Violencia en parejas jóvenes: estudio preliminar sobre prevalencia y motivos. Pedagogía Social. Revista Interuniversitaria, 25, 251-275.

Retrieved

from

http://www.redalyc.org/pdf/1350/135043709011.pdf

Rojas-Solís, J. (2013). Violencia en el noviazgo de universitarios en México: Una Revisión. Revista Internacional de Psicología, 12(2). Retrieved from http://www.revistapsicologia.org/index.php/revista/article/view/71

Rojas Solís, J. L. (2016). Hombres maltratados. Masculinidad y control social. Autora: Laia Folguera Cots. [Review of the book]. Ra Ximhai, 12(4), 225-228. Retrieved from http://www.redalyc.org/pdf/461/46146927014.pdf

Rosales, M., Florez, L., \& Fernández, T. (2017). La violencia de pareja: análisis en una población universitaria de Santo Domingo. Enseñanza e Investigación en Psicología, 22(2), 174-182. Retrieved from http://www.redalyc.org/pdf/292/29255774004.pdf

Rosales M. \& Florez, L. (2016). Sobre la violencia en la pareja: Análisis en una población universitaria de Santo Domingo, República Dominicana (Disertation). Universidad UTE, Santo Domingo, Dominican Republic.

Ruiz, I., Plazaola, J. \& Del Río, M. (2007). Physical health consequences of intimate partner violence in Spanish women. European Journal of Public Health, 17, 437-443. doi:10.1177/0886260507301227

Shook, N., Gerrity, D., Jurich, J., \& Segrist, A. (2000). Courtship Violence Among College Students: A Comparison of Verbally and Physically Abusive Couples. Journal of Family Violence, 15(1), 1-22.

Soldevila, M. (November 15 2011). El agresor común no parece un monstruo. El Listín. Santo Domingo, Dominican Republic. Retrieved from https://www.listindiario.com/la-republica/2011/11/25/212299/elagresor-comun-no-parece-un-monstruo

Soriano, A. (2011). La violencia en las relaciones de pareja en estudiantes universitarios. Propuestas educativas. Pedagogía Social, 18, 87-97. Retrieved from http://www.redalyc.org/pdf/1350/135022618007.pdf

Sosa, I., \& Menkes, C. (2016). Amarte duele. La violación sexual en las relaciones de noviazgo. Un análisis de sus determinantes sociales. Papeles de Población, 22(87), 43-62. Retrieved from http://www.redalyc.org/articulo.oa?id=11244805003 
Straus M. A., Gelles, R. J., \& Steinmetz, S. K. (1980). Behind Closed Doors: Violence in the American Family. Garden City, New York: Anchor Press/ Doubleday. Retrieved from https://www.amazon.es/Behind-Closed-Doors-ViolenceAmerican/dp/1412805910

Swahn, M., Alemdar, M., \& Whitaker, D. (2010). Nonreciprocal and Reciprocal Dating Violence and Injury Occurrenceamong Urban Youth. Western Journal of Emergency Medicine, 11(3), 264-268. Retrieved from https://www.ncbi.nlm.nih.gov/pmc/articles/PMC2941364/

Trujano, P., Martínez, A. E., \& Camacho, S. I. (2010). Varones víctimas de violencia doméstica: Un estudio exploratorio acerca de su percepción y aceptación. Diversitas. Perspectivas en Psicología, 6(2), 339-354. Retrieved from http://www.scielo.org.co/pdf/dpp/v6n2/v6n2a10.pdf

Tubert, S. (ed.) (2003). Del sexo al género. Los equívocos de un concepto. Valencia, Spain: Editorial Cátedra-Universidad de Valencia. Retrieved from https://dialnet.unirioja.es/servlet/libro?codigo $=3015$

Universidad Complutense de Madrid (UCM). (2013). La juventud universitaria ante la igualdad y la violencia de género. Madrid, Spain: Universidad Complutense de Madrid/ Ministerio de Sanidad, Servicios Sociales e Igualdad. Retrieved from http://www.upm.es/sfs/Rectorado/Gerencia/Igualdad/Documentos/Juvent ud_Universitaria_ante_igualdad_y_violencia_de_genero_\%202012.pdf

Vélez, L. (2011). Representaciones y estereotipos femeninos en los medios de comunicación. Paper presentad at the XII Encuentro Internacional de Centros de Cultura. Universidad Popular Autónoma del Estado de Puebla. Puebla, México. Retrieved from http://www.upaep.mx/micrositios/12encuentro/Ponencia\%20Lilia\%20Ve lez.pdf

Zamudio, F., Ayala, M., \& Andrade, M. (2011). Violencia en el alumnado de la Universidad Autónoma Chapingo, según género. Paper presented at the XI Congreso Nacional de Investigación Educativa. Convivencia, Disciplina y Violencia en las Escuelas. Universidad Autónoma Chapingo. Texcoco, México. Retrieved from http://www.comie.org.mx/congreso/memoriaelectronica/v11/docs/area_1 7/1443.pdf 
Zamudio, M. (2014). El proceso de revisión de la violencia en un grupo de varones. In J. Figueroa \& A. Salguero, coords., Y si hablas de...sde tu ser hombre?: Violencia, paternidad, homoerotismo y envejecimiento en la experiencia de algunos varones (pp. 115-164). México: El Colegio de México-Centro de Estudios Demográficos, Urbanos y Ambientales. Retrieved from https://estudiosdemograficosyurbanos.colmex.mx/index.php/edu/article/v iew/1499/1728

Teresa Fernández de Juan, is PhD. in Psychological Sciences and Profesor Researcher at the Cultural Studies Departament at El Colegio de la Frontera Norte in Tijuana, Mexico.

Lourdes Florez Madan has a Masters of Sciences in Human Sexuality, and is currently Director of the Research Department at the Universidad de la Tercera Edad (UTE) in Santo Domingo, Dominican Republic.

Contact Address: Direct correspondence to Teresa Fernándes de Juan, Escénica Tijuana-Ensenada, Km 18.5, San Antonio del Mar, 22560 Tijuana, Mexico,email: teresaf@colef.mx 
Instructions for authors, subscriptions and further details:

\section{http://mcs.hipatiapress.com}

\section{The Social Life of Gender}

Vladia Ionescu ${ }^{1}$

1) Universidad de Barcelona, Spain

Date of publication: October $21^{\text {th }}, 2018$

Edition period: October 2018-February 2019

To cite this article: Ionescu, V. (2018). The Social Life of Gender. [Review of the book]. Masculinities and Social Change 7(3), 313-314. doi:

10.17583/MCS.2018.3806

To link this article: http://dx.doi.org/10.4471/MCS.2018.3806

\section{PLEASE SCROLL DOWN FOR ARTICLE}

The terms and conditions of use are related to the Open Journal System and to Creative Commons Attribution License (CC-BY). 

MCS - Masculinities and Social Change Vol. 7 No. 3 October 2018

pp. 313-314

\section{Reviews (I)}

Ray, R., Carlson, J., \& Andrews, A. (Eds.). (2017). The Social Life of Gender. New York: Sage Publication

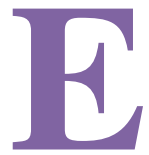

stamos ante un libro que presenta un análisis profundo sobre la masculinidad en dos países con tradición en este ámbito de estudio: Estados Unidos y Reino Unido. Además, la obra enfrenta dos paradigmas en los que se han movido las ciencias sociales y en especial, los men's studies en la era contemporánea: "la corporalidad" y "los sistemas". De esta forma se plantea la construcción social del género y su impacto en la estructura social, pero también se tiene en cuenta el efecto en las identidades y cuerpos de los sujetos.

En el libro hay diferentes capítulos donde se aborda el estudio de la masculinidad en Estados Unidos, haciendo hincapié, por ejemplo, en la figura del cowboy que tanto peso tiene en la socialización de género en este país. En otro capítulo también se aborda el influjo que tiene el ejército y el sector militar en la construcción de la identidad masculina. En este sentido, existe un capítulo donde se analiza el efecto que tuvieron los atentados del 11 de Septiembre del 2001, aprovechando algunas representaciones como la del libro Saturday de Ian McEwans y la película World Trade Center del director Oliver Stone. De hecho, como ya han hecho otras obras alrededor de los estudios de masculinidad, el libro incluye otros capítulos donde también se exploran las representaciones de artículos como el best seller The Dogs of War donde se presenta el heroismo a las "masculinidades mercenarias".

En el libro se estudian otras representaciones de la masculinidad que aparecen en diferentes libros, series y películas. En este sentido, resulta interesante el análisis que se efectúa sobre la serie de HBO Deadwood, la obra de William Gaddis o las representaciones cinematográficas sobre masculinidad, en películas recientes, que abordan la realidad de Wall Street.

2018 Hipatia Press

ISSN: 2014-3605

DOI: $10.17583 / \mathrm{MCS} .2018 .3806$ 


\section{Ionescu-The Social Life of Gender [Book Review]}

Resulta interesante también la incorporación de una pieza de Michael Kimmel, autor de referencia en el ámbito de las masculinidades, que habla sobre la supremacia blanca - y del hombre blanco en particular- en los Estados Unidos.

En definitiva, el libro nos aproxima a investigaciones recientes en el campo de las masculinidades que nos ilustran diferentes ejemplos de representaciones contemporáneas alrededor de la masculinidad. También nos enseña la existencia de estereotipos y disciminaciones que no podemos olvidar y que continúan perpetuando el esquema patriarcal de hombre blanco y heterosexual como representación hegemónica. Así, los autores y autoras de esta obra plantean unas líneas de trabajo futuras más que interesantes para seguir avanzando en la literatura científica de los men's studies.

Vladia Ionescu, Universidad de Barcelona. vladia.ionescu@gmail.com 


\section{Hipatia Press}

Instructions for authors, subscriptions and further details:

Hacerse Hombres. La Construcción de Masculinidades desde las Subjetividades

Liviu Catalin Mara ${ }^{1}$

1) Universitat Rovira i Virgili, Spain

Date of publication: October $21^{\text {th }}, 2018$

Edition period: October 2018-February 2019

To cite this article: Mara, L.C. (2018). Hacerse Hombres. La Construcción de Masculinidades desde las Subjetividades. [Review of the book].

Masculinities and Social Change 7(3), 315-317. doi:

10.17583/MCS.2018.3819

To link this article: http://dx.doi.org/10.4471/MCS.2018.3819

PLEASE SCROLL DOWN FOR ARTICLE

The terms and conditions of use are related to the Open Journal System and to Creative Commons Attribution License (CC-BY). 
MCS - Masculinities and Social Change Vol. 7 No. 3 October 2018

pp. 315-317

\section{Reviews (II)}

Muñoz Sanchez, H. (2017). Hacerse hombres. La construcción de masculinidades desde las subjetividades. Medellín: Universidad de Antioquia

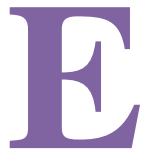

1 libro "Hacerse hombres. La construcción de masculinidades desde las subjetividades" es el resultado de una investigación doctoral, y, por tanto, se enmarca dentro de un unos objetivos y metodología científicos. Así, el objetivo de esta investigación era analizar el proceso de construcción y significación de los hombres limitándose a relatos de vida de hombres heterosexuales que se identifican como varones, en Medellín (Colombia). No obstante, el autor subraya la existencia de múltiples tipos de masculinidades, como también la importancia de elementos socio-economicos y culturales y también la interseccionalidad con sistemas de opresión que contribuyen a la construcción del género, tanto masculino cómo femenino.

El punto de partida de este libro es justamente que tanto los hombres cómo las mujeres son sujetos generizados, aunque a nivel de políticas este hecho no se ha tomado en cuenta. El autor destaca que en la elaboración de dichas políticas se ha tomado en cuenta el "sexo" y no el "género", y esto ha implicado ignorar y negar el papel y la cción de los varones en la realidad social que se pretendía resolver en primera instancia. Por tanto, ha quitado la responsabilidad que corresponde a los varones en la tarea de alcanzar la igualdad entre hombres y mujeres, que justifica el fracaso de estos planes. Por eso, continúa el autor, habría que introducir también a los hombres en esta lucha.

Otra implicación es que cómo la construcción del género es un fenómeno social, en el que intervienen muchos actores y dispositivos sociales, esta desigualdad no existe únicamente entre hombres y mujeres sino también en

2018 Hipatia Press

ISSN: 2014-3605

DOI: $10.17583 / \mathrm{MCS} .2018 .3819$ 


\section{Mara-Hacerse Hombres [Book Review]}

las relaciones intragénero. Según el autor, en el caso de los varones hay un tipo de masculinidad dominante que oprime a las demás masculinidades, pero esteas relaciones de poder son mitigadas por la promesa de los privilegios que vienen asociados al modelo del varón heterosexual blanco de clase media. En este punto del argumento, el autor identifica la posibilidad y la importancia de la investigación académica para explicar y hacer visibles estas fisuras y de las políticas para crear un proyecto colectivo que lleve a la liberación de la opresión y de los altos costes emocionales y sociales que suponen para los individuos.

En cuanto a su investigación en Medellín, la contribución de este libro es resaltar los tres elementos definitorios del modelo de hombre: la autonomía económica, la autoridad de un núcleo familiar y una heterosexualidad activa. El primero está vinculado al nivel de ingresos y a las pertinencias que pueda acumular, lo cual llevará a más o a menos éxito con las mujeres. El segundo elemento tiene que ver con formar una familia propia dónde ejercer esa autoridad o poder. El tercer elemento tiene que ver con la práctica activa y exhibición pública de la heterosexualidad. Para conseguir estos tres elementos los hombres tienen varias pautas o normas a seguir (por ejemplo la diferenciación de lo femenino; uso de la violencia, o la prohibición de la homosexualidad), que a su vez estan reforzadas y vigiladas por los grupos de iguales, amigos y/o las familias.

A nivel práctico, esta construcción de los hombres que es similar en otras partes del mundo, se ha visto puesta en duda y contestada desde los movimientos sociales, academia y las políticas publicas. El autor identificó en el estudio laincoherencia entre los discursos a favor de la igualdad que presentaban sus entrevistados con elementos de justificación de la diferenciación, dadas por el cuerpo diferente de los hombres y de las mujeres. Por lo tanto los jóvenes se enfrentan al dilema surgido de la existencia de una cultura machista y reforzada con elementos de lacultura narcoparamilitar y el paradigma de cambio a nivel internacional que aboga por la transformación hacia la igualdad entre hombres y mujeres.

Una contribución muy importante de este libro es la apuesta por la aproximación hacia el tema de la construcción social de los hombres desde las subjetividades de los hombres que participaron en el estudio. Este hecho ha facilitado en los participantes en la investigación una reflexión y resignificación de los elementos y procesos sociales que les ha convertido en 


\section{MCS - Masculinities and Social Change, 7(3) 317}

los hombres que son. Una construcción que no es natural, como ellos lo tenían asumido, sino que es un proceso contingente, y que ellos mismos son unos sujetos generizados y que, por lo tanto, la lucha por la igualdad les concierne también a ellos. Finalmente el autor postula que 1 implicación de los hombres se tiene que tratar desde un enfoque colectivo, implicando a movimientos sociales y al Estado en esta lucha, para que la igualdad se pueda conseguir.

Liviu Catalin Mara, Universitat Rovira i Virgili. liviucatalin.mara@urv.cat 